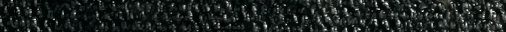

40,400 Whandili in

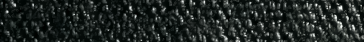

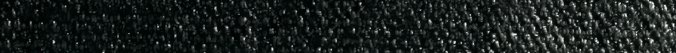

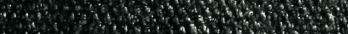

a.t.

(2)

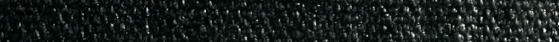

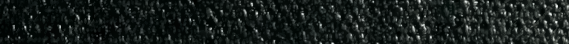
$\because 4 \times 2 \times 2 \times 2 \times 2$

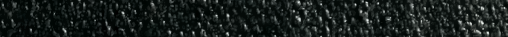

40 a $4, \quad x^{2}$ $\therefore \quad \therefore \quad \ldots$

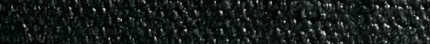
13. 2040

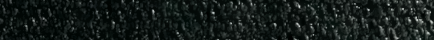

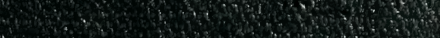

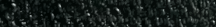
- 34003

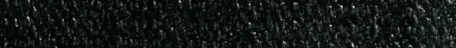

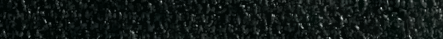

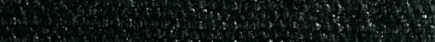
$\therefore \quad \therefore \quad+\cdots, y$

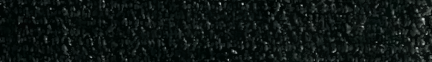
(1)

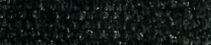

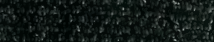

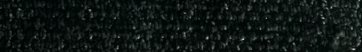
antenton

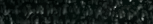

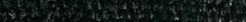

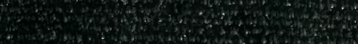
(1) 







\section{H. CHRIST. FABRICH}

HISTOR. NAT. OECON. ET CAMERAL. PROF. CHILONIENS. ACAD. HAFNIENS. NORWEG. ET BE. ROLINENS. SOC.
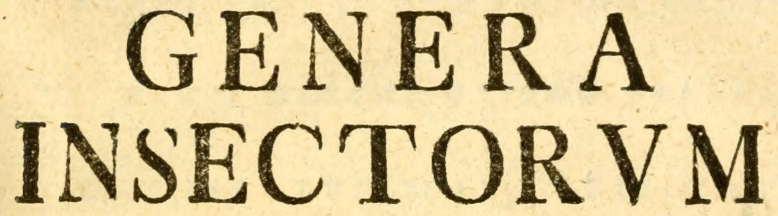

EORVMQVE

CHARACTERES NATVRALES SECVNDVM

NVMERVM, FIGVRAM,

SITVM ET PROPORTIONEM

OMNIVM PARTIVM ORIS

A DIECTA

MANTISSA SPECIERVM

NVPER DETECTARVM.

C H I L O N I I

LITTERIS MICH. FRIEDR. BARTSCHII.

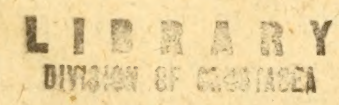


I gnorato genere proprio, nulla defcriptio, quamuis accurate tradita, certum demonfrat, red plerumque fallit. Carfalpin. 


\title{
PRINCIPI SERENISSIMO
}
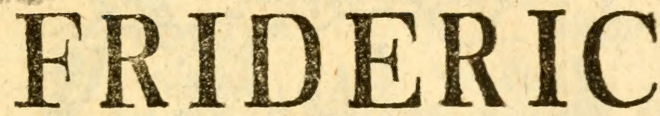

REGIO DANIAE NORWEGIAE HEREDI,

REGIS OPTIMI OPTIMO FRATRI,

SALVTIS PVPLICAE, REIIGIONIS,

\author{
A R T IVM
}

SCIENTIARVMRVE FAVTORI

ATQVE STATORI.

D. D. D.

KILIA E

CVLTOR DEVOTISSIMVS

I. C. FABRICIVS. 



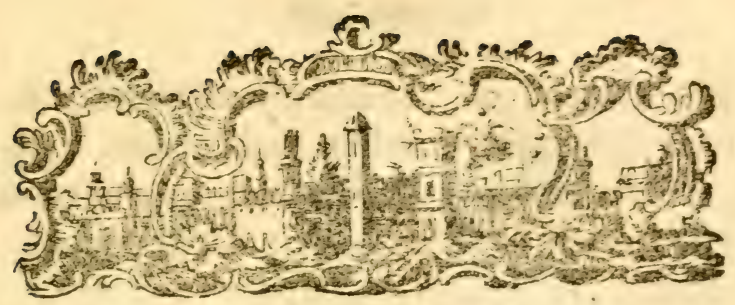

\section{PROLEGOMENA. .}

\section{(1)}

y/tema primum hiforiae naturalis continet fundamentum \& hoc fublato tota ruit fpecierum notitia, numero ipfo naturalium oppreffa. Me non fugit multos huius faeculi fcriptores fyftema fpernere leuioris fcientiae amatores, at foliditatem \& certitudinem omnino amittunt. Impofibile fane erit tantum fpecierum numerum, tot fizuras, tot defcriptiones fpecificas, quae in dies augentur, in memo. riam reuocare, nifi in ordinem redigantur. Mammalia quidem maiora, pauciora facilius diftinguuntur, at plantae $\&$ in fecta fere innumera, ipfo fyfemate adiuuante, difficilius rite determinantur \& absque fyftemate confufum omnino chaos praeberent. Omnes ideo hiftoriae natu- 


\section{*}

ralis heroes, omnes fcientiae felices cultores fyftemate quodam infifterunt, reliqui vero empirici obferuationibus vagis a fyltematicis redigendis fcientiam augent.

Syfemata entomologiae pauciora numerantur, at plura adhuc deíderantur. Quo plura enim elaborantur, eo maiorem foliditatem \& certitudinem attingit fcientia. Sequens fyfematicus antecedentis humeris infiftens altius circumfpicit.

Characteres generam fyifematis continent effentiam \& fcientiae foliditatem praebent. In his ideo comfruendis fumma adhibenda diligentia, ne characteribus hifce vacillantibus accumulatis totum tandem ruat fyftema.

Claracteres generum notas communes diftinctarum fpecierum fiftunt \& a partibus diftinctis, determinatis defumendi. Quo contantiores funt hae partes ad difinctionem generum affumtae, eo maiorem certitudinem \& praeftantiam habebit Iyftema. In his anteceffores omnes aberrant. Ante fummum a Linné vix vllum 
genus fixum rite determinatum inuenimus, vt illi merito inuentionis glorian crica genera concedere debeamus. At immortalis ipfe a Linné, qui in generibus plantarum condendis regulas tam arcte confrixit, qui characteres geverum a folo fore defumenciostan firenue affeuera uit in Entomologia eosdem characteres vndequaque collevit. Antennae, pedes, Thorax, omnes animalculi partes hic in confiderationem veniunt, ommes characteres pracbint generieos. Quae quacfo fyftema planturum, fi mox a radice, mox a caule, mox a foliis aut fore characteres generum defumerentur. Eadem Fotomologiae eft ratio. Inde oriuntur tot confuliones, tot contradictiones charac?eris fpecifici cum generico, quae nunquam enitantur, nifi ab omni fere fpecie proprium conderetur genus. Perfpexit perbene fummus vir hunc fyltematis defectum, ideoque rarius noua genera condidit \& magis habitui quam characteri confidit.

Omnes Linnaci fuccefiores fundamentum fyfematis vacillans haud rite cognofceutes 


\section{अन स\%}

fcentes magis fyftemati adhaeferunt, genera multa partibus variis diftincta introduxerunt, quo ipfo determinatio difficilior \& denominatio incertior.

Nouiflimi ideo entomologi ad figuras refugiunt, fyftema relinquunt, fpernunt $\&$ folis figuris praeftantifimis infecta determinare ftudent. Gratiflmam fcientiam hoc ipfo in libri memorialis formam, memoria tantum retinendam, redigunt. Omnes hos libros euoluere, omnes figuras examinare ad denominationem vnius cuiusque infecti neceffe eft $\&$ tamen determinatio ob varietates, quae nunquam figuris ex primi poffunt, faepius incerta euadit. Dolent hanc fcienciae incertitudinem omnes hiftoriae naturalis amatores. Nomina enim fi pereunt, perit \& cognitio rerum.

Nouam tentaui viam \& inftrumenta cibaria ad determinanda genera introduxi. Praeftant ob vfum, quem praebent. Praefant ob numerum \& certitudinem characterum, quos offerunt. Praeftant denique ob affinitatem cum characteribus reliquo- 
rum animalium a dentibus defumtis. $\mathrm{Me}$ non fugit partes hafce oris faepius minutiffimas difficilius erui oculoque armaro diftinguendas effe, at hand mirum mihi videtur, quod infectorum characteres, quae ipfa nudo oculo vix confpicua, armato defcribendi fint. Inftumenta certe cibaria infectorum floribus plantarum habita ratione magnitudinis corporum inter fe multo funt maiora, \& charaderes e partibus oris Lucani Cerui facilius eruuntur quam e foribus maximae Quercus. Omnia, quae hic defcripf, genera microfcopio paruo fimpliciffino examinaui \& $x$ gratus iucundusque fuit labor.

Inftrumenta cibaria plures continent partes ad diftinctionem gencrun omnium detectorum \& ynquam detegendorum fufficientes. Inuenimus enim I Palpos duos, quatuor, fex corumque articulos, $2 \mathrm{Cly}-$ pei difcum, limbum. 3 Mandibulas transuerfales earumque dentes apicesque 4. Maxillas transuerfales earumque dentes, lacinias. 5 Galeam. 6 Labii numerum, diuifonem, difcum, limbum. 7.Linguam

$$
\text { ) } 5
$$




\section{- $2005 \%$}

fpiralem convolutam. 8. Rofri vaginam eiusque articulos. 9. Roftri feras, 10 . Hauftelli vaginam eiusque valuulas. I $\%$.Haufelli fetas. 12 Probofcidis fipitem. I3. Probofcidis capitulum bilabiatum. Omnes hae partes numero, figura, proportione, fitu, differunt \& charafteres generum conftantes praebent omnino fuffeicntes.

In paucis generibus a quibusdam partibus oris characteres defumferunt Linné, Scopoli, vâi in Antliatorum claffe, quare non in omnibus \& ab omnibus, quum genera $\&$ in eorum fyftematibus a partibus oris determinata reliquis multo funt certiora, multo funt naturaliora.

Anternas, quibus anteceftores omnes tanti faciunt, femper generum characteribus appofui. Minus tamen ad difinguenda genera valent, quan plerique efimant. Characteres praebent pauciores, rapius inconfantes, incertos. In eadem fpecie interdum differunt $\&$ quibusdam generibus antennae omnino defunt. Ad ordines clafium determinandas magis valere 


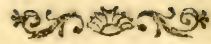

valere videntü quam ad generum determinationem.

Metamorphofts infectorum triplex in confruendis generibus femper confulenda. Nunquam quidem characterem proprium generum intrabit, attamen ad genera naturaliora viam monfrat. Infecta, quae metamorphofi difierunt, nunquam idem genus \& forte ne cundem quidem claffem intrabunt. Vnicum tantum exemplum Pulicis in contrarium habenus, attamen forte, fi plura infecta Pulici coniungenda detegerentur, propriam clafem conftituerent.

Layace \& Puppse ftructura nimis a fe inuicem differentes itidem vix vnquam fub eodem genere militabunt. Idem eft animal aetate tantum \& forma mutatum \& differens iuuentus differentem ínectutem indicare videtur. lure ideo Hydrophilum a Dytifcis aut Aeshnam \& Agrion a Libellulis feparaui. Differunt charactere, differunt \& fruciura laruae \& puppae. Netamorphof tamen \& fiructura laruac \& puppae magis adhaerere quam charactere abfur- 


\section{tecto}

abfurdum foret, quo ipfo primum fyftenatis rueret fundamentum.

Habitus Infectorum in confruendis generibus occulte obferuandus. Raro hic aberrabimus \& rariffime infecta habitu nimis a fe inuicem differentia, idem genus intrabunt. Genera naturalia funt \& talis differentiăm mifcela femper generum \& ryftematis defectum monfrat. Habitus tamen loco characteris nunquam affumendus $\&$ fpecies charactere fecundum affumta fytematis principia differentes nullo modo fub eodem genere collocandae. Vacillat alias fyftema \& tandem totum ruit aedificium proprio pondere generum incertorum oppreffum. Summus hiftoriae naturalis princeps ipfe a Linné hic paululum impegire mihi videtur in reliquis omnibus excellens. Naturae nimis adhaerens fyftematis filum ariadneum faepius amifit, vnde genera vacillant \& differentiae fpecificae character generico faepius contradicunt.

Icones nullas ad didi. Ad genera determinanda nullo modo valent. Multas

faepe 


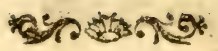

rape fpecies continent genera, quas omnes character genericus comprehendit. Characterem vero hunc icone exprimere omnino impoffibile, quum plures faepe fpecies eiusdem generis in quibusdam partibus oris a fe inuicem differunt, quamuis charactere generico conueniunt. Omnes hafce differentias eodem icone exprimere haud valemus. Confufionem ideo praeberent omnes icones ad generum determinationem confectas, quum femper quibusdam fpeciebus diffimiles effent.

Character genericus fecundum a Linné principia triplex inuenitur; effentialis nempe, artificialis \& naturalis.

EJjentialis character nota vnica, propria genus a reliquis omnibus diftinctum tradit. Character hic omnium optimus commendat fe breuitate, certitudine \& hoc dato genera facillime determinantur. Exempla huius characteris Syftema noAtum Entomologiae praebet vti in Melolontha, Lucano, Onifco, Scorpione, Oeftro aliisque; at in omnibus generibus 


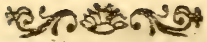

characterem obtinere effentialem vix ac ne vix quidem poffibile erit.

Artificialis character effentiali fubftituitur, vsque dum hic eruitur. Diftinguit gen snica nota aut pluribus a reliquis generibus fub eodem ordine militantibus. Nouo ideo detecto genere faepius mutandus, vt etiam ab hoc genere diftinguat. Charactere effentiali femper inferior, attamen vbi hic haud inuenitur, ille remper fuccedaneus adhibendus.

Natsialis charactir generum, quen hic trado, praebet notas omnes in inftrumentis cibariis onnibus fpeciebus eitusdem generis communes. Defcribit numerum, figuram, proportionem \& fitum omnium partium oris, quibus omnes fpecies fub eodem genere militantes conueniunt. Ofiert ideo hic character plurimas generis notas, continet characterem effentialem \& artificialem \& rite elaboratus certitudinem \& foliditatem ryftematis \& fcientiae efficit. Omnibus methodis adhuc conficiendis applicabilis erit, fi mo- 


\section{S.2.}

do isdem infrumentis cibariis fuperfrrantur.

Nomima generica antecefforum fancte femper feruaui. Verba valent praetio determinato \& characteres efientian, nomina tantum fonum generis continent. Confulionem praebet femper talis nominum mutatio \& nomine indeterminato, vago, vti pleraque funt, aliud nomen vagum fubfituere fuperuacaneum foret. Omne vero nomen genericum charactere proprio effentiali diftinguere hic labor, hoc opus. Plura vbi nomina eodem genere impofita funt, optimum vetufifimum retinendum.

Auctorum defcriptiones, figuras nunquam ad generum dererminarionem adhibere valui. Omnes oris partes plane omiferunt, ideoque quamuis figurae accurate traditae, tanen ad genera determinanda nullo modo valent.

Species infectorum in Syfemate Entomologiae Flensburgi 1775 dedi. Mantifam tantum fpecierum nuper detecta- 
rum hic addidi. Communicatae funt nouae hae fpecies partim a fautoribus, amicis Anglis \& Germanis, partim a commilitonibus Chilonienfibus amiciffmis, quibus omnibus me obitrictiffimum fateor.

Rationem \& leges fyftematis noftrae, fi fata velint, in philofophia entomologica dabo. Kiliae die xxvi Decem. 1776. 


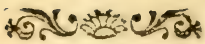 \\ Claffi $I$. \\ ELEVTERATA.}

s maxillis palpisque.

Palpi articulati, cornei faepius quatuor: anterioribus adhaerentibus maxillae dorfo, pofterioribus labio.

Interáum fex, anticis duobus brenioribus maxillae incumbentibus, cum intermediis maxillae dorfo infertis, po. fticis labio.

Clypeus horizontalis, corneus, rotundatus, os fuperne tegens.

Mandibulae duae transuerfales, corneae, mobiles latera oris fuperne includentes. Maxillac duae, liberae, transuerfales, faepius membranaceae, cumpreflae, lateraoris inferne includentes.

Labium porrectum, liberum, mox cor: neum, mox membranaceum, os inferne claudens.

Antcnuae inter oculos infertae. 
GENERA Claffis I.

I. LVCANVS.

24. NITIDVLA.

2. SCARABAEVS. 25. COCCINELLA.

3. TROX.

26. CASSIDA.

4.MELOLONTHA 27. ALVRNVS.

5. TRICH!VS.

28. CHRYSOME-

6. CETONIA.

LA.

7. HISTER.

8. APATE.

29. CRYPTOCE-

PHALVS.

9. DERMESTES.

31. CISTELA.

IO. MELYRIS.

32. CRIOCERIS.

I . BOSTRICHVS. 33. EROTYLVS,

12. BYRRHVS.

34. LAGRIA.

I3. ANTHRENVS, 35. ZYGIA.

14. ANOBIVM. 36. ZONITIS.

15. PTINVS.

16. BRVCHVS.

37. APALVS.

38. CVRCVLIO.

17. ELOPHORVS. 39. ATTELABVS.

I 8.SPHAERIDIVM. 40. CLERVS.

I9. TRITOMA.

I9. * FPS.

20. HISPA.

41. NOTOXVS.

42. SPONDYIIS.

43. PRIONVS.

2. NICROPHO-

RVS.

22. SILPHA.

23. OPATRVM.

44. CERAMBYX.

45. LAMIA. 46.STENOCORVS.

47. CALOPVS.

48. RHA- 


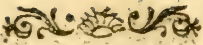

48. RHAGIVM.

49. SAPERDA.

50. CALLIDIVM.

51. DONACIA.

52. LEPTVRA.

53. LAMPYRIS.

54. PYROCHROA.

55. LYMEXYLON.

56. CVCVIVS.

57. CANTHARIS.

58. MALACHIVS,

59. NECYDALIS.

60. ELATER.

6. BVPRESTIS.

62. CICINDELA.

63. ELAPHRVS.

64. HYDROPHI-

LVS.

65. DUTISCVS.
66. GYRINVS.

67. CARABVS.

68. SCARITES.

69. SEPIDIVM.

70. PIMELIA.

7I. SCAVRVS.

72. BLAPS.

73. TENEBRIO.

74. HELOPS.

75. ERODIVS.

76. MELOE.

77. LYTTA.

78. MYLABRIS.

79. CEROCOMA.

80. MORDELLA.

81.STAPHYLINVS

82. OXYPORVS

83. PAEDERVS. 
I. LVCANVS Linné; Plarycerus Geoffroy.

Os maxillis palpisque quatuor.

$P$ alpi inaequales, filiformes articulis ob. conicis: fecundo longiore.

anteriores longiores, adhaerentes maxillae dorfo.

pofteriores fub labio abfconditi, adnati penicillis duobus, elongatis, fub filiformil u's, bafi connatis, ad bafin labii infertis.

Mastibula porrecta, cornea, arcuata, dentata.

Maxilla porrecta, membranacea, tota fetofa, medio vnidentata.

Labium porrectum, coriaceum, apice rotundatum, emarginatum, palpos pofteriores tegens.

Antennae undecim articulatae: articulo fecundo longifimo, vitimis quatuor por. recto-lamellatis.

Larwa hexapoda, annulata, nuda abdomine apice veficulofo, capite corneo.

Puppa intra glebam quiefcens abdomine 1pinis duabus caudalibus, rigidis, articulatis, mobilibus.

Victus laruae e ligno putrefcente, acidulo, imaginis e foliis arborum.

obf. Mandibulae maris in guibusdam fpeciebus longiffimas, corniformes. 


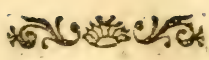

\section{SCARABAEVS Linné Geoffroy.}

\section{Copris Geoff.}

Os maxillis palpis que quatuor. $P_{a l p i}$ fubaequales, filiformes.

Anteriores vix longiores, quadriarticula. ti articulo primo bieuiffimo adhaeren. tes maxillae dorfo.

poferiores triarticulati articulis a equalibus labii apicibus prominentibus inferti. Mandibula porrecta, cornea, arcuata, inermis, apice obtufa.

Maxilia elongata, membranacea, ad infer. tionem palporum vnidentata.

Libium porrectum, corneum, fubcylindricum, apice emarginatum.

Antmnae clauatae, lamellatae, articulis vndecim: vltimis tribus lamellis porreEtis obtufis.

Larua hexapoda, annulata, pilofa, abdomine fubtus veficulofo, capite corneo. Puppa intra glebam quiefcens, abdomine veficulofo, ecaudato.

Victus laruae $2 x$ imaginis e 1 tercore animalium.

Obf. I. Mandibula Scarab. Thyphoei apice tridentata.

2. Maxilla in quibusdam fpeciebus apice dilatata, rotundata.

3. Copris Geoffroy tantum defectu fcutelli differt.

4. Antennae rarius lamellis feptem. 
6 เลำ

3. TROX. Scarabaeus Linné.

Os maxillis palpisque quatuor.

Palpi inaequales, capitati.

anteriores longiores, quadriarticulati, articulis aequalibus; vltimo oblongo craffiori adhaerentes maxillae.

pofteriores triarticulati: articulo vltimo oblongo, craffiori labii apici inferti.

Mandibula breuis, cornea, craffa, obtufa; edentula.

Maxilla cylindrica, membranacea, recta; bifida : laciniis fubaequalibus acutiufculis, fetofis.

Labium porrectum, coineum, rotundatum, apice membranaceum, emarginatum.

Antennas breues: articulo primo craffioris pilofo, vltimis tribus ouatis, lamellatis. Metamorphogis \& Victus ignotae. 


\section{ตำ}

\section{MELOLONTHA. Scarabacus Linné. Geoffr.}

Os maxillis palpisque quatuor.

Palpi inaequales, filiformes.

anteriores longiores, quadriarticulati : articulo primo tertioque breuifimis, quarto longiori oblongo adhaerentes maxillae dorfo.

poferiores breues, triarticulati: articulis fubaequalibus labii parieti interiori inferti.

Mandibula breuis, cornea, arcuata, apice compreffa, acutiufcula, vix dentata.

Maxilla cornea, breuis, rigida, fubarcuata, apice obtuí, multidentata.

Labium porrectum, corneum, cordatum, apice fubemarginatum.

Antennae breues clanato lamellatae: articulo primo globofo, craffiori.

Larua hexapoda, annulata, fubpilofa, ab. domine apice veficulofo, capite corneo. Puppa quiefcens intra glebam, abdomine apice acuminato.

Victus laruae e plantarum radicibus, imaginis ex arborum foliis.

06f. I. Melolonthae folftitialis labium magis emarginatum quam in reliquis.

2. Antennarum claua in quibusdam lamellas feptem gerit. 
5. TRICHIVS. Scarabaeus Linné.

Os maxillis palpisque quatuor.

Palpi aequales, filiformes: articulo vltimo longiori.

anteriores quadriarticulati, adhaerentes maxillae dorfo.

pofteriores triarticulati medio laterali la. bii inferti.

Mandibula cornea, craffa, obtufa, edentula.

Maxilla cylindrica vsque adbafin bifida: laciniis aequalibus, obtufufculis apice fetofis.

Labium cylindricum, elongatum, corne$\therefore$ um, emarginatum: apicibus aequalibus, acutiufculis.

Antennae clauato-lamellatae: lamellis tribus ouatis, articulo primo craffiori pilofo.

Metamorpbofis ignota.

Imago delectatur floribus inprimis vm. bellatarum.

Obf. Trichius fafciatus labio gaudet hirfuto. 


\section{*⿻ำ 눈}

6. CETONIA. Scarabaeus Lintr.

Geoff.

Os maxillis palpisque quatuor.

$P$ alpi fubaequales.

anteriores filiformes, triarticulati: ar: ticulo vltimo cylindrico adhaerentes maxillae.

poffcriores triarticulati: articulo vltimo longiori, fubcraffiori bafi internae labii inferti.

Mandibula porrecta, cornea, recta, acu. ta, inermis.

Maxilla ad infertionem palporum dilatata, vnidentata, fetofa.

Lsbium elongatum, coriaceum, cylindricum, apice fiffum, aut emarginatum palpos pofteriores fere totos tegens.

Antennae breues, clauato lamellatae; lamellis tribus ouatis, articulo primo porrecto, craffiori.

Larua hexapoda, annulata, pilofa, abdomine apice veficulofo, capite paruo corneo.

Puppa quiefcens intra glebam, abdomine apice obtufo.

Victus laruae e ligno putrefcente, fubacidulo, imaginis ex arborum foliis \& floribus.
A 5
7. HI- 


\section{HISTER. Linn. Attelabus}

\section{Geoff.}

Os maxillis palpis que quatuor.

Palpi aequales, fubfiliformes: articulis oblongis, aequalibus.

antcriores quadri articulati: articulo vltimo obtufo, truncato fub apice maxillae inferti.

pofteviores triarticulati apici labii adhaerentes.

Mandibula porrecta, cornea, arcuata, acuta, vnidentata.

Maxilla membranacea, fubcylindrica, vnidentata, apice obtufa.

Labium corneum, porreftum, cylindricum, apice membranaceum, rotunda. tum, integerrimum.

Antennac breues, vndecim articulati: articulo primo longiori, reliquis fubglobofis vltimo clauato, ouato.

Metamorpbojis \& victus latent. 


\section{*त्रण सै}

\section{A PA TE. Dermeftes Linn. Ligni- perda Pall.}

Os maxillis palpisque.

Palpi quatuor aequales, filiformes.

anteriores quadriarticulati: articulo vltimo breuiori, cylindrico, obtufo adhaerentes maxillae dorfo.

Poferiores triarticulati: articulo vltimo breuiori, craffiori, obtufiffimo labii medio inferti.

Mandibula cornea, recta, acuta, bafi dentata.

Maxilla membranacea, ad infertionem palpi vnidentata, apice rotundata.

L̇abium breue, cylindricum, membrana. ceum, depreffum, obtufiffimum \& fere truncatum, ciliatum, integerrimum.

Antennac clauatae: claua articulis tribus diftantibus, perfoliatis.

Metamorphofis ignota.

Victus imaginis e ligno putrefcente. 
8. DERMESTES. Linn Geaf: Clerus. Geoff.

Os maxillis palpisque.

Palpi quatuor inaequales, filiformes. anteriores longiores, quadriarticulati : articulis aequalibus adhaerentes maxillae dorfo.

pofteriores triarticulati articulo vltino maiori fub labii apice interiori inferti.

Mandibula cornea, arcuata, acuta, inermis.

Maxilla cylindrica apice rotundata, obtufiffima, bifida longinudine palporum anteriorum: laciniis fubacqualibus; $\mathrm{ex}$ teriore paullo maiore.

Labium fubcylindricum, corneum, clongatum, obtufum, integerrimum palpos inferiores fere tegens.

Antenna articulo primo maiori, craffiori, vitimis tribus clauato perfoliatis.

Larua hexapoda, fafciculato pilofa cauda faepius fetofa.

Puppa quiefcens, immobilis, hifpida cauda obtufa.

Vidtus laruae \& imaginis e pinguedine, pellibus \&rc. 


\section{* \\ IO. MELYRIS.}

Os maxillis palpisque.

Palpi quatuor aequales, filiformes.

anteriores quadriarticulati : articulis fubaequalibus; vltimo ouato adhaeren. tes maxillae dorfo.

poferiores triarticulati: articulo vltimo ouato labii medio laterali inferti.

Mandibule breuis, cornea, incurua, edentula.

Maxilla breuis, fubcornea, inmedio vni dentata, apice acutiffima.

Labium porrectum, cylindricum, corne. um, annulatum, apice membranaceum, clauatum, emarginatum.

Anienrae fecundum totam longitudinem perfoliatae: articulis breuibus, vtrinque pilofis, vltimo ouato, obtufo.

Metamorpholis \& viçus latent. 
Ir. BOSTRICHVS. Geoff. Dermeftes. Linn. Scolytus Geoff.

Os maxillis palpisque.

Palpi quatuor aequales.

anteriores in medio craffiores, triarticu. lati: articulis fubaequalibus adhaeren. tes maxillae.

pofferiores rriarticulati: articulis aequalibus labii apici inferti.

Mandibula breuis, cornea, craffa, forni. cata, acuta, inermis.

Maxilla cornea, breuis, craffa, recta, rigida, cylindrica, acutiulcula, inregra.

Labium porrectum, membranaceum, tenue, cylindricum, integrum.

Antennae vndecim articulatae: articulo primo paullo longiori, fequentibus breniffimis, rotundatis, vltimis tribus elongatis incraffatis, ouatis vltimo acuto.

Metamorphofis mihi haud rite nota.

Victus \& laruae \& imaginis e ligno ramorum emortuorum.

Obf. B. Scolytus antennis apice obtufis differt.

12. BYRR- 
12. BYRRHVS Linn.

Os maxillis palpisque.

Palpi quatuor aequales, fubclauati: articulis aequalibus; vltimo fubrotundo, cratfiori,

anteriores quadriarticulati maxillae dor. fo adhaerentes.

pofterioves triarticulati labii medio adhaerentes.

Mandibula breuis, craffa, cornea, recta, dentata, apice filia.

Maxilla membranacea, bifida: lacinia exteriore maiore rotundata.

Labium membranaceum, porrectum, rotundatum apice bifidum: laciniis aequalibus, conniuentibus.

Antenuac primo articulo longiori, reliquis breuifimis perfoliatis fenfim craffioribus.

Metamorphofis \& Victus adhuc omnino latent. 
I3. ANTHRENUS. Geoff. Byrrhus Linn.

Os maxillis palpisque.

Palpi quatuor inaequales, filiformes.

anteriores longiores, quadriarticulati: articulis aequalibus, cylindricis adhaerentes maxillae dorfo.

pofteriores cylindrici, breuiffimi, obtufi, triarticulati: articulis aequalibus fub labii apice interiori inferti.

Mandibula cornea, arcuata, acuta, inermis.

Maxilla membranacea, linearis, obtufa, bifida: lacinia exteriori fub longiori.

Labium breue, corneum, rotundatum, integrum palporum pofteriorum bafin tegens.

Antennae cylindrico clauatae: articulis bre. uiffimis, vltimis tribus incraffatis, connatis clauam ouatam, folidam conftituentibus.

Larua hexapoda, villofa.

Puppa immobilis cauda obtura

Vič̉us laruae e plantis emortuis, imaginis e floribus. 


\section{भล⿵人一}

\section{7}

14. ANOBIV M. Ptinus Linn. Dermertes Linn. Byrrhus Geoff.

Os maxillis palpisque.

Palpi quâtuor, fubacquales, clauatì.

smieriores parum longiores, quadriartis culati: articulo fecundo longiori adhaerentes maxillae dorfo.

pofteriores triarticulati: articulis fubaequa. libus labii apici inferti.

Mandibuta breuis, cornea arcuata, acu. ta, inermis.

Maxilla breuis, cylindrica, crafta, cor: nea, recta, integra, apice obtufas dentata.

Labium breue, cylindricum, membranaceum apice truncatum, integerrimum:

Antennaic filiformes: articulis anterioribus orbiculatis, vltimis tribus ouatis bafi attenuatis:

Larua hexapoda, nuda, mollis capite callofo.

Puppa immobilis.

Victus lariae e ligno duriori; incorrupto. 


\section{*ते}

15. PTINVS Linn. Bruchus Geoff. Bupreftis Scop.

Os maxillis palpisque.

Palpi quatuor, aequales, filiformes.

anteriores quadriarticulati: articulis aequalibus vitimo fetaceo adhaerentes maxillae dorfo.

poferiores triarticulati: articulis aequalibus adnati labii apici laterali.

Mandibula cornea, arcuata, comprefra.

Maxilla porrecta, membranacea, cylindrica, obtufa, bifida: laciniis cylindricis obtufis, aequalibus.

Iabium porrectum, membranaceum, cylindricum, ad bafin bifium: laciniis linearibus, cuius apicibus fummis palpi pofteriores infident.

Antenna longiores, filiformes: articulis obconicis; lecundo globolo.

Iarua hexapoda, mollis, glabra.

Pupp folliculata, immobilis.

Vifus \& laruae \& imaginis e plantis \& animalibus exficcatis at haut putre. fcentibus. 


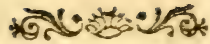

16. BRVCHVS Linn. Mylabris Geoff.

Os maxillis palpisque.

Palpi quatuor, fubaequales, filiformes.

anteriores vix longiores, guinque articulati : articulis a equalibus; vltimo cy. lindrico adhaerentes maxillae dorfo.

poferiores quadriarticulati: articulis aequalibus; vltimo globofo labii me. dio laterali inferti.

Mandivula vix arcuata, cornea, inermis.

Maxilla porrecta longitudine palporum, membranacea, cylindrica, bifida: la. ciniis conniuentibus, aequalibus.

Labium membranaceum, breue, inter pal. pos acuminatum, integerrimum.

Antennae elongatae, fubfiliformes: articulis introrfum apice prominulis.

Metamorphofis omnino adhuc latet.

Victus imaginis e feminibus plantarum. 
17. ELOPHORVS. Silpha Linn. Dermeftes. Geofi:

Os maxillis palpisque.

Palpi quatuor, rubaequales.

anteriores quadriarticulati: articulo fecundo longifirno, vltimo fubcrafliori adhaerentes maxillae.

pofferiores triarticulati articulo vltimo fubcraffiori labii apici inferti.

Mandibula cornea, arcuata, acuta, edentula. Maxilla cornea, cylindrica, adinfertionem palporum vnidentata, apice nembranacea, fetofa aur potius fiffa.

Labium porrectum, corneum, çuadratum, truncatum, integrum.

Antenna brenes, clauatac: claua folida articulis tribus crafioribus.

Metamorphofis ignota.

Vicius imaginis e foliis plantarum aquaticarum. 


\section{I8. SPHAERIDIVM.}

Dermeftes Linn. Geoff. Silpha Linn. Anthribus Geoff.

Os maxillis palpisque.

Palpi quatuor inaequales, filiformes.

anteriores longíores, quadriarticulati: ar. ticulo fecundo maiori adhaerentes maxillae.

pofieriores breuiffimi, triarticulati fub labii apice inferti.

Mandibula cornea, arcuata, acutiffima, inermis.

Maxilla porrecta, fubarcuata, apice membranacea, rotundata, bifida: laciniis fubaequalibus, obtufis.

Labium elongatum, corneum, quadratum, apice emarginatum, ciliatum.

Antennac clauatae : claua perfoliata e articulis tribus incraffatis conftans.

Metamorphofis ignota.

Victus imaginis e ftercore animalium. 


\section{TRITOMA. Geoff.}

Os maxillis palpisque.

Palpi quatuor, inaequales.

anteriores multo longiores, fecuriformes, triarticulati: articulo fecundo breuiffimo, vltimo claua dilatata, acuminata adhaerentes maxillae dorfo.

pofteriorcs breues, incurui, biarticulati: articulo vltimo fubcraffiori, obtufo fub labii apice inferti.

Mandibula cornea, arcuata, inermis, apice fiffa.

Maxill breuis, membranacea, cylindrica, bifida: laciniis aequalibus, filiformibus.

Labium porrectum, cylindricum, bafi corneum, apice membranaceum, fube. marginatum.

Antennae clauatae: claua perfoliata articu.

lis tribus craffioribus conftans.

Metamorphofis ignota.

Victus imaginis e fungis inprimis Boletis arborum. 


\section{*ख)}

19. * IPS. Silpha Linn. Dermeftes Geoff.

Os maxillis palpisque.

Palpi quatuor, breuifimi, aequales, fili. formes.

anteriores triarticulati vix maxillae longiores: articulo vltimo obtufo, truncato adhaerentes maxillae.

pofterieres triarticulati, breues: articulo vltimo obtufo, truncato labii medio laterali inferti.

Mundibula breuis, cornea, vix arcuata, inermis.

Maxilla breuis, membranacea ,bifida: laciniis fubaequalibus, linearibus; interiore paullo breuiore.

Labium breuiffimum, corneum, truncatum, emarginatum.

Antcnrac porrectae, clauatae: articulis rotundatis, aequalibus; vltimis tribus craffioribus, perfoliatis.

Metamorphofis ignota.

Victus imaginis e cadaueribus animalium.

obf. Genus affine Sphaeridio. 
20. HISPA. Linn. Dermeftes Iinn, Ptinus Linn.

Os maxillis palpisque.

Palpi quatuor, breues, aequales, fubfili: formes.

mteriores quadriarticulati articulis rub. aequalibus adhaerentes maxillae.

poferiores triarticulati: articulis aequali. bus labii baị laterali inferti.

Mandibula cornea, arcuata, acuta, inermis. Maxilla breuis, cylindrica, membranacea, bifida: laciniis aequalibus, filifor: mibus.

Labium porrectum, membranaceum, cylindricum, apice truncatum, incegrum,

Antennac cylindricae: articulis breuibus bafi apiceque planis.

Larua hexapoda, nuda.

Puppa immobilis, obtura.

Vichus Laruae e lignoarborum putreficente.

Qbf. T. Antennae cylindricae, plerisque fpeciebus pectinatae, in quibusdam fimplices, interdum extrorfum craftiores. Qb. II. Genus Anobio affine. 


\section{NICROPHORVS.}

Silpha Linn. Dermeftes Geoff.

Os maxillis palpisque.

Palpi quatuor, aequales, filiformes.

anteriores quadriarticulati: articulis aequalibus; vltimo cylindrico adhae. rentes maxillae dorfo.

pofteriores quadriarticulati: articulo primo longiffimo, vltimo globofo la. bii bafi exteriori inferti.

Mandibula cornea, arcuata, acuta, inermis. Maxilla recta, bafi vnidentata, apice onata, rotundata, integra longitudine palporum.

Labium fub palpis elongatum, membranaceum, cordatum, apice emarginatum, crenatum,

Antemac clauatae: articulo primo longiflmo, fequentibus breuibus, penultimis tribus craffioribus, perfoliatis, transverfis, vltimo ouato, acuminato.

Larma hexapoda, nuda, capite calloro.

Puppa fubpilofa, quiefcens, ano ftylis duobus, incuruis.

Vicut e cadaueribus animalium.

$$
\text { B } 5 \text { 22. SIL }
$$


22. SILPHA Linn. Peltis Geoff.

Os maxillis palpisque.

Palpi quaruor, inaequales, filiformes.

anteriores quadriarticulati: articulo fecundo obconico, vitimo cylinćrico ad. haerentes maxillae dorfo.

pofteriores triarticulati: articulo vltimo obo. vato labii bafi exteriori inferti.

Mandibula breuis, cornea, compreffa, incurua, obtufa, inermis.

Maxilla ad infertionem palporum dente porrecto, corneo, valido, arcuato, acuto armata, apice membranacea, rotundata, ciliata.

Labium elongatum, membranaceum, obtufum, fiffum.

Antennac clauatae; articulo primo paulo longiori, fequentibus breuibus apicibus prominulis, guatuor penultimis craflioribus, perfoliatis, vltimo onato.

Larua hexapoda, nuda, depreffa, duriufcula marginibus fegmentorum vtrinque prominulis fubfpinofis.

Puppa ouata, nuda, quiefcens.

Victus \& Laruae \& Imaginis e animalium cadaueribus. 


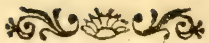

\section{OPA TRUM. Silpha. Linn. Tenebrio Geoff.}

Os maxillis palpisque.

$P_{a l p i}$ quatuor, inaequales, clauati.

Antcriores longiores, quadriarticulati : articulis aequalibus; vltimo craffiori, obtufo, truncato adhaerentes maxillae dorfo.

poferiores triarticulati, extrorfum craffio. res labii lateribus inferti.

Mandibula cornea, arcuata, acuta, inermis.

Maxilla breuis, cylindrica, membranacea, bifida: laciniis inaequalibus, acutis; interiore breuiore.

Labium corneum, orbiculatum, porrectum, apice membranaceum, fubemarginatum.

Antennac moniliformes, extrorfum craffio. res: articulis fubaequalibus.

Mctamorphofis \& victus \& laruae \& imaginis ignota. 
28

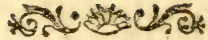

24. NITIDULA. Silpha Linn. Dermeftes Geoff.

Os maxillis palpisque.

Palpi quatuor, aeguales, filiformes. anteriores quadriarticuiati:articulis aequalibus adhaerentes maxillae dorfo.

poferiores triarticulati: articulis aequalibus labii medio laterali inferti.

Mandibula cornea, arcuata, acura, inermis.

Maxilla longirudine palporum cylindrica, apice acita, integra.

Labium elongarum, membranaceum, cylindricum, apice rotundatum, integerrimum.

Antennae clamatae: articulis breuibus, fubaequalibus; vltimo onato, craffiori.

Metamorphofis \& viceus ignota. 


\section{COCCINELLA. Linn. \\ Geoff.}

Os maxillis palpisque,

Palpi quaruor, inaequales.

antcriores paullo longiores, fecuriformes, triarticulati : claua dilatata acuta adhacrentes maxillae dorfo.

pofferiores filiformes, biarticulati : articulis aequalibus labii mectio inferti.

Mandibula cornea, arcuata, acuta, eden. rula.

Maxilla cylindrica, obtufa, recta, bifida: laciniis fubacqualibus, membranaceis.

Labium rectum, porrectum, cylindricum, ad infertionem palporum coarctatum, apice roturdatun, integrum.

Antenne clauatae: clanafolida, articulo primo longion, vlimis quatuor incraffatis vltimo fubacuminato.

Larua hexapoda, fubuerrucofa, poftice attenuata.

Puppa gibbofa, fubroturida, immobilis. Victus \& laruae \& imaginis e rapina A. phidum. 
26. CASSIDA. Linn. Geoff.

Os maxillis palpisque.

Palpi quatuor, inaequales.

anteriores longiores, quadriarticulati: articulo fecundo longiori, vltimo cla. uato maxillae dorfo adnati.

pofteriores breuifimi, filiformes: articulis rotundatis, aequalibus labii bafi inferti.

Mandibula cornea, arcuata, acuta, edentula.

Maxilla membranacea, cylindrica, obtufa, integerrima.

Labium elongatum longitudine palporum pofteriorum, cylindricum apice incralfatum, obtufum, integerrinum.

Antennae moniliformes, extrorfum craffiores: articulis fubaequalibus, vltimo obouato.

Larua hexapoda, depreffa, fubrotunda, bicaudata, lateribus fpinofo ciliatis.

Puppa breuis, ouata, depreffa lateribus fpinofo ciliatis, quiefcens.

Victus \& laruae \& imaginis e foliis plan. tarum in primis Carduorum. 


\section{+बत \\ 27. ALURNUS.}

Os maxillis palpisque.

Palpi fex, inaequales.

anteriores breuifimi, maxillae multo

breuiores, biarticulati: articulo fecundo rotundato, ciliato adhaerentes maxillae dorfo.

intermedii longiores, filiformes, quadriarticulari: articulis fubaequalibus ad bafin anteriorum adnati.

pofferiores fubfiliformes, triarticulati: articulo primo breuifimo, fecundo obconico, tertio rotundato parum craffiori fub labii inferioris apice in. ferti.

Mandibula craffa, cornea, arcuata, acuta, in medio vnidentata.

Maxilla porrecta palpis anticis mul to longior, cornea, fornicata, apice rotundata, ciliaca.

Labium corneum, rotundatum, apice acuminatum, integerrimum.

Antenrae filiformes: articulis cylindricis; fecundo longiori, reliquis aequalibus, vltimo acuminato.

Mctamorphofis \& victus ignota.

28. CHRY- 
28. CHRYSOMELA. Linn. GCOff. Altica Syft. Ent. Geoff.

Os maxillis palpisque:

Palpi fex, inaequales.

anteriores longitudine maxillae filifor: mes, biarticulati : articulis aegualibus adhaerentes maxillae dorfo.

intermedii longiores : articulo primo breuiffimo, fecundo longiori, vlrimo craffiori, truncato ad bafin ante: riorum adnati.

poferioves triarticulati : articulo fecun-

do longiori, vltimo crafliori, trun. cato labii medio inferti.

Mandibula porrecta ${ }_{2}$ cornea, arcuata, acuta, edentula.

Maxilla breuis, recta, membranacea, co. nica, acuta, integra.

Labium breue, coineum, rotundatum, fubcompreftum, integerrimum.

Antman moniliformes: articulis vndecirn fubrequalibus; vltimo ounto.

Larua hexapoda, mollis, nuda capite callofo.

Puppa brevis, ouata, guiefecns.

Vickus \& laruae \& imaginis e foliis plan. tarum.

Obf. Altica f. Chryfomelae faltatoriae Linn. gerte huius generis. 
29. CRYPTOCEPHALVS. Geoff.

Chryfomela Linn. Melolontha Geoff.

Bupreftis Scop.

Os maxillis palpisque.

Palpi quatuor filiformes, aequales.

anteriores quadriarticulatiarticulis aequa-

libus adhaerentes maxillae, incumbentes.

poperiores triarticulati: articulis aequalibus apici labii inferti.

Mandibula breuis, craffa, cornea, difformis, dentata.

Maxilla longitudine palporum, membranacea, filiformis, bafi dente elongato, rigido longitudine dimidiae maxillae armata.

Labium elongatum, corneum, cylindricum, apice rotundatum, integerrimum.

Antennac filiformes articulis vndecim, primo breuiori globofo, reliquis obconicis interne ferratis.

Larua hexapoda, mollis, nuda.

Puppa ouata, immobilis.

Victus \& laruae \& imaginis e foliis plan. tarum.

obf. Melolontha Geoff. antennis tantum ferratis differt.

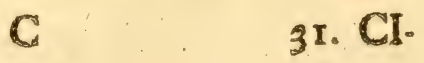


3r. CISTELA. Geoff. Chryfomela Linn. Mordella Geoff.

Os maxillis palpisque.

Palpi quatuor, inaequales, filiformes.

anteriores longiores, quadriarticulati: articulo primo breuiffimo, fecundo longiori adhaerentes maxillae dorfo.

pofferiores quadriarticulati: articulis breuiffimis fubaequalibus labii medio laterali inferti.

Mandibula cornea, arcuata, fubintegra.

Maxilla porrecta, membranacea, cylindrica ad infertionem palporum vnidentata, apice obtufa.

Labium porrectum, membranaceum, cylindricum adinfertionem palporum coarctatum, apice bifidum: laciniis aequalibus, linearibus, diftantibus.

Antennue filiformes: articulis vndecim, obconicis apice fubprominulis.

Metamorpbofis ignota.

Victus imaginis e floribus \& foliis plantarum. 
32. CRIOCERIS. Geoff. Chryfomela Linn. Galeruca Geoff.

Os maxillis palpisque.

Palpi quatuor, breues, aequales, fubfiliformes.

anteriores quadriarticulati: articulo vltimo parum maiori, craffiori apice acuminato adhaerentes maxillae bafi.

pofferiores triarticulati, breues: articulis fubaequalibus, vltimo acuminato labii medio inferti.

Mandilula cornea, arcuata, acuta, inermis.

Maxilla porrecta, recta, obtufa, bifida: laciniis aequalibus longitudine palporum anceriorum.

Labium breuifimum, membranaceum, gibbum, apice rotundatum, integrum. Antennac filiformes, articulis vndecim, obconicis, apice aequalibus; fecundo maiori.

Lurua hexapoda, nuda, mollis, punctis eleuatis fcabra.

Puppa quiefcens, ouata, gibba, fcabra. Victus \& laruae \& imaginis e foliis plantarum.

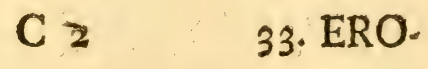


33. EROTYLVS. Chryfomela Linn.

Os maxillis palpisque.

Palpi quatuor, inaequales.

anteriores longiores, fecuriformes, quadriarticulati: articulo tertio breviffimo, vltimo craffiori, oblique truncato adhaerentes maxillae dorfo.

pofteriores breuiffimi, fubclauati, triarti. culati: articulo fecundo breuiftimo, vltimo parum craffiori, truncato in. ferti labii medio laterali.

Mandibula cornea, concaua, acuta, edentula.

Maxilla cornea, bifida: lacinia cxteriore maiore, clauata, concaua, rotundata.

Labium breue, corneum, bafi cylindricum, apice dilatatum, truncatum, fube. marginatum.

Antennac filiformes: articulis vndecim, fubaequalibus.

Metamorphofis \& Victus \& laruae \& ima. ginis incognita. 


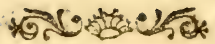

34. LAGR IA. Chryfomela Linn. Meloe Linn.

Os maxillis palpisque.

Palpi quatuor, inaequales.

anteriores multo longiores, fecuriformes, quadriarticulati : articulis aequalibus, vltimo dilatato, acuminato adhaerentes maxillae dorfo.

poiteriares breuilimi, extrorfum crafico. res, triarticulati: articulo vlcimo craífiori, truncato labii medio inferti.

Mandibula cornea, breuis, arcuata, vnidentata.

Maxilla cylindrica, membranacea, bifida: laciniis inaequalibus; exteriore maiore rotundata.

Labium breue, rotundatum, membranaceum, integrum.

Antennae filiformes: articulis vndecim obconicis; primo clauato, longiori, fecundo breuifimo, globofo, vltimo ouato, obrufo.

Metamerphofis ignota.

Vičtus laruae ignotus, imaginis e foliis arborum.

$$
\mathrm{C}_{3} \quad 35 \mathrm{ZY}-
$$




\section{*ล⿻上丨冖 \\ 35. ZYGIA.}

Os maxillis palpisque.

Palpi quatuor, inaequales, filiformes.

anteriores longiores, quadriarticulati: articulo vltimo longiori, fetaceo ad. haerentes maxillae dorfo.

poffcriores breuiores, triarticulati : articulo primo breuiffimo, reliquis cy. lindricis labii medio anteriori inferti.

Mandibula cornea, arcuata, acuta, integra.

Maxilla reeta, membranacea, compreffa: bafi dente ollato, breui apice outa rotundata.

Labium valde elongatum, membranaceum, cylindricum, ad infertionem palporum parum coarctatum, apice truncatum, vix emarginarum.

Anternac moniliformes, extrorfum craffiores: articulis fubaequalibus primo paullo craffiori, reliquorumque apicibus prominulis.

Metamorphofis \& Victus \& laruae \& ima. ginis omnino ignota. 


\section{*مै। \\ 36. ZONITIS.}

Os maxillis palpisq̧ue.

Palpi quatuor, inaequales, filiformes.

anteriores longiores, quadriarticulati: articulo fecundo longiffimo, vltimo obtufo adhaerentes maxillae dorfo.

pofteriores breuiores, triarticulati: articu. lo fecundo longiflimo labii medio anteriori inferti.

Mandibula cornea, arcuata, comprefla, acuta, inermis.

Maxilla porrecta longitudine dupla palporum anteriorum, fetacea, fubmembranacea, ciliata, integra.

Labium porrectum, membranaceum, cylindricum, apice villofum, emargi. natum.

Antennae fetaceae, elongatae: articulis cylindricis, fubaequalibus.

Mctamorplofis \& Vietus \& laruae \& imaginis latent. 


\section{APALVS. Meloe Linn.}

Os maxillis palpisque.

Palpi quaruor, aequales, filiformes.

snteriores quadriarticulati : articulis fubaequalibus adhaerentes maxillae dorfo.

pofteriores triarticulati: articulis longioribus, obconicis labii medio inferti.

Mandibula cornea, arcuata, acuta, iner. mis.

Maxilla cornea, recta, fubcylindrica ad infertionem palporum vnidentata, apice obtufa, rotundata.

Iabium porrectum, fubmembranaceum, cylindricum, apice truncatum, obtufum, integrum.

Antenuac filiformes: articulis vndecim, obconicis, fubaequalibus; vltimo ouato, obtufo.

Metamorplofis \& Victus \& laruac \& ima. ginis adhuc latent. 
42 38. CVRCVLIO. Linn. Geoff.

Os roftro elongato, corneo maxillis palpisque.

Palpi quatuor, breuilimi, aequales, fili-, formes.

antcriores quadriarticulati: articulis aequalibus, breuiffimis ; vltimo acuto adhaerentes maxillae dorfo incum. bentes.

pofferiores triarticulati labii apice infidentes.

Mandibula breuis, cornea, arcuata, acuta, inermis.

Maxilla breuis, cylindrica, bafi vnidentata, apice acuta longitudine palporum.

Labium porrectum, cylindricum, apice rotundatum, membranaceum, inte-

gerrimum.

Antcnise infidentes roftro corneo plerum. que clauatae.

Laru hexapoda, mollis, annulata, albida capite pedibusque callofis.

Puppa immobilis.

Victus laruae e feminibus, difco \& foliis plantarum, imaginis e foliis.

Obf. Antennae differunt \& forfitan meliores darent fpecierum iubdiufiones, quas vero nondum affequi valui.

a. Antentac clatatae: articulis aequalibus.

b. Antennae clauntae: articulo prino longifimo.

c. Antennae moniliformes.

$$
\text { C } 5 \text { 39. AT. }
$$


39. A T TELABVS. Linn. Rhinomacer Geoff.

Os roftro elongato corneo maxillas palpos. que continente.

Palpi quatuor, inaequales, filiformes.

antcriores longiores, quadriarticulati: articulis aequalibus, rotundatis adhae. rentes maxillae dorfo.

pofteriores breuiores, triarticulati : articu. lis rotundatis, aequalibus labii parieti interiori inferti.

Mandibula cornea, arcuata, acuta, fubinermis.

Maxilla porrecta, cylindrica, membrana. cea, bifida: laciniis aequalibus, acutis.

Labium breue, latum, corneum, truncatum, crenatum palpos inferiores totos tegens.

antennae moniliformes: articulo primo longiori, clauato, reliquis aequalibus breuiffimis, vltimo clauato, obtufo, ouato.

Larua hexapoda, mollis, annulata. Puppa immobilis, ouata, laeuis. Victus \& laruae \& imaginis e foliis plantarum. 


\section{CLER V S. Geoff. Attelabus Linn.}

Os maxillis palpisque.

Pulpi quatuor, aequales.

anteriores breuiores, filiformes, quadriarticulati: articulo viltimo obconico adhaerentes maxillae dorfo.

pofteriores longiores, clauati: clạua dilatata, fecuriformi, articulis reliquis aequalibus labii medio inferti.

Mandibula cornea, arcuata, acuta, edentula. Maxilla cornea, recta ad infertionem pal. porum vnidentata apice rotundata.

Labium elongatum, membranaceum ad infertionem palporum coarctatum, apice dilatatum, emarginatum.

anternae moniliformes: articulo primo crafícri, longiori, reliquis moniliformibus, vltimis tribus annulatis, craffioribus.

Larua hexapoda, mollis, annulata. Puppa folliculata, quiefcens.

Victus laruae e rapina laruarum, imaginis e foribus praefertim vmbellatis. 
41. NOTOXVS. Geof. Attelabus I.inn. Meloe Linn. Clerus Geoff.

Os maxillis palpisque.

Palpi quatuor, inaequales, clauati: claua fecuriformi.

anteriores longiores, quadriarticulati : articulo vltimo fecuriformi, reliquis aequalibus adhaerentes maxillae.

pofteriores triarticulati: articulo vltimo, fecuriformi labiibafi exteriori inferti.

Mandibula cornea, arcuata, acuta, edentula. Maxilla recta, cylindrica, in medio vnidentata, apice rotundata, integra.

Labium elongatum, cylindricum, mem. branaceum, rectum, apice bifidum: laciniis aequalibus, conniuentibus, obtufis.

Antenna filiformes: articulis fubcylindricis, aequalibus; exterioribus tribus breuioribus fubrotundis, vltimo ouato.

Metamorphofis ignota.

Victus larua ignotus, imaginis e floribus plantarum vmbellatarum. 
42. SPONDYLIS. Attelabus

Linn.

Os maxillis palpisque.

Palpi quatuor, aequales, fubfiliformes.

anteriores filiformes, quinque articulati: articulis aequalibus adhaerentes maxillae dorfo.

pofteriores fubclauati, triarticulati : articulis aequalibus, vltimo fubcraffiori labii bafi exteriori inferti.

Mandibula porrecta, exferta, forcipata, cornea, acuta denticulata.

Maxilla breuis, craffa, conica, acuta, recta, pilofa, integra.

Labium porrecum, corneum, bifidum: laciniis aequalibus, diftantibus, obtufis cum mucrone, vel potius cordato emarginatum cum mucrone.

Antennae moniliformes: articulis fúbaequalibus apice obtufiffimis \& fere truncatis.

Mctamorphofis ignota.

Viftus laruae ignotus, imaginis e fungis arborum. 


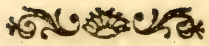

\section{PRIONUS. Geoff. Cerambyx Linn.}

Os maxillis palpisque.

Palpi quatuor, fubaequales, filiformes.

anteriores paullo longiores, quadriarticulati : articulo fecundo longyfimo, vltimo truncate adhaerentes maxillae dorfo.

poftcriores triarticulati: articulo fecundo longiffimo, vltimo truncato fublabii apice inferti.

Mandibula porrecta, cornea, exferta, ar. cuata, acuta, apice dentata.

Maxilla breuis, cornea, cylindrica, fub. incraffata, obtufa, integerrima, extrorfum ciliata.

Labium breuiffimum, membranaceum, rotundatum, integrum.

Antennae elongatae, fetaceae: articulo fecundo breuiffimo, reliquis fubaequalibus, obconicis apice prominulis.

Larua hexapoda, mollis, annulata.

Puppa immobilis.

Victus \& laruae \& imaginis e ligno putrefcente. 


\section{*बत cas}

44. CERAMBYX. Linn. Geoff.

Os maxillis palpisque.

Palpi quatuor, aequales, filiformes.

anteriores quadriarticulati : articulis breuibus, vltimo longiori, fetaceo adhaerentes maxillae dorfo.

poferiores triarticulati: articulis inaequalibus labii medio exteriori inferti.

Mandibula exferta, cornea, arcuata, acuta, edentula.

Maxilla porrecta, membranacea, fubarcuata, bafi vnidentata, apice rocunda. ta, obtufa, integra.

Labium porrectum, membranaceum, vsque ad infertionem palporumbifidum: laciniis aequalibus, rotundatis.

Antennac elongatae, fetaceae : articulis vndecim cylindricis.

Larua hexapoda, mollis, annulata capire pedibusque callofis.

Puppa intra globum quiefcens, pone acuminata.

Victus \& laruae \& imaginis e ligno pu. trefcente, acidulo. 
45. LAMIA. Cerambyx Linn.

Cerambyx Geoff.

Os maxillis palpisque.

Palpi quatuor, fubaequales, filiformes.

anteriores paullo longiores, quadriarticulati, articulo vltimo fetaceo adhaerentes maxillae.

poftcriores triarticulati, articulis aequali. bus, vltimo fetaceo labii bafi interiori inferti.

Mandibula cornea, fornicata, acuta, edentula.

Maxilla breuis, cornea, recta, bifida: laciniis inaequalibus; exteriorelongiore, tenuiore, acuta, interiori craffiori, obtufa.

Antenne clongatae, fetaceae: articulis cylindricis; primo parum crafliori, vl. timo fubacuto.

Metamorpholis ignota.

Victus \& laruae \& imaginis e ligno arbo. rum putrefcente, acidulo. 
46. STENOCORUS. Geoff.

Cerambyx Linn.

os maxillis palpisque quaruor.

Palpi quatuor, inaequales.

anteriores longiores, fubfiliformes, qua. driarticulati: arriculo fecundo quartoque longioribus maxillae adhae. rentes,

poferiores clauati, triarticulati: articulo vltimo claua obtufa, truncata labii bafi exteriori inferti.

Mandibula exferta, cornea, arcuata, acuta, edentula.

Maxilla recta, porrecta, cylindrica inme. dio vnidentata, apice obtufa, rotundata.

Labium membranaceum, elongatum faepe longitudine fere palporum pofteriorum, rotundatum, bifidum: laciniis ae. qualibus, diftantibus, rotundatis, inte. gris.

Anternde elongatae, fetaceae, articulis cy. lindricis, fubaequalibus; primo incrafo fato.

Metamorphofis ignota.

Victus laruae ignotus, imaginis e floribus plantarum. 
47. CALOPVS. Cerambyx. Linn.

Os maxillis palpisque.

Palpi quatuor, inaequales.

anteriores longiores, clauati, quadriarticulati : articulo fecundo longiori, vltimo craffiori, truncato adhaerentes maxillae.

pofteriores filiformes, triarticulati : articulis aequalibus labii bafi exteriori inferti.

Mandibula breuis, cornea, arcuata, acuta, inermis.

Maxilla porrecta, fubarcuata, bifida: laciniis obtufis; exteriore fublongiore, tenuiore.

Labium porrectum, membranaceum, ad medium bifidum: laciniis aequalibus, rotundatis, diftantibus.

Antennae filiformes: articulis compreffis apice altero latere prominulis; primo craffiori, clauato.

Metamorphofis adhuc omnino latet.

victus \& laruae \& imaginis ignotus.

48. RHA- 


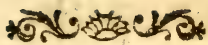

48. RHAGIV M. Cerambyx. Linn.

Stenocorus Geoff.

Os maxillis palpisque.

Palpi quatuor, inaequales, capitati.

anteriores longiores, quadriarticulati: articulis aequalibus, vitimo capitato, truncato adhaerentes maxillae.

pofteriores triarticulati: articulo vltimo clauato, truncato labii bafi inferti.

Mandibula breuis, cornea, arcuata, acuta, inermis.

Maxilla membranacea, obtufa, adinfertionem palporum vnidentata.

Labium breue, membranaceum, bifidum: laciniis aequalibus, diftantibus, rotun. datis.

Antennae elongatae, fetaceae: articulis cy. lindricis; primo craffiori clauato, vl. timo fetaceo.

Larua hexapoda, annulata, fubpilofa.

Puppa intra globum quiefcens.

Victus \& laruae \& imaginis e truncis arbo. rum putrefcentibus. 
49. SAPERDA. Cerambyx Linn. Leptura Geoff.

Os maxillis palpisque.

Balpi quatuor, fubaequales, filiformes.

enteriores quadriarticulati : articulis ro. tundatis; fecundo quarroque longio. ribus adhaerentes maxillae.

pofferiores triarticulati: articulis fubaequalibus, labii medio exteriori inferti.

Mandibula arcuata, cornea, acuta, eden. tula.

Maxilla membranacea, cylindrica, bifida: laciniis inaequalibus; exteriore paullo breuiore craffiore.

Labium membranaceum, adinfertionem palporum paullo coarctatum, apice rotundatum, dilatatum, truncatum.

Antemnae elongatae, fetaceae: articulis cylindricis; primo clauato, craffiore.

Metaenorphofis ignota.

Victus laruae e trunco putrefcente ar. borum, imaginis e foliis. 


\section{*⿻一𠃋十}

50. CALLIDIVM. Cerambyx. Linn. Leptura Linn. Geoff.

Os maxillis palpisque.

Palpi quatuor, aequales, clauati: articulo vltimo cratfiori, truncato.

anteriores quadriarticulati: articulis fubmoniliformibus adhaerentes maxil. lae dorfo.

pnferiores triarticulati: articulis vltimo excepto aequalibus, labii bafi exteriori inferti.

Mandibula breuis, cornea, arcuata, acuta, fubdenticulata.

Maxilla cylindrica, mernbranacea, recta, apice ouata, acuta, bifida: laciniis oblique truncatis, fubaequalibus; exteriore paullo maiore.

Labium porrectum, membranaceum, bifidum : laciniis aequalibus, tenuifimis, acutis, diftantibus.

Antemac elongatae, fetaceae: articulis cylindricis, fubaequalibus; primo crafio. re clauato.

Metamorplofis ignota.

Vicius laruae e trunco arborum putrefcente, imaginis e foliis plantarum. 


\section{I. DONA CIA. Leptura Linn. Stenocorus Geoff.}

Os maxillis palpisque.

Palpi quatuor, aequales, filiformes.

anteriores quadriarticulati: articulis aequalibus adhaerentes maxillae dorfo.

pofferiores triarticulati: articulis aequa. libus labii medio laterali inferti.

Mandibula breuis, fornicata, cornea, den. tata, apice fiffa.

Maxilla cylindrica, recta, inmedio vni. dentata.

Lakium elongatum, membranaceum, cy. lindricum, apice truncatum, integrum.

Antennae porrectae, fetaceae: articulis cylindricis, fubaequalibus; primo craffio. re, fecundo breviffimo.

Metamorphofis ignota, larua forfitan aquatica.

Viđtus laruae ignotus, imaginis e foliis plantarum. 
52. LEPTVRA. Linn. Stenocorus Geoff. Necydalis Linn.

Os maxillis palpisque.

Palpi quatuor, inaequales, filiformes.

anteriores quadriarticulati: articulis aequalibus; vitimo truncato adhaerentes maxillae dorfo.

pofteriores triarticulati : articulis aequa. libus labii bafi inferti.

Mandibula cornea, fubarcuata, acuta, edentula.

Mexilla recta, cylindrica, membranacea, inmedio vnidentata, apice fetofa.

Labium elongarum, membranaceum, fubcylindricum, porrectum, bifidum: laciniis aequalibus, diftantibus, rotundatis.

Antennac elongarae, fetaceae: articulis cylindricis, aequalibus; primo craf. fiore, clauato.

\section{Metamorphofis ignota.}

Vičus laruae forte e ligno putrefcente, imaginis e foliis plantarum.

Obf. Leptura $18-2 \mathrm{r}$. elytris abbreuiatis differunt, attamen certe huius generis.

$$
\mathrm{D}_{4} \text { 53. LAM. }
$$




\section{Noverar}

\section{LAMPYRIS. Linn. Geoff.}

Os maxillis palpisque.

Pslpi quatuor, inaequales.

enteriores longiores, fubclauati, quadriarticulati : articulo vltimo craffiori, fubulato, acuto adhaerentes maxil. lae dorfo.

pofferiores fubclatuati, triarticulati: articulo vltimo, craffiori,fubulato; acuto, labii apici inferti.

Mandibula cornea, arcuata, tenuiffima, acuita, edentula.

Maxilla breuis, membranacea, cylindrica, bifida! laciniis inaequalibus; exteriare maioie, rotundata.

Labium breue, corneum, cylindricum, gibbum, integerrimum.

Antentace filiformes: articulis aequalibus, obconicis; vitima cylindrico.

Metamorpinofis haud rite cognita.

Wictus \& lartae \& imaginis adhuc latent.

06f. I. Antennae interdum articulis brenio. ribus fere moniliformibus, interdum ferratis aut pectinatis.

Obj.II. Faemina in omnibus fpeciebus aptera. 
54. PYROCHROA. Geoff. Lampyris Linn. Geoff.

Os maxillis palpisque.

Palpi quatuor, inaequales, fubfiliformes. anteriores paullo longiores, quadriarticulati : articulis aequalibus; vltimo flexo fubcraffiori, adhaerentes ma. xillae dorfo.

pofferiores bafi coeuntes, triarticulati : articulis aequalibus, labii apici inferti.

Mandibula breuis, cornea, arcuata, acura, edentula.

Maxilla membranacea, fubcylindrica, integra, pilofa, apice acuta.

Labium porrectum, corneum, lineare, in. teg errimum, bafi compreffum.

Antennae filiformes, articulis breuibus apice interiori porrectis, acuminatis; primo longiori craftiori.

Metamorphofis mihi later.

Victus \& laruae \& imaginis omnino latet.

Obf. Pyrochroa 5 -8. habitu a reliquis diffe. runt \& forte noutum genus ore porre. Eo, cylindrico conftitunt mihi haud rite examinatae. 
55. LYMEXYLON. Cantharis Linn.

Os maxillis palpisque.

Palpi quatuor, inaequales, extrorfum craffiores.

anteriores longiores, exferti, dependentes, fentim crafiores, quadriarticulari : articulo vitimo longiore cylindrico adhaerentes maxillae dorfo.

pofteriores breues, triarticulati, obtufirfimi labii medio laterali inferti.

Mandibula breuis, cornea, fubrecta, inermis.

Maxilla breviffima, membranacea, craffa, bifida: laciniis inaequalibus; exteriore paullo longiore, rotundata.

Labium porrectum, membranaceum, lineare, tenuilfimum, apice rotundatum, concauum, fubemarginatum.

Antenrac breves, incuruae, moniliformes: articulis breuibus; apicibus vtrinque prominulis.

Metamorpbofis latet.

Vičtus \& laruae \& imaginis e ligno recenti. 


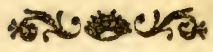 56. CVCVIVS.}

Os maxillis palpisque.

$P$ ulpi quatuor, breues, aequales.

anteriores triarticulati: articulo primo obconico, fecundo tertioque bre. uioribus, obtufis, truncatis adhaeren. tes maxillae dorfo.

pofteriores biarticulati: articulo primo obconico, vltimo craffiori, obrufo, truncato labii bafi anteriori inferti.

Mandibula cornea, arcuata, bafi incrafta. ta, apice acuminata, edentula.

Maxilla breuis, membranacea, bifida: la ciniis inaequalibus; exteriore maiore, rotundata, interiore acuta.

Labium breue, membranaceum, bifidum: Jaciniis linearibus, obtufis, diftantibus, palpis pofterioribus tectis.

Antennae moniliformes: articulis vndecim, breuibus, pilofis; vltimo acuto.

Metamorphofis \& Victus omnino adhuc latent.

Obf. Palatum vtrinque unidentatum. 
57. CANTHARIS. Linn. Cicindela Geoff. Necydalis Geoff.

Os maxillis palpisque.

Palpi quatuor, inaequales, fectiriformes. anteriores longiores, quadriarticulati: articulis aequalibus; vltino claua dila. tata, acuminata adhaerentes maxillae.

pofteriores triarticulati: articulis aequali. bus; vltimo clana dilatata, acuminata labii apici inferti.

Mandibula porrecta, exferta, cornea, arcuata, acutifima, edentula.

Maxilla breuis, cylindrica, ohlique truncata, bifida: lacinilis aequalibus, filiformibus, obtufis.

Labium breue, membranaccum, cylindricum, truncatum, integerrimum.

Antennac filiformes: articulis cylindricis, aequalibus; fecundo bretiiftimo.

Metamorphofis ignota.

Victus laruae later, imaginis e rapina alio. rum infectorum. 
58. MALACHIVS. Cantharis Linn. Cicindela Geoff.

Os maxillis palpisque.

Palpi quatuor, inaequales, filiformes.

anteriores paullo longiores, quadriarticulati : articulis aequalibus; vltimo fetaceo adhaerentes maxillae dorfo. pefferiores triarticulati: articulis aequalibus; vltimo fetaceo labii medio in. ferti.

Mandibula cornea, arcuata, acuta, integra, edentula.

Maxilla cylindrica, fubincurua, obtufa, membranacea, in medio acure dentata.

Labium porrectum, cylindricum, mem. branaceum, apice rotundatum, integrum.

Antennae filiformes: articulis aequalibus, cylindricis; primo fubcraffiori, vltimo ouato.

Metamorpbofis ignota.

Victus laruae latet, imaginis e rapina in. fectorum.

Obf. ad bafin abdominis \& thoracis vtrinque tentacula duo exferta bafi connata gerunt. 
59. NECYDAI.IS. Linn. Cantharis Linn. Geoff. Cicindela Geoff. Leptura Geoff.

Os maxillis palpisque.

Palpi quatuor, inaequales, fubfiliformes. antcriores quadriarticulati: articulis ae. qualibus; vitimo fubcraffiori adhaerentes maxillae dorfo.

poferiores triarticulati: articulis aequalibus; vltimo fubcraffiori, truncato Jabii medio inferti.

Mandibula cornea, arcuata, acuta, edentula. Maxilla porrecta, cylindrica, membrana. cea, ad infertionem paiporum vniden. tata, acutiufcula.

Labium porrectum, membranaceum, cylindricum, ad infertionem palpoium coarctatum, apice late emarginatum.

Antenna filiformes: articulis aequalibus, cylindricis; primo craffiori.

Metamorphofis \& Victus adhuc omnino la. tent. 
60. ElATER. Linn. Geoff.

Os maxillis palpisque.

Palpi quatuor, inaecuales, fecuriformes.

anteriores quadriarticulati: articulis fub. aequalibus; virimo claua dilarata,acuminata adhaerentes maxillae dorfo.

pofteriores breuiores, triarticulati : articulis aequalibus; vltimo cluna dilatata, acuta labii medio inferti.

Mundibula cornea, arcuata, inermis, apice fiffa.

Maxilla cylindrica, membranacea, in medio vnidentata, apice obrufa, fetola.

Labium porrêum, membranaceum, apice fubdilatarum, bifidum, laciniis aequalibus, truncatis.

Antennae filiformes: articulis breuibus, faepius ferratis; primo craffiori.

Metamorpbofis \& Victus omnino adhuc la. tent. 
64

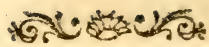

\section{I. BVPRES TIS. Linn. Cucujus Geoff.}

Os maxillis palpisque.

Palpi quatuor, inaequales, filiformes arti. culo vltimo obtufo truncato.

anteriores longiores, quadriarticulati: atticulis fubaequalibus adhaerentes ma. xillae dorfo.

pofferiores triarticulati, labii apici laterali inferti.

Mandibula breuis, cornea, arcuata, acu. ta, edentula.

Maxilla breuis, cylindrica, membrana. cea, medio vnidentata, apice rorunda. ta, obtufiffima.

Labium porredum, membranaceum, "cy. lindricum, inter palpos pofteriores acuminatum, integrum.

Antennac breues, filiformes, ferracae: articulis aequalibus, primo crafliori, viti. mo ouato, obrufo.

Metamorpbofis \& Victus \& larmae \& imaginis latent. 
62. CICINDELA. Linn. Bupreftis. Geoff. Os maxillis palpisque.

Palpi fex fubaequales, filiformes.

anteriores paullo breuiores, biarticulati: articulis aequalibus, longiffmis adhaerentes maxillae dorfo.

medii quadriarticulati: articulo primo tertioque breuifimis adnati anterio. rum bafi.

pofferiores multiarticulati articulis breuifimis, rotundatis, pilofis, vitimo longiori, obconico, nudo labii me. dio interiori inferti.

Mandibula elongara, exferta, cornea, arcuata, acuta, multidentata.

Maxilla recta, cornea, rigida, ciliata, apice incurua, acuta.

Labium breue, corneum, apice rridenta. tum, dentibus elongatis, rigidis, acutis.

Anternac elongatae, fetaceae: articulis cylindricis fubaequalibus excepto fecundo breuiffimo.

Metamorphojis latet.

Viftus laruae ignotus, imaginis e rapina laruarum. 


\section{ELA PHR VS. Cicindela. Iinn. Bupreftis Geoff.}

Os maxillis palpisque.

Palpi fex fubaequales, filiformes.

anteriores paullo breuiores, biarticulati: articulis aequalibus maxillae dorfo incumbentes.

medii longiores, quadriarticulati : articulo primo tertioque breuiffimis anteriorum bafi adnati.

pofferiores triarticulati : articulis aequalibus labii bafi laterali inferti.

Mandibula breuis, craffa, cornea, acuta in medio vnidentata.

Maxilla cornea, arcuata, intus ciliata, acuta, integra.

Labium breue, membranaceum, cylindricum, apice acuminatum.

Antennae fubferaceae: articulis breuibus, aequalibus, fubcylindricis primo craffiori.

Metamorphogis latet.

Victus laruae ignotus, imaginis e rapina infectorum.

06\% Differt manifefte a Cicindelis ipfa antennarum ftructura omnino alia. 


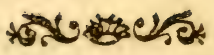

64. HYDROPHILVS. Geoff. Dyti-

fcus Linn.

Os maxillis palpisque.

Paipi quatuor, inaequales, filiformes.

anteriores longiores, quadriarticulati: articulo primo breuiffimo adhaeren. tes maxillae dorfo.

pofteriores triarticulati: articulis aequali. bus labii apici inferti.

Mandibula cornea, arcuata, inermis, acu. minata.

Maxilla breuis, nembranacea, bifida: la. ciniis inaequalibus; exteriore maiore rotundata.

Labium elongatum, corneum, rotunda. tum, fubemarginatum.

Antennae breues, perfoliatae: articulo primo craffiori longiori, fequentibus breuifimis vix diftinctis, penultimis duobus craffioribus perfoliatis latere interiori acuminatis, vltime ouato obtuío.

Lavua hexapoda, natatoriis vtrinque feptem, cauda biappendiculata.

Puppa quiefcens, ouata, pilofa, caudd femilunari.

Victus \& laruae \& imaginis e rapina pifcium, infectorum.

$$
\mathrm{E} \approx 6 \% \mathrm{DY} \text {. }
$$


68

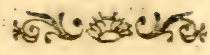

\section{DYTISCVS. Linn. Geoft,}

Os maxillis palpisque.

Palpi fex inaequales, filiformes.

anteriores breuiores, biarticulati: articulis aequalibus, vltimo acuto maxillae dorfo incumbentes.

mediilongiores, quadriarticulati: articua lis aequalibus bafi anteriorum ad. haerentes.

pofteriores triarticulati, labii medio in. ferti.

Mandibula exferta, cornea, arcuata, acu. ta, edentula.

Maxilla cornea, ciliata, integra, acutif. fima.

Labiun elongatum, corneum, latum, truncatum, integerrimum.

Antenuae porrestae, feraceae: articulis fubaequalibus, cylindricis, vltimis tenuioribus.

Larus hexapoda, vltimo abdominis articulo conico faepius bicaudato maxillis porrectis, arcuatis, inermibus.

Puppa quiefcens, moliis, ouata intra gle. bam.

iiclus \& laruae \& imaginis e rapina infectorum, laruarum pífiumgue. 


\section{-}

65. GYRINVS. Linn. Geoff.

Os maxillis palpisque.

Palpi quatuor, aequales, filiformes.

anteriores vir longiores, quadriarticu. lati: articulis breuibus rotundatis, vltimis duobus fubcraffioribus adhaerentes maxillae bafl.

poferiares cylindrici, triarticulati : articulis aegualibus labii medio interiori inferti.

Mandibula cornea, craffa, arcuata, apice bidentata.

Maxilla porrecta, cornea, arcuata medio vnidentata, apice acutiffima.

Labim porrectum, corneum, rotundatum profunde emarginatuni: laciniis rotundatis.

Antenna breuifimae, cylindricae, filiformes, rigidae: articulis breuiffimis vix diftinetis.

Iarua hexapoda, filiformis: natatoriis $\mathrm{v}$ trinque nouem, cauda quadrifida.

Puppa ignota.

Vietus \& laruae \& imaginis e rapina reliquorum infectorum.

$$
\mathrm{E}_{3} \quad 6 \% \mathrm{CA} \text {. }
$$


67. CA R A B V S. Linn. Bupreltis Geof: Os maxillis palpisque.

Palpi fex, inaequales: articulo vltimo obiu. fo, truncato.

anteriores breuiores, filiformes, biarticulali, obtufi, truncati adhaerentes ma. xillae dorfo.

medii longiores, quadriarticulati : articulo primo breuiffimo, reliquis obconicis bafi anteriorum adnati.

pofteriores triarticulati, bafi connati: articulo primo breuiffino, fecundo cy. lindrico longiori, rertio obconico fub labii apice inferti,

Mandibula cornea, arcuata, acuta, integra. Maxilla integerrima, cornea, cylindrica, intus ciliara apice arcuata, acutiffima. Labium porrectum, membranaceum, cy. lindricum, truncatum, integerrimum. Antemne filiformes: articulis elongatis, aequalibus, obconicis vltime cylinảri. co obtufo.

Larua hexapoda, depreffa, coriacea, maxillis exfertis, arcuatis.

Puppa ignota.

Victus laruac e quisquiliis, ligno putre. fcente, imaginis e rapina reliquorum infectorum, in primis laruarum.

Obf. I. In Carabis maioribus palatum elongarum tridentatum, labium tegens. Obf. II. C. Ipinibarbis mandibula bafi dilatata, maxillaeque dorfo fpinis ciliato. 68. 


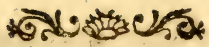

68. SCARITES. Tenebrio. Linn.

Os maxillis palpisque.

Palpi fex, fubaequales, filiformes.

anteriores breuiores, biarticulari: articu-

lis aequalibus, cylindricis adhaerentes maxillae dorfo.

mediilongiores, triarticulati: articulo lon. giori, fetaceo, acuto bafi anteriorum adnati.

poferiores triarticulati: articulis aequalibus labii medio laterali inferti.

Mandibula cornea, arcuata valida, acuta, intus denrata.

Maxilla cornea, arcuata, intus ciliata, acuminata.

Labium corneum, porrectum, cylindricum, apice tridentatum, dentibus ae. qualibus acutis.

Antennas moniliformes, articulo primo longiori fecundoque obconicis, reliquis breuioribus orbiculatis.

Metamorphofis \& victus \& laruae \& imaginis omnino adhuc latent.

Obf. S. clauicornis antennas extrorfum craffiores haber, attamen huius generis.

$$
\text { E } 4 \text { 69. SE. }
$$


72

\section{स.}

\section{SEPIDIVM.}

Os maxillis palpisque.

Palpi quatuor, inaequales, filiformes.

anteriores longiores, quadriarticulati: articulis cylindricis, fecundo longiori, vltimo obtulo adhaerentes maxillae dorfo.

pofteriores breuiores, triarticulati : articu. lis aequalibus, labii bafi laterali inferti.

Mandibula breuis, cornea, valida, arcuata, apice fiffa.

Maxilla breuis, cylindrica, membranacea, apice obtufa, rotundata, ciliata, medio vnidentata.

Labium breue, corneum, cylindricum, latum vsque adbafin fere emarg!natum.

Antenstac filiformes: articulo fecundo longiori, reliquis breuibus cylindricis, vl. timo ouato, acuto.

Metamorphofis \& victus \& laruae \& imaginis omnino adhuc latent.

obf. Genus Pimeliis affine, at habitu \& cha. ractere omnino difinetum. 
7o. PIMELIA. Tenebrio Linn. Geoff.

Os maxillis palpisque.

Palpi quatuor, inaequales, filiformes.

anteriores multo longiores, quadriarticulati : articulo fecundo longiori, vltimo fubglobofo, obtufo adhaeren. tes maxillae dorfo.

pofferiores breuiores, triarticulati: arti. culis aequalibus labii bafi laterali inferti.

Mandibula magna, cornea, gibba, craffa, compreffa, denticulata.

Maxilla cylindrica, membranacea, medio vnidentata apice obtufa, rotundata.

Labium porrectum, corneum, rotundatum, apice truncatum, fubemargina. tum, pilofum.

Antennae filiformes, apice moniliformes: articulo fecundo longifimo, vltimis quatuor breuioribus, moniliformibus。 Metamorploges \& Victus omnino adhuc latent.

$$
\text { Es 7r. SCAV - }
$$


Os maxillis palpisque.

Palpi quatuor inaequales, filiformes.

anteriores longiores, quadriarticulati : articulis cylindricis, fecundo longiori adhaerentes maxillae dorfo.

pofteriores breuiores, triarticulati: articulis cylindricis, breuiffimis labii bafi laterali inferti.

Mardibula breuis, cornea, arcuata, acuta, inermis.

Maxilla recta, membranacea, medio vridentata, apice dilatata, rotundata.

Labium corneum, rotundatum, latum, truncatum, integerrimum.

Antenrac moniliformes: articulis inferioribus longioribus (inprimis fecundo) obconicis, reliquis aequalibus, breuibus, moniliformibus.

Metamorphofis \& Victus \& laruae \& imaginis omnino adhuc latent. 
72 BLAPS. Tenebrio Linn. Geoff.

Os maxillis palpisque.

Palpi quatuor, inaequales, clauati: claua truncata, obtufa.

anteriores longiores, quadriarticulati: articulo fecundo longiori, tertio orbiculato adhaerentes maxillae dorfo.

pofteriores triarticulati: articulis aequalibus labii bafi laterali inferti.

Mandibula cornea, arcuata, integra, acuta. Maxilla recta, porrecta, bifida: laciniis inaequalibus; exteriore maiori, obrufa, rotundata.

Labium breue, membranaceum, rotunda. tum apice fiffum.

Antennae filiformes apice moniliformes: articulo fecundo longiori obconico, vltimis quatuor orbiculatis moniliformibus.

Metamorphofis \& Victus omnino latent. 
73. TENEBRIO. Linn Geoff. Platycerus Geoff.

Os maxillis palpisque.

Palpi quatuor, inaequales.

anteriores longiores, quadriarticulati: articulis fubaegualibus, vlitimo obtufo, truncato craffiori adhaerentes maxillae dorfo.

pofferiores triarticulati, filiformes: articulis aequalibus labii medio laterali inferti.

Mondibula cornea, arcuata, acuta, integerrima.

Maxilla recta, cylindrica, membranacea, bifda: laciniis inaequalibus; exteriori maiori obtufa, interiori acuta.

Labium porrectum, corneum, cylindri. cum, medio parum coarctatum apice truncatum, integerrimum.

Antenne moniliformes: articulis fubaequalibus excepto primo longiori obconico.

Larus hexapoda, annulata, mollis, cauda ftylis duobus brevibus, incuruis.

Puppa quiefcens, onara, pilofa, cauda ftylis duobus, breuibus, filiformibus. BiEtus \& laruae \& imaginis e quisquilis, farina, aliisque. 


\section{*is.}

\section{HELOPS. Tenebrio Linn. Geoff.}

Os maxillis palpisque.

Palpi quatuor, inaequales.

anteriores porrecti, longiores, fecuriformes, guadriarticulati: articulis aequalibus, vltimo clauato fecuriformi adhaerentes maxillae dorfo.

pofseriores breuiores, clauati: claua capitata, obtufa, triarticulati labii medio laterali inferti.

Mandibula cornea, arcuata, acuta, in medio vnidentata.

Maxilla cylindrica, membranacea, in me. dio vnidenrata, apice rotundata, obtufa.

Labium corneum, breue, rotundatum, in medio gibbum, apice truncatum, in. tegrum.

Antennae fubmoniliformes: articulis prio. ribus obconicis, fecundo longiori, vltimo ouato, obtufo.

Metamorphofis \& Victus \& laruae \& imaginis latent.

Obf. Helops yerratus palpis maioribus, reflexis, ferratis differt, attamen huius generis.

75. ERO. 


\section{मำ}

\section{ERODIVS.}

Os maxillis palpisque.

$P_{a l p i}$ quatuor, aequales, filiformes.

anteriores vix longiores, quadriarticulati: articulis aequalibus adhaerentes maxillae dorfo.

pofteriores triarticulati: articulo vltimo globofo fubcraffiori labii bafi lacerali inferti.

Mandibula cornea, arcuata, acuta, edentula. Maxilla cornea, recta, rigida, bifida: laciniis aequalibus, truncatis, obtufis, ciliatis.

Labium corneum, rotundatum, trunca. tum, emarginatum, ciliatum.

Antennae breues, moniliformes : articulis fubaequalibus, fecundo longiori, cylincirico.

Metamorplofis \& Vietus \& laruac \& imaginis ignoratur. 
76. MELOE. Linn. Geoff.

Os maxillis palpisque.

Palpi quatuor, inaequales, extrorfum fubcraffiores.

anteriores longiores: articulis aequalibus, vltime fubcraffiori, obtufo, truncato, adhaerentes maxillae dorfo.

pofteriores quadriarticulati : articulis minoribus, rotundatis labii medio inferti.

Mandibula exferta, cornea, arcuata, acuta, edentula.

Maxilla recta, membranacea, bifida: laciniis fubaequalibus, rotundatis, obtufis; exteriore paullo maiore.

Labium porrectum, corneum ad infertionem palporum coarctatum, apice rotundatum, emarginatum.

Antennae moniliformes: articulis breuibus, fubaequalibus, fecundo paullo longiori, vltimo fetaceo.

Larua hexapoda, filiformis lateribus fubpilofis.

Puppa quiefcens, mollis.

Vičtus \& laruae \& imaginis e foliis plantarum.

Obf. Antennarum articulus intermedius interdum dimidiatus an Varietas fexus? 
77. LYT TA. Meloe Linn. Cantharis Geoff,

os maxillis palpisque.

$p_{\text {alpi quatuor, inaequales. }}$

anteriores longiores, triarticulati: articulis aequalibus, vltimo fetaceo ad. haerentes maxillae dorfo.

pofteriores triarticulati: articulis aeçualibus, vltimo obtuío, truncato, cla. uato labii medio inferti.

Mandibula crafla, cornea, acuta, inermis. Maxilla recta, porrecta, merrbianacea, apice dilatata, rotundata, bifida: laciniis conniuentibus inaequalibus; exteriore maiore.

Labium elongatum, fubcorneum, cylindricum ad infertionem palporum co. arctatum, apice truncatum, integrum.

Antenna filiformes: articulis aequalibus, fubcylindricis; primo craffiori, fecundo breuiffimo vltimo, fetaceo.

Motamorphofis \& Victus omnino ignotus. 
78. MYLABRIS. Meloe Linn.

Os maxillis palpisque.

$P_{\text {alpi }}$ quaruor, inaequales, filiformes.

anteriores longiores, quadriarticulati: articulis fubaequalibus adhaerentes maxillae dorfo.

poferiores triarticulati : articulis aequalibus labii medio laterali inferti.

Manaibula crafta, breuis, cornea, acura, inermis.

Maxilla recta, portecta, cornea, com. preffa, apice oblique truncara, obrufa bifida: laciniis aequalibus, conniuentibus.

Zabium porrectum, membranaceum, cy. lindricum ad palporum infertionem coarctatum, apice emarginatum.

Autenrat moniliformes, extrorfum craffiores: articulis fubaequalibus fecundo breuiori,

Metamorphofis \& Victus omnino latent. 


\section{CEROCOMA. Geofr. Meloe Linn.}

os maxillis palpisque.

Palpi quatuor, acquales, filiformes.

anteriores gua trarticulati: articulis cy.

lindricis aegualibus adhaerentes ma. xillae dorfo.

poferiores vix breuiores, triarticulati: articulis cylindricis, aequalibus labii medio laterali inferti.

Mandiúnla conea, porrecta, arcuata apice acuminata, edentula latere interiori dilatata, membranacea.

Maxilla cylindrica, linearis, membranacea, apice acuminata, letora, integerrima.

Lalium cylindricum, membranaceum, elongatum ad palporun infortionem crartarum apice bifitum: lacinis aequalibus rotundatis.

Antenase monilifomes: articulis aequalibus, breubus; vltimo maiori, clatuato, comprefio.

Obf. In altero fexu tertius palporum anterio. rum articulus incrafratus, veficulofus.

06\% In altero fexu arriculi antennarum. 3.4 . 5.6. dilatati, foliacei, perfoliati. 
8o'. MOR DELLA. Linn. Geoff.

Os maxillis palpisque.

Palpi quatuor, inaequales.

anteriores longiores, porrecti, quadriarticulati: articulis aequalibus; vltimo valiori adhaerentes maxillae dorfo.

pofferiores breniores, filiformes, triar. ticulati: articulis aequalibus labii medio laterali inferti.

Mandibula cornea, arcuata, acuta, inermis. Maxilla membranacea, linearis, bifida: lacinis obtufis, inaegualibus; exteriore maiore.

Labium elongatum, membranaceum, lineare, inter palpos porrectum, dilatatum, rotundatum, bifidum: laciniis aequalibus, rotundatis.

Antemac fub moniliformes: articulo primo longiori, fecundo breviffimo globofo, vitimo ouato, acutiufculo.

Metamorpbofis ignota.

Victus laruae ignotus, imaginis e floribus plantarum, inprimis vmbellatarum. 
8r. STAPHYLIN VS. Linn. Geoff. Os maxillis palpisque.

Palpi quatuor, aequales, filiformes.

anteriores quadriarticulati: articulis ae: qualibus adhaerentes maxillae dorfo. poferiores vix breuiores, triarticulati: articulis aequalibus labii bafi exte. riori inferti.

Mandibula porrecta, exlerta, cornea, ar. cuata, acuta in medio dentibus validis armata.

Maxilla membranacea, recta, cylindrica, in medio obtufe dentata.

Labium membranaceum, fub palpis elongatum, trifidum: laciniis fubaequalibus; intermedia latiore apice rotunda. ta, fubemarginata, lateralibus paullo longioribus, diftantibus, acutis.

Antennae moniliformes: articulis fubaequa. libus, breuibus, apice truncatis, vltimo oliato, obtufo.

Metamorphofis ignota.

Victus \& laruae \& imaginis e cadaueribus \& rapina animalium. 
82. OXY PORVS. Staphylinus Linn.

Geoff.

Os maxillis palpisque.

Palpi quatuor, fubaequales.

sintcriores quadriarticulati: articulis aequalibus, filiformibus adhaerentes maxillae bafi.

pofferiores vix longiores, quadriarticulati: articulis aequalibus; vltimo cla. uato, fecuriformi labii bafi laterali inerti.

Mandibula exferta, cornea, arcuata, acuta, inermis.

Maxills membranacea, cylindrica, medio vnidentata, apice ouata, obtufa.

Labium elongatum, membranaceum, cylindricum, emarginatum cum mucrone. Antenuse moniliformes extrorfum fubcraffiores, articulis fubaequalious fubpilo. fis apice truncatis, vitimo ouato, acuto. Larua hexapoda, mollis, laevis poftice attenuata.

Puppa ignota.

Vittus \& laruae \& imaginis e Boletis, Agaricis alisque fungis.

$$
\text { F 3 83. PAE }
$$


86

मำ

\section{PAEDERVS. Staphylinus Linn. Geoff.}

Os maxillis palpisque.

Palpi quatuor, inaequales.

anterines porrecti, multo longiores, capitati, quadriarticulari : articulis aequalibus adhaerentes maxiliae dorfo.

pofteriores breues, triarticulati : articu. lis aequalibus, filiformibus labii apice laterali inferti.

Mandibula cornea, arcuata, acuta, inermis. Maxilla membranacea, cylindrica, fubarcuata, medio vridentata.

Labiun porrectum, membranaceum, cy. lindricum, apice rruncatum, obtufum, integrum.

Antennae moniliformes: ariculo primo longiori, cratfiori, reliquis aequalibus, orbiculatis, vltimo acutulculo.

Metamorplofis \& vieus \& laruae \& imagi. nis omnino adhuc larent. 


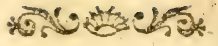 \\ Clafis II. \\ VLONATA.}

$O$ s maxillis palpisque.

$P_{a}{ }^{\prime} p i$ quatuor, veficulofi, porrecti, filifornes, obtufi : anteriores inidentes maxilae dorfo, pofteriores labio.

Clypeus horizontalis, membranaceus, rotundatus, os fuperne tegens.

Mandibulac duae, transuerfales, cornege, mobiles, dentatae, latera oris luperne includentes.

Maxillac duae, liberae, transuerfales, acutae \& fiepius dentatae ant fiffae latera oris inferne includentes.

Galea maxillam tegens, cylindrica, inarticulata, veficulofa, obtufa maxillae dorfo inferta.

Labium porrectum, liberum, membrana. ceum, femper apice fiftum.

Auterane inter oculos infertae. 
GENERA Claffis II.

84. FORFICVLA.

85. BLATTA.

86. MANTIS.

8\%. ACRYDIVM.

88. TRVXALIS.

89. ACHETA.

9o. LOCVSTA.

91. GKYLLVS. 


\section{FORFICVLA. Linn. Geoff.}

Os maxillis palpisque.

Palpi quatuor, inaequales, filiformes.

anteriores lonģiores, quadriarticulati: articulo primo, breuiffimo, reliquis aequalibus adhaerentes maxillae dorfo. poferiores multo breviores, triarticulati: articulo primo breuiffimo labii bafi inferti.

Mandibuls cornea, arcuara, acuta.

Maxilla cornea, arcuata, acuta, apice finta.

Galea maxillam tegens, cylindrica, obtufa longitudine maxillae.

Labium elongatum, porrectum, trifidum: laciniis inaequalibus, lateralibus, paul. lo longioribus, tenuioribus, cylindricis, obtufis; intermedia latiori, rotun. data, integra.

Antennac fetaceae: articulo primo longiori, craffiori, reliquis aequalibus, cy. lindricis.

Larua hexapoda, agilis, imagini fimillima, aptera.

Puppa hexapoda, agilis, irmagini fimillima alarum rudimentis.

Victus \& laruae \& imaginis e fructibus plantisque fucculentis.

$$
\text { F } 5 \text { 85.BLAT. }
$$


85. BLA T T A. Linn. Geoff.

Os maxillis palpisque.

Palpi quatuor, insequales, filiformes.

arteriores longiores, quinquearticulati: articulo primo fecundoque breuifimis, tertio longilimo adhaerentes maxillae dorfo.

pofferiores triarticulati: articulis aequalibus labii medio inferti.

Mandibula cornea, arcuata, acuta, inermis. Maxilla cornea, arcuata, acuta, apice fira. Galea maxillam tegens, cylindrica, obtufa, truncata, maxilla longior.

Labium elongatum, rotundatum, bifidum: laciniis aequalibus, rotundatis, apice filis; pinna exteriore traiore rotundata.

Antenua porrectae, feraceae: articulis innumeris, breuifimis, primo craffiori, ollato.

Lnrua hexapoda, agilis, currens, imagini fimillima, apiera.

Puppa hexapoda, agilis, imagini frmillima alarum rudimentis.

Viffus \& laruae \& imaginis e farina, radicibus plantarum, laccharo aliisque rebus culinaribus. 


\section{MANTIS. Linn. Geoff.}

Os riaxillis palpisque.

Palpi quatuor, fubaequales, filiformes.

anteriores vix longiores, quinquearticulati: articulo primo fecundoquebreuioribus achaerentes maxillae dorío. pofterinres triarticulati: articulo virimo fubulato labii medio laterali inferti.

Mandibula fubcornea, breuis, recta, acuminata, medio bidentata.

Maxill cornea, compreffa, ciliata, apice acutilima, integra.

Galea cylindrica, maxillis longior, apice rotundara, maxillam tegens.

Labium porrectum, membranaceum, bifidum: laciniis aequalibus, vsque ad medium fillis; pinnis aequalibus.

Antcnate fetaceae: articulis breuibus, primo longiori, reliquis aequalibus.

Larua hexapoda, currens, agilis, imagini fimillima, aptera.

Pupp hexapoda, agilis, currens, rudimentis alarum.

Victus \& irvae \& imiginis e rapina In. fectorum. 


\section{ACRYDIV M. Geoff. Gryllus Linn.}

Os maxillis palpisque.

Palpi quatuor, fubaequales, filiformes.

anteriores quadriarticulati: articulis ae. qualibus adhaerentes maxillae dorfo.

pofferiores triarticulati: articulis aequalibus labio medio laterali inferti.

Mindibula breuis, cornca, arcuata, acu. ta, apice dentata.

Maxilla cornea, arcuata, acuta, apice fiffa. Galea maxillam tegens, cylindrica, obtufa longitudine maxillae.

Zabium membranaceum, cylindricum, elongatum adinfertionem palporum coarctarum, apice fiffum.

Antennae filiformes, articulo primo longiori, crafliori; reliquis breuibus, acqualibus, vltimo fetaceo.

Laru hexapoda, aptera, agilis, currens, imagini fimillima.

Puppa hexapoda, agilis, imagini fimillima, folis alarum rudimentis. 
88. TRVXA LIS. Gryllus. Linn.

Os maxillis palpisque.

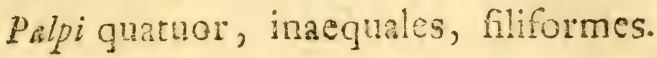

anteriores longiores, quinguearticulati: articulis aequalibus; cylindricis ad. haerentes maxillae dorfo.

pofteriores triarticulati : articulis aequalibus, cylindricis labii bafi laterali inferti.

Mandibula brenis, cornea, arcuata, acuta, edentula.

Maxilla cornea, fubarcuata, apice tridentata.

Galea maxillam tegens, lata, fornicata, longirudine maxillae.

Labium porrectum, membranaceum, ro. tundatum, bifidum: laciniis aequalibus, rotundatis, integris.

Antennse enfiformes, fubulatae: articulis. breuibus bafeos trigonoprismaticis, apice cylindricis.

Larua hexapoda, currens, agilis imagini fimillima, aptera.

Puppa hexapoda, agilis, currens, imagini fimillima rudimentis tantum alarum. Victus e rapina infectorum, inprimis dipterorum. 
89. ACHETA. Gryilus Linn. Geof. Os maxillis palpisque.

Palpi quatuor, inaequales, filiformes.

anteriores longiores, guinguearticulat: articulo vitimo brenillimo, velicu. lofo, reliquis aequaibus adhaerentes maxillae dorfo.

poferiores quadriarticulati: articulo vltimo breuiori labii bafi inferti.

Maxdibula cornea, porresta, incurua, apice transuerfe truncata, dentara.

Maxilla connea, arcuata, acuminata, api. ce fifía.

Galea maxillam tegens, cylindrica, ob. tura longitudine maxillae.

Labium elongatum, fubcylindricum, quadrifidum: lacinis aequalibus; exterioribus membranaceis, cylindricis, obturis; interioribus conneis, cylindricis apice fubulatis, a.uris.

Antennue fetaceae: articulis innumeris, breuilimis, aequalibus; primo cralfiori. Laru hexapoda, agilis, aptera, imagini fimillima.

Puppa hexapoda, agilis, imagini fimillima alarum rudimentis.

Victus e radicibus plantarum plantisque ipfis. 
90. LOCVSTA. Geoff. Gryllus Linn.

Os maxillis pulpisque.

Palpi quatnor inaequales: articulo vltimo, obtufo, truncato.

anteriores quadriarticulari : articulis fubaegualibus adhaerentes maxillae dorro.

pofferioves triarticulati: articulis aequalibus labii medio inferti.

Mundibula cornea, arcuata, acuta, inermis. Maxilla cornea, arcuata, acuta, tridentata.

Galea maxillam tegens, cylindrica, ob. tufa maxillae longitudine.

Lalium membranaceum, bafi cylindricum, apice rotundatum, bifidum: laciniis aconslibus; latere interiore fetafubulata inftruetis.

Antrina feraceae, porrectae: articulis innumeris, breuifimis, aequalibus primo craffiori longiori.

Larua hexapoda, aptera, agilis, imagini fimillima.

P'uppa hexapoda, agilis, imagini fimillima at rudimentis tantum alarum.

Victus e foliis plantarvm. 
9r. GRYLLVS. Linn. Acrydjum Geoff. Os maxillis palpisque.

Palpi quatuor, fubaequales, filiformes. anteriores paullo longiores, quinouearticulati: articulis aequalibus adhaerentes maxillae dorfo.

pojeriores quadriarticulati: articulis acqualibus labii medio inferti.

Mandibula craffa, cornea, reeta, obtufa, dentata.

Maxilla breuis, cornea, fubarcuata, acura, apice tridentata.

Galea maxillam tegens, cylindrica, fornicata, obtufa, vix maxilla longior.

Labium elongatum, membranaceum, ad infertionem palporum coarctatum, apice incraffatum, rocundatum, bifidum: laciniis aequalibus, rotundatis; conniuentibus, fornicatis.

Antenna filiformes: articulis vndecin cy. lindricis, aequalibus; primo craffiori breuiori.

Larua hexapoda, aptera, agilis, imagini fimillima.

Puppa hexapoda, agilis, imagini fimillima, rudimentis tantum alarum. Victus \& laruae \& imaginis e foliis plantarum. 


\section{क्तै परन \\ Clajfis III. \\ S Y N IS T A T A.}

$O$ s maxillis palpisque.

Palpi quatuor, porrecti, cornei: anterioribus infidentibus maxillae, pofterioribus labio.

Clypeus corneus, rotundatus, horizontalis, os fuperne tegens.

Mandibulac duae, transuerfales, corneae, mobiles, acutae, faepius dentatae, latera oris fuperne includentes.

Mandibulae nullae Ephemeris proprium.

Maxillae duae, transuerfales, membranaceae, cum labio bafi connatae, larera oris inferne includentes.

Maxillae faepius porrectae, elongatae, inflexae, lacinias exteriores linguae conftituentes.

Lalium porrectum, membranaceum, interdum inflexum, in lacinias linguae interiores elongatum.

Antenna inter oculos infertae. 
98

GENERA Claffis III.

92. MONOCVLVS. 1O7.ICHNEVMON.

93. ONISCVS. IO8. EVANIA.

94. LEPISMA. 109. SPHEX.

95. PODVRA. IIO. TIPHIA.

96. EPHEMERA, 1 I. SCOLIA.

97. SEMBLIS. II2. CHRYSIS.

98. PHRYGANEA. II 3. THYNNVS.

99. HEMEROBIVS. I 14 . LEVCOSPIS. 100. MYRMELEON I15. BEMBEX. 101. ASCALA- 116. VESPA.

\section{PHVS. II\%. CRABRO.}

102. PANORPA. II8. ANDRENA.

103. RAPHIDIA. II9. APIS.

104. CYNIPS. 120. NOMADA.

I05. TENTHRE- I2I. TERMES.

DO.

106. SIREX.
122. FORMICA.

123. MVTILLA. 


\section{MONOCVLVS. Linn. Geoff. Bi- noculus Geoff.}

Os maxillis palpisque.

Palpi quatuor, inaequales.

anteriores extrorfum craffiores, triarticulati : articulo primo breuiffimo, reliquis aequalibus, fubtus capitis tefta adhaerentes.

poferiores triarticulati: articulo vltimo concauo, auriformi maxillae inferti.

Mandibula cornea, craffa, gibba, fubglobofa, altero latere truncata, denticulata.

Maxila fubmembranacea, bifida: laciniis inaequalibus, exteriore breuiore.

Labium membranaceum, rotundatum, integrum, cum maxilla vltra medium connatum.

Antennae ramofae: ramis fetaceis; articulis innumeris, breuiffimis, aequalibus, primo longiori.

Larua \& Puppa imagini fimillimae,pedatae, currentes, agiles exuuias tantum deporint.

Victus e plantis aquaticis.

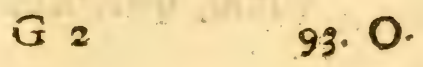


93. ONISCVS. Linn. Geoff. Afellus Geoff.

Os maxillis palpisque.

Palpi quatuor, inaequales, filiformes.

anteriores breuifimi, vix maxillae lon-

giores, cuins dorfo adhaerent.

pofteriores porrecti, longiores, quadriarticulati: articulis aequalibus, $\mathrm{cy}^{\mathrm{y}}$.

lindricis, vltimo fubulato labio inferti.

Mandibula breuis, craffa, apice acuta, dentata.

Maxilla recta, porrecta, membranacea, apice truncata, denticulata, connata cum labio.

Labium porrectum, membranaceum, rotundatum, bifidum: laciniis aequalibus, ouatis, obtufis.

Antennac interdum quatuor inaequales; anteriores breuiores, fubulatae, multiarticulatae, pofteriores longiores fetaceae, articulis duobus anterioribus longioribus, cylindricis, reliquis breuiffimis, innumeris, interdum duae filiformes, quinquearticulatae: articulo primo fecundoque breuioribus, tertio quartoque cylindricis, vltimo fetaceo, acuto.

Larua \& Puppa agiles, currentes, pedatae exuuias tantum deponunt.

Victus \& laruae \& imaginis e foliis plantarum, quisquiliis. 
94. LEPISMA. Linn. Forbicina Geoff.

Os maxillis palpisque.

Palpi quatuor, inaequales, filiformes.

antericres porredt, multo longiores;

quinguearticulati : articulis cylindri-

cis, fubaequalibus; vlrimo acumina.

to adhaerentes maxillae dorfo.

pofferiores breuiores, triarticulati: articulo vltimo, ouato, fubcraffiori labii medio inferti.

Mandibialu breuis, cornea, arcuata, acuta, inermis.

Maxilla porrecta, membranacea, cylin: drica, fubueficulofa, apice truncata, cum labio connata.

Labium membranaceum, porreftum, ro. tundatum, apice emarginatum.

Antenna fetaceae, porrectae: articulis innumeris, breuifimis, aequalibus; primo parum craftiori.

Larua \& Puppa hexapodae, agiles, currentes exuuias tantum deponunt.

Vitus \& laruae \& imaginis e faccharo, lig: no purrido, quisquiliis.
G 3
95. PO- 


\section{PODVRA. Linn. Geoff.}

Os maxillis palpisque.

Palpi quatuor, inaequales, fubclauati.

anteriores longiores, quinquearticulati: articulis fubaequalibus; exterioribus fenfim paullo craflioribus; fecundo breuiffimo adhacrentes maxillae dorfo.

pofteriores breues, triarticulati: articulis fubaequalibus; exterioribus fenfim craffioribus labii apici adnati.

Mandibula breuis, cornea, arcuata, acu. ta, inermis.

Maxilla porrecta, connata, membranacea, linearis, apice truncata, obtufa, integra.

Labium breue, membranaceum, rotundatum, bifidum: laciniis aequalibus, con. niventibus.

Antennae filiformes, quadriarticulatae: articulo primo breuiffimo, reliquis aequalibus, vltimo feraceo, acuro.

Loru \& Puppa hexapodae, agiles, cur. rentes, imagini fimillimac exuuias tantum deponunt.

Victus \& laruae \& puppae \& imaginis e foliis plantarum. 


\section{EPHEMERA. Linn. Geoff.}

Os maxillis palpisque.

Palpi quatuor, breuiltimi, fubaequales, filiformes.

anteriores quadriarticulati: articulis fub. acqualibus, cylindricis; fecundo paullo longiore adhaerentes maxillae dorfo.

pnfiriores triarticulati: articulis aequalibus, breuibus adhaerentes labii apici. Mandibula omino nulla.

Muxilla breuis, membranacea, cylindrica, obrufa, integra, cum labio arcte connata.

, Labium breue, membranaceum, cylindricum, apice rotundatum, integerrimum. Artonac breuifimae, fubulatae: articulo primo maiori, reliquis breuiffmis, vltimo acuto.

Larud aquatica, hexapoda, agilis, caudata, natatoris lateralibus, fenis.

Puppa hexapoda, agilis, caudata, natatoriis extrorfum craffionbus, alarum. que rudimentis.

Victus \& laruac \& puppae e foliis plantarum aquaticarum? imaginis omnino nullus.

Vira Imaginis vix viginti quatuor horas attingit ideogue cibo haud eget.

$$
\text { G } 4 \text { 9\% SEM- }
$$


97. SEMBLIS. Phryganea. Linn. Perla Geoff.

Os maxillis palpisque.

Palpi quatuor, elongati, fubaequales, filiformes.

anteriores vix longiores, quadriarticulati: articulo vltimo tenuiori, acuto adhaerentes maxillae lateri exteriori.

Poferiores triarticulati : articulis fubaequalibus; vltimo acuto labii medio laterali inferti.

Mandibula breuiffima, cornea, arcuata, acuta, inermis.

Maxilla breuis, membranacea, connata, fubarcuata, bifida: laciniis aequalibus, tenuifimis, acutis.

Labium porrectum, cylindricum, membranaceum, annulatum, integrum.

Antennae fetaceae: articulis plurimis, breuibus, primo paullo maiori.

Larus aquatica, hexapoda, caudata, natatoriis feptenis, filiformibus.

Puppa quiefcens, hexapoda, maxillis corneis, prominulis.

Viffus laruae e rapina aquatilium minimorum, imaginis ignotus. 
98. PHR YGANEA. Linn. Geoff.

Os maxillis palpisque.

Palpi quatuor, inaequales, approximati, porrecti, filiformes.

anteriores longiores, quinquearticulati: articulo fecundo tertioque longiffmis adhaerentes maxillae medio.

pofferiores breuiores, quadriarticulati: articulo primo plano, lateribus dilatatis, vltimo compreffo, latiore labii apici inferti.

Mandibula breuis, cornea, arcuata, acuta, inermis.

Maxilla reda, membranacea, acuta, integra, cum labio inferiori tota conna-

ta, vt vix appareat.

Labium porreetum, cylindricum, latum, membranaceum, apice emarginatum.

Antennac fetaceae: articulis innumeris, brenibus; primo paullo naaiori.

Larua aquatica, hexapoda, fubpilofa, vnguibus duobus caudalibus tentaculisque tribus erectis quarti fegmenti intra cucullum latitans.

Puppa quiefcens, folliculata, imagini fimillima, vnguiculis duobus caudalibus, brenibus.

Vitus laruae e rapina aquatilium minimorum, imaginis adhuc latet.
G 5
99. HE. 


\section{HEMER OBIV S. Linn. Geoff. Termes Linn.}

Os maxillis palpisque.

Palpi quatuor, porrecti, inaequales, filiformes.

anteriores longiores, quadriarticulati: articulo recundo longiori, reliquis aequalibus maxillae adhaerentes.

poftcriores triarticulati: articulo vltimo cylindrico, longiori labii medio la. terali inferti.

Mandibula breuis, cornea, craffa, apice acumirata.

Maxilla cylindrica, membranacea, recta, fifra: laciniis aequalibus, breuiffimis.

Labium corneum, porrectum, ad infertionem palporum coarctatum, apice rotundatum, fornicatum, integrum.

Antennae fetaceae: articulis plurimis, breuiflimis; primo maiore.

Laru hexapoda, ouata, pilofa.

Puppa folliculata, quiefcens.

Victus \& laruae \& imaginis e rapina Infecto. rum minimorum, inprimis Aphidum. 


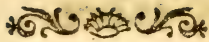

100. MYRMELEON. Linn. Formicaleo Geoff.

Os maxillis palpisque.

Palpi fex, inaequales, porrecti.

anteriores breuiores, filiformes, triarticulati : articulo fecundo longiffimo maxillae adhaerentes.

medii vix longiores, filiformes, quadri. articulati : articulo primo fecundoque longioribus anteriorum bafi in. ferti.

pofteriores longiflimi, 'quadriarticulati: articulo primo fecundogue breuill: mis, tertio quartoque longilimis, clauatis labii bafi laterali inferti.

Mandibula exferta, cornea, arcuata, acuta. Maxilla cornea, compreffa, bafi dilatata, vnidentata, apice acuta.

Labium porredtum, membranaceum, quadratum, truncatum, emarginatum. Antennac extrorfum craffiores : articulis plurimis, breuibus, primo maiori.

Larua hexapoda, ouata, pilofa, maxillis exfertis, dentatis.

Puppa incuruata, quiefcens intra glebam. Victus \& laruae \& imaginis e rapina formicarum aliorumque Infectorum. 
Ior. ASCAI.APHVS. Myrmeleon. Linn.

Os maxillis palpisque.

Palpi fex, fubaequales, filiformes.

anteriores paullo breuiores, triarticulati: articulo fecundo longilfimo ad. haerentes maxillae dorfo.

medii longiores, quadriarticulati : articulo fecundo longifrimo anteriorum bafi inferti.

pofteriores quadriarticulati : articulo primo \& quarto breuifimis labii bafi laterali inferti.

Mandibula cornea, arcuata, acuta, dentata. Maxilla cornea, fubarcuata, acuta, ciliata, integra.

Labium porrectum, corneum, rotundatum, integrum.

Antennae porredae, clauatae: articulis plurimis, breuibus; primo maiori, vltimis tribus craffioribus.

Metamorpbofis \& Victus adhuc nobis latent. 
102. PA NORPA. Linn. Geoff.

Os porrestum in roftrum cylindricum, cor-

neum, maxillis palpisque.

Palpi quatuor, fubaequales, filiformes.

anteriores vix longiores, quadriarticulati: articulis fubaequalibus adhae. rentes maxillae dorfo.

pofteriores breuiores, biarticulati: articulo primo crafliori, gibbo, labii apici inferti.

Mandibula porrecta, cornea, arcuata, acuta, edentula.

Maxilla cornea, porredta, apice truncata, bifida: laciniis fubaequalibus, cylindricis; exteriore tenuiore integra, interiore apice bidentata.

Labium elongatum, porrectum, corneum, fornicatum, rotundatum, integrum, totum os obtegens.

Antennae filiformes : articulis plurimis, breuiflimis; primo maiori.

Metamorphofis ignota, larua forfan aquatica. Victus laruae ignotus, imaginis e rapina reliquorum Infestorum. 
I03. RAPHIDIA. Linn. Geoff.

Os maxiilis palpisque.

Palpi quatuor, breuiffimi, fubaequales, filiformes.

antcriores paullo longiores, guadriarticulati: articulis aequalibus adhaerentes maxillae dorfo.

poferiores triartículati: articulis aequalibus labii apici inferti.

Mardibula cornea, compreffa, arcuata, acuta, dentata.

Maxilla breuis, cornea, cylindrica, ob. tufa, vix vnidentata, integra.

Labium breue, corneum, rotundatum, integrum, apice palpigerum.

Antennae porrectae, filiformes: articulis fubaequalibus; primo paullo maiori.

Larua hexapoda, agilis imagini fimillima at aptera.

Puppa ignota.

ViEtus \& laruae \& imaginis e rapina reliquorum Infectorum. 
r04. CYNIPS. Linn. Geoff. Diplolepis Geoff.

Os maxillis palpisque.

Palpi quatuor, breues, inaequales, capitati.

antcriores longiores, quinquearticulati: articulis cylindricis, fubaequalibus; vltimo rotundato, craffiori adhae. rentes maxillae dorfo.

pofteriores quadriarticulati: articulis cy. lindricis, aequalibus; vltimo rotundato, craffiori labii apici inferti.

Mandibula cornea, arcuata, apice fiffa.

Maxilla breuis, membranacea, vnidentata, cum labio arkte connata.

Labium breue, corneum, cylindricum, integrum.

Antennac porrectae, filiformes, multiarticulatae: articulis fubaequalibus; primo maiore.

Larua apoda, mollis, cylindrica, incurua intia gallam plantarum.

Puppa quiefcens, imagini fimillima, rıdimentis tantum alarum.

Bicfus e fucco plantarum e vulnere ftillan. te in Gallam excrefcente. 
105. TENTHRED O. Linn. Geoff. Crabro Geoff.

Os maxillis palpisque.

Palpi quatuor, inaequales, filiformes.

anteriores porrecti, multo longiores,

fex articulati: articulis fubaequalibus adhaerentes maxillae dorfo.

poferiores triarticulati : articulis accualibus labii medio inferti labium in. cludentes.

Mandibula exferta, cornea, arcuata, acu. ta, intus dentata.

Maxilla rećta, porrecta, compreffa, ad infertionem palporum vnidentata, apice obtufa.

Labium porrectum, cylindricum, membranaceum, ad infertionem palporum coarctatum, apice rotundatum, dilaratum, trifidum: laciniis fubaequalibus; intermedia tenuiore, obtufiore. Anienna clauatae, filiformes, feraceae. Larua cylindrica, mollis, pedibus fedecim vsque aduiginti.

Puppa quiefcens, imagini fimillima, rudimentis tantum alarum.

ivictus e foliis plantarum.

Crabro Geoff. differt labio longiori magis porrecto, antennisque clauatis, attamen huius generis. 
t05. SIREX. Linn. Vroceros Geoff.

Os maxillis palpisque.

Palpi quatuor, inaequales.

anteriores breniffimi, fliformes, biar. ticulati: articulis aequalibus adhae. rentes maxillae dorfo.

pofferiores porrecti, longiores, extrorfum craffiores, pilofi, quadriarticulati: articulis fubaequalibus labii apici inferri.

Mandibula breuis, craffa, cornea, apice truncata, denticulata.

Maxilla breuis, incurua, acuminata, membranacea, ciliata, cylindrica, integra. Labium breue, membranaceum, integrum. Antcnuae filiformes: articulis plurimis, breuibus, aequalibus, primo vix lon. giori.

Lmma hexapoda, mollis, cylindrica, ca. pite rotundato.

Puppa folliculata, quief́ens, imagini fimillima, alarum tantum rudimentis.

Victus laruae ex arborum ligno; imaginis e florum nectare. 
107. I CHNEV MON. Linn. Geoff. Mutilla Linn.

Os maxillis palpisque.

Palpi quatuor, porrecti, inaequales, filiformes.

antcriores longiores, fexarticulati : articulo fecundo obconico, tertio fubdilatato adhaerentes maxillae dorfo. pofteriores breuiores, quinquearticulati: articulo vltimo longiffimo, cylindrico labii medio larerali inferti.

Mandibula cornea, arcuata, acuta, inermis. Maxilla recta, membranacea, bifida, ad infertionem palporum vnidentata, apice rotundata, dilatata, ciliata.

Labium porrectun, corneum, cylindricum, gibbum, carinatum, apice membra. naceum, rotundatum, emarginatum. Antenna fetaceae: articulis plurimis, bre. uilfimis, aequalibus; primo maiori. Larua apoda, mollis, cylincirica. Puppa mox nuda, mox folliculata, quie. fcens, imagini fimillima rudimentis for lis alarum.

Vietus laruae e fucco animalium, intra corpora eorum habitantis, imaginis e ne. ctare florum.

Ich. luteus differt mandibula fornicata, iner. $\mathrm{mi}$, apice fifla. 
I08. EVANIA. Sphex Linn.

Os maxillis palpisque.

Palpi quatuor, inaequales.

anteriores longiores, porrecti, filiformes, fexarticulati: articulo vltimo longiori adhaerentes maxillae dorfo. poferiores incurui, quadriarticulati: articulo tertio craffiori, quadrato labii medio inferti.

Mandibula breuis, cornea, incurua, acuta, vnidentata.

Maxilla breuis, membranacea, compreffa, apice rotundata, integra.

Labium breue, corneum, conicum, apice membranaceum, acutum, integerrimum.

Antennae filiformes: articulis plurimis, cylindricis, aequalibus; primo maiori, vltimo acuto.

Metamorphofis \& Victus \& laruae \& imaginis omnino adhuc latent.

Obf. Genus Sphegibus affine, attamen charactere fatis diftinctum. 
rog. SPHEX. Linn. Ichneumon. Geoff. Os maxillis, palpis linguaque inflexa, trifida. Palpi quatuor, porrecti, fubaequales, fili. formes.

anteriores paullo longiores, fexarticulati: articulis fubaequalibus, obconi. cis adhaerentes maxillae dorfo.

pofteriores quinquearticulati : articulis aequalibus, obconicis labii medio laterali inferti.

Mandibula cornea, craffa, arcuata, acuta, dentata.

Maxilla porrefta, cornea, compreffa, la. tere interiori membranacea, in medio inflexa, apice obtura, integra linguae laminam exteriorem conftituens.

labium porrectum, corneum, cylindricum, apice elongatum, membrana. ceum, inflexum, emarginaturn vtrir. que feta membranacea, valida, longitudine dimidii labii interiorem linguae laminam conftituens.

Antcnnae breues, conuolutae, fetaceae, I articulatae: articulis aequalibus, cylindricis; primo paullo cralfiori. Larua apoda, mollis, veficulofa, intra corpora Infectorum latitans.

Puppa quiefcens imagini fimillima, folis alarum rudimentis.

Victus laruae e fucco infectorum, imaginis e floribus inprimis vmbellatis. 


\section{คำเป็}

\section{I เ. TIPHIA.}

Os maxillis palpisque absque lingua.

Palpi quatuor, inaequales, filiformes, porrecti.

antryores paullo longiores, fex articulati: articulis aequalibus adhaerentes maxillae dorf́o.

poferiores quinquearticulati: articulis aequalibus labii medio inferti.

Mandióula cornea, arcuata, acuta, integra.

Maxilla breuis, membranacea, apice ro. tundata, integra.

Labium breue, corneum, cylindricum, obrufum, ante apicem dentibus duobus erectis, obtufis, vel potius tridentatum dente interiore maiore, obtufiòre.

Antennae arcuatae, fubpetiolatae: articulo primo longiori, reliquis vix diftinctis, breuilfimis, rotundatis.

Metamorphofis \& Victus \& laruae \& imag: nis omnino adhuc nobis latent. 


\section{S S O L I A.}

Os maxillis, palpis, linguaque inflexa trifida, breuilfima.

Palpi quatuor, aequales, breues, filiformes.

antrriores quadriarticulati: articulis aequalibus, cylindricis adhaerentes maxillae dorfo.

pofteriores triarticulati: articulis aequalibus, globofis labii medio laterali inferti.

Mandibula cornea, arcuata, acutifima, in. tus crenata.

Maxilla porrecta, cornea, compreffa, apice obtufiufcula, integerrima, lacinias linguae exteriores conftituens.

Labium porrectum longitudine maxillae, corneum, compreffum, apice membranaceum, cylindricum, integrum, linguae laciniam interiorem conftituens.

Antennac craffae, filiformes, fubpetiolatae: articulo primo longiori, reliquis vix diftinctis, cylindricis, breuibus.

Metamorphojus \& Victus omnino adhuc latent. 


\section{*ลำ}

\section{II2. CHR YSIS. Linn. Vefpa Geoff.}

Os maxillis palpisque absque lingua.

Pulpi quatuor, porrecti, inaequales, filiformes.

anteriores longiores, quinquearticulati: articulo vltimo tenuiori, filiformi adhaerentes maxillae dorfo.

poferiores quadriarticulati: articulis aequalibus labii meciio inferti.

Mandibula exferta, cornea, arcuata, integra.

Maxilla porrecta, elongata, cornea, linearis, vnidentata, apice membranacea, acuta.

Labium porrectum, linguaeforme, corneum, lineare, maxillae multo longius, apice membranaceum, acutum, emarginatum.

Antennae breues, filiformes: articulo primo longiori, reliquis breuibus aequalibus.

Metamorphofis \& Victus adhuc latent. 


\section{II3. THYNNVS.}

Os maxillis, palpis linguaque breuifima, inuoluta, haud exferta.

Palpi quatuor, filiformes, aequales.

anteriores quadriarticulati: articulis ae. qualibus adhaerentes maxillae dorfo. poferioses triąrticulati: articulis fubaequalibus adnati labii medio.

Mandibuta cornea, incurua, apice acuta, vnidentata.

Maxilla breuis, recta, cornea, concaua, apice rotundata, integra.

Labimm maxillis longius, vsque ad infertionem palporum cylindricum, corneum, apice inflexum, membranace. um, compreffum, trifidum: laciniis aequalibus; intermedia emarginata.

Antennac cylindricae: articulo primo breui, craifiori, reliquis aequalibus, obfoletis.

Metamorpiogis \& Victus \& laruas \& imaginis adhuc latent. 


\section{LEVCOSPIS.}

Os maxillis, palpisque absque lingua.

Palpi quatuor, breues, aequales, filiformes: articulo vltimo reliquis longiori, obconico.

antrriores quadriarticulati: articulis fubcylindricis, aequalibus adhaerentes maxillae dorfo.

pofteriores triarticulati labii medio late. rali inferti.

Mandibule cornea, crafra, breuis, apice tridentata.

Maxilla labio breuior, cornea, compreffa, integra.

Labium elongatum, cylindricum, corneum, apice membranaceum, emarginatum.

Antenrae breues, rectae, cylindricae, extrorfun craffiores: articulis breuibus, cylindricis; primo paullo maiore.

Metamorphofis \& Viefus \& lasuae \& imaginis omnino adhuc latent. 
I 5. BEMBEX. Vefpa Linn. Apis Linn.

$O s$ maxillis, palpis linguaque inflexa quinque fida, labio fuperiore porrecto, conico, corneo obrectum.

Palpi quatuor, breues, inaequales, filiformes.

anteriores longiores, fex articulati: arriculis fubaequalibus; vltimo fubu. lato adhaerentes maxillae flexurae.

pofteriores breuiores, triarticulati bafi laciniarum exteriarum labii inferti. Mandibula recta, cornea, concaua, apice fubdentata.

Maxilla porrecta, fubcylindrica, cornea, medio infiexa, compreffa, obtufa, laciniam exteriorem linguae conftituens. Labium porrectum, bafi corneum, gibbum, trifidum: lacinis exterioribus tenuioribus filiformibus; intermedia maiori, fornicata, laterales includente, lacinias linguae intermedias conftiruens.

Antenuae filiformes, inuolutae: articulo primo longiore, cylindrico, reliquis breuibus, fere obfoleris.

Larua apoda, mollis, cylindrica.

Puppa quiefcens, imagini fimillima, rudimentis tantum alarum.

Vicius \& laruae \& imaginis e nedtare florum.

$B$. Jugnata lacinia media labii smarginata. I 6. VE. 


\section{Ir6. VESPA. Linn. Geoff.}

Os maxillis palpisque absque lingua. Palpi quatuor, inaequales, filiformes.

anteriores porrecti, longiores, fexarticulati: articulis fubaequalibus, obconicis; vltimo fetaceo adhaerentes maxillae dorfo.

pofteriores breuiores, quinque articulati: articulis fubaequalibus, cylindricis labii medio inferti.

Mandibula breuis, recta, cornea, lata, conca. ua, apice oblique truncata, denticulata. Maxilla porrecta, cornea, cylindrica, ad infertionem palpi flexa, membranacea, compreffa, vnidentata, apice acuta, ciliata.

Labium compreffum, bafi corneum, gibbum, carinatum, apice concauum, membranaceum, late emarginatum, medio vtrinque feta tenuiffima, filiformi, acuta inftructum.

Antcnnac filiformes, incuruae: articulo primo longiffimo, cylindrico, fecundo vix breuiori, reliquis breuibus, fere obfoletis.

Larua apoda, mollis: antice truncata, obtufa, poltice attenuata intra cellulas hexangulares.

Puppa quiefcens, imagini fimillima, rudimentis tantum alarum.

Victus laruac e nectare florum, melle, imaguis e rapina A pium, Mufcarum. 117 . 
I 7. CRABRO. Vefpa Linn. Sphex Linn.

Os maxillis palpisque absque lingua.

Palpi quatuor, porrecti, inaequales, filiformes.

anteriores longiores, fexarticulati: articulis compreffo rhomboidalibus; vlit. mis duobus cylindricis adhaerentes maxillae dorfo.

pofteriores breues, gibbi, quinquearticulati: articulis cylindricis, aequalibus Iabii apici laterali inferti.

Mandibula cornea, arcuata, fornicata, dentata, apice truncata, fiffa.

Maxilla recta, porrecta labio paullo longior, cornea, compreffa, bifida: la. ciniis fubaequalibus, rotundatis.

Labium breue, corneum, obconicum, depreffum, marginibus parum eleuatis, apice rotundatum, integrum.

Antensac filiformes, incuruae: articulo primo longiffimo, cylindrico, reliquis breuiffimis fere obfoletis.

Larua apoda, mollis.

Puppa quiefcens, imagini fimillima, rudimentis tantum alarum.

Victus laruae e nectare florum, melle, ima. ginis e rapina A pium, Mufcarum. 
118. ANDRENA. Apis Linn. Geoff.

Os maxillis, palpis linguaque inflexa trifida. Palpi quatuor, inaequales, filiformes.

anteriores porresti, longiores, fexarti. culati: articulis cylindricis, aequalibus adhaerentes flexurae maxillac.

pofteriores breuifimi, biarticulati : artictslis aequalibus, cylindricis fub apice fetarum labii inferti.

Mandibula recta, cornea, fornicata, inermis. Maxilla cornea, apice membranacea, compreffa, inflexa, labio multo breuiore linguae lacinias exteriores conftituens,

Labium porrectum, cylindricum, bafi corneum, apice membranaceum, in flexum, vtrinque iuxta flexuram fetis duabus membranaceis, rectis, rigidis: exteriore longitudine dimidii labii, in. teriore breuiffima, compreffa, incurua.

Antennac breues, filiformes, fubpetiolatae: articulo primo paullo longiori, fecundo bafi attenuato, reliquis aequa. libus, breuibus.

Larua apoda, mollis: antice gibba, obtufa, poftice attenuata.

Puppa quiefcens, imagini fimillima. Vičus \& laruae \& imaginis e nectare flo. rum. 
119. APIS. Linn. Geoff.

Os maxillis, palpis linguaque inflexa quin. quefida.

Palpi quatuor, breuirlimi, inaequales, filiformes.

anteriores paullo longiores, fexarticula. ti: articulis aequalibus flexurae maxillae adhaerentes.

poffriores quinquearticulati : articulis aequalibus, apici laciniarum exteriorum labii inferti.

Mandibula porrecta, cornea, recta, obtufa, dentata.

Maxilla porrecta, cornea: bafi cylindrica cum labio connata, apice comprefia, acuta, integra, marginibus membranaceis, in medio inflexa, laciniam lin. guae exteriorem confticuens.

Labium porrectum, corneum, bafi cylindricum, apice trifidum: lacinis lateralibus dilatatis, membranaceis, fub apice palpigeris, intermedia longiori retractili, tereti pilofa, lacinias interiores linguae conttiruéns.

Antennae filiformes, breues: articulo pri- 
mo longifimo, incuruo, reliquis breuibus, aequalibus.

Larua apoda, mollis, gregaria intra cellulas hexangulares.

Puppa quiefcens imagini fimillima.

Victus \& laruae \& imaginis e nectare flo. rum, melle, fructibus dulcibus.

Obf. Ap. longicornis \& linguaria labio gaudent quinquefido laciniis exterioribus dilatatis palpigeris, fecundariis minoribus tenuiffimis, interiori tereti pilofo. 
120. NOMADA. Apis Linr.

Os maxillis, palpis linguaque inflexa, trifidas Palpi quatuor inaequales, porrecti, filis formes.

anteriores breuiores, fexarticulati : articu. lis aequalibus, cylindricis adhaeren. tes flexurae maxillae.

poferiores quadriarticulati: articulis cy. lindricis fecundo longiflimo, lin. guae formi labii medio inferti.

Mandibula cornea, fornicata, arcuata, acuta, integra.

Maxilla bafi cornea, cylindrica, apice porrecta, compreffa, membranacea, acuta, integra linguae exteriorem laciniam conftituens.

Labium cylindricum, elongatum, bafi corneum, apice membranaceum, acutum, comprefium, integrum, linguac laciniam interiorem conftituens.

Antennae fliformes, breues, articulo primo paullo longiori, reliquis aequalibus, breuibus, rocundatis.

Mctamorphofis \& Victus \& laruae \& ima. ginis latent.

Obf. Genus affine Apibus; differt tamen pal. pis pofterioribus linguae formibus, quarum figura cylindrica \& articuli veros elle palpos indicant.

I2I. TER. 


\section{I2I. TERMES. Linn.}

Os maxillis palpisque absque lingua.

Palpi quatuor, aequales, filiformes.

anteriores vix longiores, quadriarticula-

ti: articulo primo breuiflimo, reli-

quis aequalibus, cylindricis maxillae medio larerali adhaerentes.

pofteriores triarticulati : articulo primo breuiori, reliquis aequalibus, cylindricis labii medio inferti.

Mandibula breuis, cornea, crafla, acuta, dentara.

Maxilia cornea, compreffa, incurua, bafi fquama maxilla breuiore, truncata, integra fuffulta, apice emarginata: denti. bus duobus validis, acutiffimis; poferiore longiore.

Zabium porrestum, corneum, bafi cylindricum, canaliculatum apice quadrifi. dum, laciniis linearibus, acutis.

Antennac moniliformes: articulo primo breui, craffiori.

Larua apoda, mollis, gregaria, lucifuga intra tubum cylindricum.

Puppa quiefcens, immobilis.

Victus laruae \& imaginis e rebus cibariis, culinariis, dometticis fere omnibus.

06). Neutra mandibulas gerunt exfertas, forcipatas, tenuiores. 
122. FORMICA. Linn. Geoff.

Os maxillis palpisque absque lingua. Pulpi quatuor, porrecti, inaequales, filiformes.

anteriores longiores, fex articulati: articulis fubaequalibus, cylindricis ad. haerentes maxillae medio.

poferiores quinquearticulati : articulis aequalibus, breuiffmis labii apici inferti.

Mandibula recta, cornea, lata, fornicata, apice obtuia, denticulata.

Maxilla fubmembranacea, arcuata, fornicara, comprefra, obtufa, integra.

Labium porrectum longitudine maxillae, fubmembranaceum, cylindricum, truncatum, integrum.

Antennac filiformes: articulo primo longiori, cylindrico, reliquis breuibus, vitimo feraceo.

Lama apoda, mollis, alba, antice po. ficeque obtufa.

Pripa imnobilis, quiefcens.

Vicfus \& laruae \& imaginis e quisquiliis, rapina, faccharo, fructibus aliisque.

Maribus minoribus alatis, faeminis maioribus alatis \& fpadonibus apteris conftat Formicarun refpublica. 


\section{I23. MVTILLA. Linn.}

Os-maxillis palpisque absque lingua.

Palpi quatuor, porrecti, inaequales, filiformes.

antcriores longiores, fex articulati: articulo tertio longiffimo, reliquis aequalibus, obconicis: angulo interiori prominulo, vltimo fetaceo maxillae apici adhaerentes.

pofteriores breuiores, quinquearticulati : articulo fecundo longilimo, obco. nico, quarto breuiffimo, latiori labii apici inferti.

Mandibula cornea, fubarcuata, acuta, integra.

Maxilla breuis, fubcylindrica, recta, cor- nea, apice membranacea, integra,obtufa.

Labium corneum, obconicum, gibbum, truncarum, integrum, longitudine maxillae.

Antennac filiformes: articulo primo longiori, incuruo, reliquis breuibus aequalibus.

Metanorphofis \& Vißtus \& laruae \& imaginis omnino adhuc latent. 


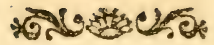

Clafis IIII.

\section{A GONA TA.}

$O$ s mandibulis palpisque absque maxillis.

Palpi fex inaequales, filiformes: anterioribus quatuor longioribus bafi bificis, apice incuruis os obtegentibus, pofticis mandibulae dorfo infertis.

Clypeus breuis, corneus, rotundatus, vix fupra os exfertus.

Mandibulae duae transuerfae, corneae, craffae, dorfo palpigerae.

Maxillae omnino nullae.

Labium triplex, membranaceum, rotun: datum, diuifum.

Antennae quatuor, fub oculis infertae. 


\section{พจำ}

GENERA Claffis IIII. 12z. Seorpio. videroff.136.

125. CANCER.

I26. PAGVRVS.

127. SCYLLARVS.

128. ASTACVS.

I29. GAMMARVS.

$$
\text { I } 3 \text { I25. CAN }
$$


125. CANCER. Linn.

Os mandibulis palpisque absque maxillis.

Palpi fex inaequales: anterioribus quatuor, compreffis latis totum os obte. gentibus.

anteriores bifidi : laciniis longitudine aequalibus; interiori quaciriarticulata: articulo primo longiori, fecundo fuborbiculato, vltimo acuminato, exteriori biarticulata: articulis aequalibus.

medii bifidi: laciniis inaequalibus; interiori latiori, breuiori, triarticulata: articulo fecundo longiori, exteriori longiori, tenuiori, biarticu. lato: articulis aequalibus; vltimo incuruo.

Pofteriores breues, incurui, triarticulati: articulis aequalibus adhaerentes mandibulae dorfo.

Mandibula breuis, craffa, cornea, apice rotundata, fornicata, obtufa.

Iabium triplex: exterius octofidum, laciniis interioribus breuilfimis, cylindricis, fecundis tertiisque aequalibus, apice elongatis, fubulatis, quartis latiori- 


\section{*ลำ}

bus truncatis, apice exteriori feta fubulata, longifima, tenui, acutiffima.

medium quadrifidum, laciniis intermediis latiffinis, breuioribus truncatis, filfis; exterioribus tenuioribus dorfo retigeris apice fubulatis porrectis.

interius paruum, quadrifidum : laciniis aequalibus; interioribus extrorfum craffioribus forncatis, truncatis, exterioribus cylindricis.

Antenuse quatuor breuifimae, aequales.

exteriores latae, petiolo lacui, vnidentaro.

intermedii palpiformes, quadriarticulati, articulo fecundo longiori, vltimo acuminato, bifido.

Metamorpbofis completa larua puppaque omnibus partibus completis agilibus.

Vitus e rapina infectorum, ranarum $\mathrm{pi}$. fciumque. 
126. PAGVRVS. Cancer Linn.

Os mandibulis palpisque absque maxillis.

Palpi fex fubaequales: anterioribus quatuor totum os obtegentibus.

anteriores porrecti multo longiores, bifidi: laciniis inaequalibus; exteriori breuiori, quadriarticulata: articulo fecundo longioni comprefio, vitimo acuto annulato, interiori longioni, fexarticulara: articulis fubaequalibus, pilofis; fecundo extrorfum ferrato fub ore inferti.

medii breuiores, bifidi: laciniis aequa. libus; exteriori acutifima, quadriarticulata : articulo fecundo longisfimo, interiori guinquearticulata: articulis compreffis, ciliatis.

pofteriares parui, triarticulati: articulo vltimo craffiori, compreffo nandibulae dorfo inferti.

Mandibula cornea, craffa, valida apice ro. tundata, obtufa, fornicata.

\section{Labium triplex.}

exterius quadrifidum: laciniis interio. ribus rotundatis, fornicatis, intus ciliatis, exterioribus rectis, fubconicis, 


\section{भत⿵}

nicis, apice bafique interiori feta fubulata, acuta palpiformi infructis.

medium quadrifidum : laciniis fubaequalibus, fornicatis, interioribus bafi interiori inclis, ciliatis; exterioribus apice fubulatis bafi interiori feta rigida acutifima infructis.

intcrius quadrifidum: laciniis interioribus rotundatis, arcte mandibula adhaerentibus, exterioribus longioribus, extrorfum crallioribus, ciliatis dorfo feta valida infructis.

Antenme quatuor, inaequales, pedunculatae. cxteriores longiores, fubularae, multiarticulatae: pedunculo craffiori triarticulato; articulo fecundo fpinofo.

intcriores brenes, palpiformes, triarti. culati : articulo fecundo longiori, vlrimo bifido, pedunculo vniarticu. lato, gibbo, fpinolo.

Metamorpbofis completa, larua puppaque onnibus partibus completis, agilibus.

Vidus c rapina infectorum, ranarum pifciumque. 
127. SCYLLARVS. Cancer. Linn.

Os mandibulis palpisque absque maxillis.

Palpi fex inaequales; anterioribus quatuor, porrectis obtegentibus.

anteriores longiores, quadriarticulati: articulis compreffis, inaequalibus; primo tertioque breuioribus, bafi exteriore fquamula breui cornea, arcuata, inarticulata, acuta armatis.

medii bifidi : laciniis fubaequalibus; interiore triarticulara: arriculis compreffis, breuibus, vltimo incuruo, exteriore biarticulata, erecta, obtufa.

Poferiores breues, fetacei, triarticulati, incurui mandibulae dorfo inferti.

Mandibula porrekta, cornea, intus canaliculata bafi fubcylindrica apice incralla. ta, concaua fubemarginata.

Labium triplex.

exterius quadrifidum: laciniis rotundatis, fubaequalibus.

medium bifidum: laciniis fiffis, aequalibus. 
interius bifidum: laciniis extrorfum craffioribus, trifidis.

Antennac quatuor, inaequales.

anteriores paullo longiores, filiformes, quadriarticulati: articulis fubaequa. libus; vltimo breuiori bifido.

poferiores breues, bifidi : lacinia interiori biarticulata; articulo primo rotundato breui, vltimo magno, compreffo, rotundato, crenato, la. cinia exteriori inarticulata, com. prefra, carinata, acuta margineque fpinofo.

Metamorplofis completa, larua puppaque omnibus partibus completis, agilibus.

Victus e rapina infectorum aquatilium pi. fciumque minimorum. 
140

I28. ASTACVS. Cancer Linn. Geoff.

Os mandibulis palpisque absque maxillis.

Palp: fex, inaequales: anterioribus quaiuor porredtis, os obtegentibus.

anterines longiores, bifidi: laciniis inaequalibus; interiore longiore, qua. driarticulata: articulo fecundo tertioque longioribus, exteriori breui, acutillima, triarticulata.

medii bifdi: laciniis aequalibus; interiore, craffiori, triarticulata: articulo fecundo breuifimo, vltimo cratiori, inflexo, extcriori fubula. ta, tenuiori, inarticulata.

pofteriores breues, fetacei, triarticulati mandibulae dorfo inferti.

Mandiouda breuis, crafra, cornea, bifida: facina interiore cylinarica, refta, apice dentata, exteriore fornicata, apice truncata, dentata.

Labium triplex.

sxterius quadrifidum: iaciniis fubaequalibus, interioribus inferne ferratis, exterioribus fiffis, apice feta fubula. ta, porrecta, acuta. 
medium bifidum: laciniis rotundatis, tri. fidis; pinna media acutiore, bre. uiore.

interius bifidum : laciniis extrorfum craffioribus, quinquefidis; pinna media longiore.

Antennae quatuor, inaequales, multiarticulatae, pedunculatae.

anteriores longiores, fetaceae, muitiarticulatae: pedunculo articulato, craf: fo, fpinofo.

poferiores multiarticulatae, ad bafin fiffae: pedunculo articulato, fpinofo. Mctamorpbofis completa, larua puppaque omnibus partibus completis, agilibus.

Victus e rapina infectorum aquatilium, d: narum pifciumque. 
129. GA MMARVS. Cancer. Linn. Geoff. Onifcus Pallas.

Os mandibulis palpisque absque maxillis.

Palpi fex, inaequales, filiformes: ante. rioribus quatuor porrectis, os obtegentibus.

anteriores longiores, compreffi, bifidi: lacinia interiori quadriarticulata ; 21 ticulo vltimo incuruo, exteriori breuiori, vix articulata.

medii paullo breuiores, bifidi : laciniis fubaequalibus; interior triarticula. ta, exteriori fubulata, acuta.

pofteriores breues, filiformes, triarticu. lati mandibulae dorfo inferti

Mandibula breuis, cornea, craffa, fornicata, obtufa, vix dentata, dorfo palpigera.

Labium triplex membranaceum.

exterius quadrifidum: laciniis fubae. qualibus, linearibus.

medium bifidum : laciniis rotundatis, diuifis, aequalibus. 
interius bifidum: laciniis aequalibus, rotundatis, extrorfum craffioribus, diuifis.

Antennac quatuor inaequales, peduncula. tae, fimpliciffimae.

anteriores breuiores, fubulatae: pedunculo biarticulato.

pofteriores longiores, fetaceae: pedunculo triarticulato.

Metamorpbofis completa larua puppaque omnibus partibus completis, agilibus.

Victus e rapina minimorum aquatilium plantisque aquaticis. 


\section{Clajis $V$. \\ VNOGATA.}

Os maxillis palpisque.

Palpi duo, breues, faepius filiformes, interdum maxillae inferti, incumbentes, interdum palato inter mandibulas maxillasque adhaerentes.

Clypeus horizontalis, corneus, rotundatus, os fuperne tegens.

Mandibulae duae transuerfales, cornea, mobiles, breues, gibbae, interdum concauae, vnguiculatae latera oris $\mathrm{fu}$. perne includentes.

Maxillae duae,transuer fales, mobiles, membranaceae, faepius vnguiculatae, latera oris inferne includentes.

Labium porrectum, liberum, os inferne claudens.

Antennae breuiftimae, filiformes fub oclilis infertae. 


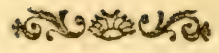

\section{GENERA Claffis V.}

130. LIBELLVLA.

131. AESHNA.

132. AGRION.

133. IVLVS.

134. SCOLOPENDRA.

35. TROMBIDIVM.

36. ARANEA.

124. SCORPIO.

37. PHALANGIVM. 
130. LIBEL L VLA. Linn. Geoff.

Os maxillis palpisque.

Palpi duo fliformes, multiarticulati: articulis obfoletis, vix diftinguendis ma. xillae dorfo inferti, cui fuperincumbunt.

Mandibula breuis, cornea, crafra, gibba, bafi dentata, apice acuminata.

Maxilla porrecta, cornea, compreffa, bafi dilatata, ciliata, apice vnguiculata, acutifima, dentata.

Labirm porreçum, exfertum, membranaceum, trifidum: laciniis lateralibus maioribus, fornicatis, rotundatis, ciliatis totum os obtegentibus, tertia dorfali, minutiffima, rotundata, vix emarginata.

Antennac breues, filiformes: articulis vix dittinguendis, breuiffimis.

Larua hexapoda, agilis: ore forcipe articulato, bifido, membranaceo tecto.

Puppa hexapoda, agilis, laruae fimillima at rudimentis alarum.

Victus \& laruae \& puppae e rapina infectorum aquatilium, imaginis e rapina Antliatorum. 
I3r. AESHNA. Libellula Linn. Geoff.

Os maxillis palpisque duobus.

Palpi dro, porrecti, pilofi, fubcornei, incumbentes: articulis plurimis, breuiffomis, obfoletis longitudine maxillae, maxillae dorfo inferti.

Mandibula craffa, cornea, arcuata, denta. ta, exferta.

Maxilla cornea, valida, compreffa, arcuata, dentata, apice bifida: dente interiori mobili.

Labium porrectum, fornicatum, fubmem. branaceum, trifidum: lacinia intermedia rotundata, canaliculata apice emarginata; lateralibus ouatis, oblique trun. catis, dentatis: dente fuperiori maiori, validiori, inflexo.

Antennae breues, filiformes: articulis ob. foletis vix diftinguendis, breuiffimis.

Lavua hexapoda, agilis, currens: ore forcipe articulato, vnguiculato, acuto, tecto.

puppa hexapoda, agilis laruae fimillima at alarum rudimentis.

ficfus \& laruae \& puppae e rapina infeEtorum aquatilium, imaginis e Antlia. torum.

$$
\text { K } 13 .
$$


I32. A GRION. Libellula Linn. Geoff.

Os maxillis palpisque.

Palpi duo breues, filiformes, multiarticu. lati : articulis fubobfoletis, vix diftin. guendis maxillae dorfo inferti, cui fu. perincumbunt.

Mandibula breuis, crafa, cornea, gibba, apice acuminata, dentata.

Maxilla porreita, cornea, comprefla, bafi dilatata, apice vnguiculàta, acu. tiffima, dentata.

Labium porredum, membranaceum, ro. tundatum, trifidum: laciniis exterio. ribus tenuiffmis, fublongioribus, apice bifidis, acutifimis, intermedia maiori, latiore, apice emarginata.

Antenna brenes, filiformes: articulis obfoletis, vix diftinguendis, breuiflimis.

Larua hexapoda, agilis, currens: ore forcipe articulato, bifido; lacinia exterio. ri vnguiculis pluribus armato, te£to.

Puppa hexapoda, currens, laruae fimillima at alarum rudimentis.

Victus \& lartiae \& puppae e rapina infecto. rum aquarilium, imaginise Antliatorum. 
I33. IVLVS. Linn. Geoff.

Os maxillis palpisque.

Palpi duo, breues, filiformes, comprer.

fi, connati, quadriarticulati: articulo fecundo longiori, vltimo ouato obtufo, inter mandibulas maxillasque inferti.

Mandibula breuis, craffa, gibba margine interiori ferrata apice obtufa, bifida.

Maxilla breuis, membranacea, filiformis, integra.

Zabium magnum, porrečum, membranaceum, rotundatum, apice crenatum, emarginatum.

Antennac filiformes, fexarticulatae: articulis aequalibus, fubmoniliformibus, vltimo obtufo.

Metamorpiofis completa.

Larua polypoda, agilis, currens matri fimillima.

Puppa polypoda, agilis, imagini fimillima.

Victus \& laruae, \& puppae \& imaginis e rapina infectorum minimorum, Aca. rorum, aliorumque. 


\section{SCOLOPENDRA. Linn. Geoff.}

Os maxillis palpisque.

Palpi duo aequales, connati, comprefli, quadriarticulati: arriculo fecundo longifimo, vltimo acutiffimo, fubulato palato inter mandibulas maxillasque inferti.

Mandibula breuis, fornicata, bafi anuulata, apice obtufa, rotundata, fifia.

Maxilla magna, porre£ta, cornen, arcuata, acutiffima, bafi vnidentata.

Labinin breue, corneum, rigidum, apice truncatum, dentatum, bifidum: laciniis aequalibus, obtufis.

Antenna porrectae, fetacese, multiarticulatae: articulis aequalibus, breuibus; fecundo paullo maiore.

Metamorphofis completa.

Larua polypoda, agilis, currens, ima. gini fimillima.

Puppa polypoda, agilis, currens imagini fimillima.

Vis̃us omnium e rapina infectorum minimorum. 
135. TROMBIDIVM. Acarus Linn.

Geoff.

Os maxillis palpisque.

Palpi duo porrecti, incurui, maxillas aemulantes, filiformes quadriarticulati: articulis cylindricis; fecundo gibbo, craffiori, vlrimo corneo, acutiffimo, arcuato os obtegentes.

Mandibula recta, porrecta, compreffa, bafi excauata, apice vnguiculo corneo, arcuato, acutifimo armata.

Maxilla breuifima, incurua, approximata.

Labium porrectum, conicum, membrana. ceum, concauum, inuolutum, pilo. fum, maxillas recipiens.

Antennae, quantum video, omnino nullae. Metamorphofis completa.

Larua octopoda agrilis, currens, omnibus partibus completa, imagini fimillima.

Puppa oćtopoda, agilis, currens imagini fimillima.

Victus \& laruae \& puppac \& imaginis rapina aliorum infectorum, quisquiliis. 


\subsection{AR A NEA. Linn. Geoff.}

Os maxillis palpisque.

Palpi duo porrecti, aequales, incurui, qua. driarticulari : articulo fecundo longiffi. mo, vltimo obtufo maxillae dorfo ad. haerentes.

Mandibula breuis, craffa, cornea, appro. ximara, caua, cylindrica, apice vnguiculata, acutifima.

Maxilla breuis, cralla, cornea, cylindrica, apice vnguiculo arcuato, acutifimo armata.

Labium breue, membranaceum, apice rotundatum, integrum.

Antennac, quancum videre licet, omnino nullae.

Mctamorpionis completa.

Larua octopoda, agilis, currens, omnibus partibus completa, imagini fimillima.

Puppa octopoda, agilis, currens imagini fimillima.

Dicius \& laruae \& puppae \& imaginis e rapina aliorum infectorum inprimis Antliatorum.

Ob. I. Palpi maris articulo vltimo clauato, veficulofo apice acuminato funt ge. nitalia.

06). II. Situs oculorum in fpeciebus differt $\&$ fubdiuifiones generis diftineturimas praebet. 


\section{.6.}

124. SCORPIO. Linn. Chelifer. Geoff.

Os maxillis palpisque.

Palpi duo elongati, cheliferi, quadriarticulari: articulis cylindricis; primo breuiffimo, vltimo ad medium bifido: la. ciniis conniuentibus, aequalibus, exteriori articulato, mobili fub maxilis inferti.

Mandibula breuis, craffa, cylindrica, dorfo gibbo, apice vnguiculata: vnguibus duobus arcuatis, acutifimis.

Maxilla breuis, cornea, fornicata, apice vnguiculo arcuato, acuto labium includens.

Zabium rectum, porrectum, corneum, bi. fidum: laciniis aequalibus, obtufis. Antennae, quantum video, omnino nullae. Metamorphofis completa.

Larua octopoda, agilis, currens omnibus partibus completa, imagini fimillima.

Puppa octopoda, agilis, currens, imagi. ni fimillima.

Victus \& laruae \& puppae \& imaginis rapina infectorum ver miumque. 


\section{PHALANGIVM. Linn. Geoff.}

Os maxillis palpisque.

Pulpi duo aequales, incurui, elongati, fcabri, filiformes, quadriarticulati: articulo fecundo quartoque longioribus, tertio interne bidentato maxillae dorfo inferti.

Mandibula porrecta, cornea, arcuata, rigida, biarticulata: articulo fecundo dente mobili chelifero acutiffimo.

Maxilla breuis, cylindrica, membranacea, caua, apice truncata, obrufa.

Labium breue, rotundarum, membranaceum \& fere veficulofum, bificim: laciniis rotundatis, aequalibus.

Antennae, quantum videre licet, omnino nullae.

Metamorphofis completa.

Larua odtopoda, omnibus partibus com. plera imagini fimillima.

Puppa octopoda, agilis, currens, imagini fimillima.

$V i c t u s$ \& laruae \& puppae \& imaginis e rapina aliorum Infectorum, vermiumque.

065. Pbalangii cornuti articulus palporum penultimus digito mobili palpiformi in. fructus. 


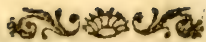 \\ Clajis VI. \\ G L O S S A T A. Züngler}

$O$ s lingua fpirali palpisque absque maxillis, mandibulis labioque.

Clypgus breuiffimus, corneus, rugofus, integer, vix bafin linguae f piralis tegens. untisution.

Palpi duo, interdum quatuor, compreffi, outat, pilofi, reflexi os obtegentes fub ore inferti.

Lingua fpiralis porrecta, inuoluta, fetacea, mox cornea, mox membrana. cea, bifida : laciniis aequalibus, acutis, intus canaliculatis inter palpos inferta, latens.

Antennae elongatae.

06f. Characteres generum huins claffis mihi nondum fatis fecerunt. 
GENERA Claflis VI. sobomis.

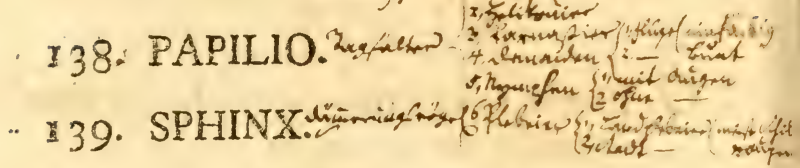
140. SESIA. fminnom?

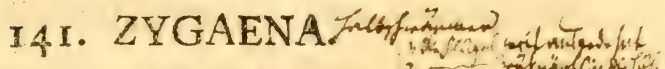

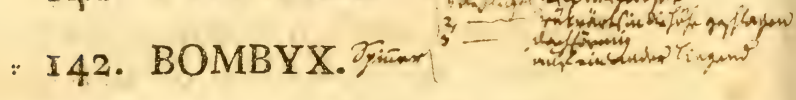

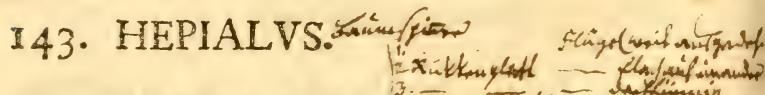

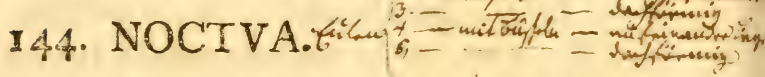

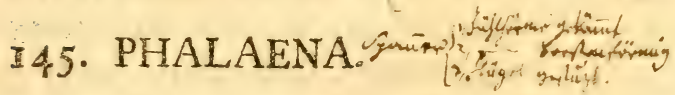
i46. PYRALIS. jumation 147. IINEA. morthw 443. ALVCITA gemsing pelau I49. PTEROPHORVS. vandariytw. 
138. PAPILIO. Linn. Geoff.

os lingua fpirali palpisque absque maxillis.

Palpi duo aequales, comprefi, ouati, acuti, pilofi, reflexi os obregentes fub ore inferti.

Lingua Piralis porreita, clongata, cor. nea, inuoluta, fetacea, bifida: laciniis aequalibus, acutis, intus canalicu. latis inter palpos inferta, latens.

Clypeus breuis, corneus, conicus, acu. tus, integer, rugofus, vix bafin lin. guae exteriorem tegens.

Antennae elongatae multiarticulatae, cla. uatae: claua capitata, ouata, obrufa.

Larua XVI poda, agilis, currens, fpinis compofitis faepius armata, interd:ams nuda, tentaculata.

Puppa nuda, quiefcens, plerumque nodofa interdum filis quibusdam apicis, interdum filis in medio alligatis ad. haerens.

Victus laruae e foliis plantarum, imaginis e nectare florum fuccoque arborum exfudante.

Obf. Equitum plures fpecies antennas gerunt filiformes absque omni claua.

139. SPHINX 
139. SPHINX. Linn. Geoff.

Os palpis linguaque (pirali absque maxillis.

Palpi duo reflexi, compreffi, aequales, ouati, obtufi, hirfutiffimi fub ore inferti os tegentes.

Lingua fpiralis, cornea, porrecta, exferta, inuoluta, filiformis, truncata, ob. rufa, bifida: laciniis intus canaliculatis fupra palporum bafin inferta.

Clypeus breuis, corneus, truncatus, fornicatus, integer linguae bafin fuperne tegens.

Antennae filiformes multiarticulatae : articulis aequalibus, fubrus fquamula parua, erecta, obtufa armatis.

Larua XVI poda, agilis poftice plerum. que cornu erecto rigido, acuto armata. Puppa quiefcens, nuda, lacuis, glabra, poftice obrufiffima.

Vičus laruae e foliis plantarum, imaginis e nectare florum.

Obf. S. ocellatae lingua breuiffima, haud exferta. 
140. SESIA. Sphinx Linn. Geoff.

Os palpis linguaque fpirali absque maxillis.

Palpi duo, compreffi, aequales, ouati, acuminati, pilofi fub ore inferti torum os tegentes.

Lingua Jpiralis cornea, exferta, porrecta, inuoluta, filiformis, obtufa, truncata, bifida: laciniis aequalibus, obtufis, intus canaliculatis fupra palporum bafin inferti.

Clypcus breuis, corneus, rotundatus, apice acuminatus, vix bafin linguae tegens.

Antennae cylindricae, extrorfum craffio. res, multiarticulatae: articulis aequa. libus.

Larua XVI poda, agilis, plerumque nuda, inermis, antice attenuata.

puppa quiefcens, nuda, laeuis, antice pofticeque acuminata.

Viftus laruae e foliis plantarum, imaginis e nectare florum. 
141. ZY GAENA. Sphinx I.inn. Geoff.

Os palpis linguaque fpirali absque maxillis.

Balpi duo aequales, compreffi, reflexi, pilofi, ouati, acuminati fub ore inferti totum os tegentes.

Iingua fpiralis cornea, exferta, porrecta, inuoluta, fetacea, acuta, bifida: laciniis aequalibus, fetaceis, acutis, intus canaliculatis fupre palporum bafin inferti.

Clypeus breuis, corneus, rotundatus, in. teger vix linguae bafin tegens.

Antennac porrectae, multo articulatae, ante apicem faepius craffiores, apice acuminatae.

Larua XVI poda, agilis, craffa, obefa, pilis breuibus tecta.

Puppa quiefcens, folliculata, antice pa. rum attenuata.

Victus laruae e foliis plantarum, imaginis e nectare florum.

Obf. Species quaedam antennas gerunt filiformes, pectinatas.

742. BOM: 


\section{+ลำ}

I42. BOMBYX. Phalaena Linn. Geoff.

Os palpis linguaque fpirali absque maxillis.

Palpi duo aequales, compreffi, pilofi, reIlcxi, obtufi, fub ore inferti, totum os obregentes.

Lingua fpiralis breuis, membranacea, vix exferta, filiformis, obtufa, bifida: $]_{2}$ ciniis aequalibus, filiformibus, fubcy. lindricis, intus canaliculatis fupra pal. porum bafin inferti.

Clypeus breuis, corneus, hirtus, apice acuminatus, linguae bafin fuperiorem regens.

Antennae filiformes, multiarticulatae, apice acutae.

Larua XVI poda, agilis, currens, faepius pilofa, fubcylindrica.

Puppa quiefcens, folliculata, cylindrica, apice acuminata.

Victus laruae e foliis plantarum, imaginis e nectare florum.

Ob\%. Antennae femper filiformes, maris faepius pectinatae. 
r.3. HEPIALVS. Phalaena Linn. Geoff.

Os palpis, linguaque fpirali absque maxillis.

Palpi duo aeguales, reflexi, obufi, menn. branacei, comprefii, hirfuti, fub ore inferti, os obtegentes.

Lingua fpiralis rudimentum brene, membranaceum, latum, cieprefrum, bifidum: laciniis aequalibus, obtufis, rotundatis inter palpos infertum.

Clypous breuis, corneus, rotundatus, integुer, linguae bafm fuperne tegens.

Antennse breues, filiformes: articulis plt:rimis, diftinctis, aegualibus, rotundatis.

Larua XVI poda, agilis, currens, fubcy. lindrica, faepius glabra.

Puppa quiefcens, folliculata, cylindrica, apice acuminata.

Victus laruae e radicibus plantarum, imagi. nis e nectare florum. 


\section{เง}

Iax. NOCTVA. Phalaena. Linn. Geoff. Os palpis linguaque fpirali absque marillis. Polpi cino, acquales, refexi, comprefi, pulofi, apice cylincrici, nudi, outuli, menbranacei, fub ore inferti, os obte. gentes.

Ingra pirilis cornea, exferta, porredta, inuoluta, fetacea, acuta, bifuda: laciniis aequalibus fetaceis, acutis, intus canaliculatis inter palpos inferta.

Ciprear brenis, corneus, rotundarus, in. reger, vix linguae fpiralis bafin fuperio. rem tegens.

Antcrane fetaccae, articulis pitimis aequalibus, cylindricis, vix difinguendis, vltimo acuto.

farua XVI- poda, agilis, currens, fub. cylindrica, faepius glabra.

Puppa folliculata, cylindrica, apice acu. minata.

Fitus laruae e foliis plantarum, imaginis - nectareforum.

Obf. Anternae plerumque fimplices, maris rarius pectinarae.

$$
\text { I. } 345 . \mathrm{PHA} \text {. }
$$


164 मतै

145. PHAL A ENA. Linn. Geoff.

Os palpis linguaque fpirali absque maxillis.

Palpi duo aequales, reflexi, compreffi, membranacei, ftupofi, fisbnudi, cylindrici, obtufi, fub ore inlerti os te. gentes.

Lingua (piralis membranacea, exferta, fe. tacea, inuoluta, bificla: laciniis aequalibus, fetaceis, intus canaliculatis fupra bafin palporum inferta.

Clypeus breuis, corneus, rotundatus, integer bafin linguae fuperne tegens.

Antennae filiformes: articulis plurimis, breuibus, aequalibus, obfoletis.

Larua VIII - X poda agilis, currens, cylindrica, glabra.

Puppa folliculata, cylindrica, apice acuminata.

Victus laruae e foliis plantarum, imaginis e nectare florum.

Obf. Subdinifio fpecierum in pectinicornes $\&$ feticornes vix valet, guum mas faeminae feticornis faepius pectinicornis. 
146. PYR ALIS. Phalaena. Linn. Geoff.

Os palpis linguaque fpirali absque maxillis.

palpi duo aequales, nudi, ftupofi, reflexi, bafi cylindrici, medio ouati, dilatati, apice fetacei fub ore inferti tocum os obtegentes.

Lingua piralis porreeta, membranacea, inuoluta, fetacea, bifida: laciniis aequa. libus, fetaceis, acutis fupra palporum bafin inferta.

Clypeus brevis, corneus, truncatus, latus, integer vix balin linguae fuperne tegens,

Antennac filiformes, fimplices: articulis breuibus, aequalibus, obfoletis.

Larua XVI poda, agilis, currens, plerum. que intra cucullum e foliis plantarum contortis filis paucioribus connexis latens.

Puppa quiefcens, cylindrica, antice acuminata, poftice obtufa.

Vicfus laruae e foliis plantarum; imaginis e nectare florum.

$$
\text { L } 3 \quad 147 . \mathrm{TI}
$$


147. TINEA. Geoff. Phalaena Iind

Os palpis linguaque fpirali absque maxillis.

Patpi quatior, inaequales.

shteriores tongiores, poriesti, filifor mes, cylindrici, membranacei, obtufi, bdi nudi, fubarticulati, apice fubhir futi,fubore infertiostegentes.

pofterieres multo breviores, nudi, cylindrici, obtufi, bifidi: lacinis aequalibus vtrinque ad bafin linguae inferti.

Lingua fpiralis membranacea, exferta, fetaces, bifida: laciniis acqualibus, fe. taceis, intus canaliculatis inter palpos inferiores inferta.

Clypeus breuis, corneus, rotundatus, in. teger, pilofus linguae bafin tegens.

Artenza fetaceae : articulis plurimis, ae qualibus, breuifimis, obfoletis.

Laria XVIs poda, agilis, currens, intra cat cullum faepius latens.

Puppa quiefcens, fubcylindrica, antice acuminata.

Victus laruac e folits piantarum, rebus cu. linariis, veftimentis, pellibus alisque. 


\section{भลำ}

148. A V CITA. Phalaena Linn. Tinea. Geoff.

Os palpis linguaque fpirali absque maxillis.

Balpi duo reflexi, porreeti, cylindrici, nudi, acquales, membraracei, acuri, vsque ad medium bifidi: laciniis fub: acqualibus linearibus; interiori acutiffima, nuda, exteriovi hirfuta, fub ore inferti os tegentes.

Lingua fpiralis membranacea, porrecta, fetacea, inuoluta, bifida: laciniis aequa. libus, fetaceis, intus canaliculatis ad bafin palporum inferta.

Clypers porredus, corneus, rotunclatus, hirtus, integer bafin linguae tegens.

Antenne fetaceae: articulis breuibus, plurimis, aequalibus vix diftinguenciis.

Lavua XVI- poda agilis, currens, faepius intra cucullum larens.

Puppa quiefcens, fubcylindrica, antice acuminata.

Thifus laruae e foliis plantarum. 


\section{PTEROPHORVS. Geoff. Phalaena Linn.}

Os palpis linguaque fpirali absque maxillis.

Palpi duo tenuifirri, cylindrici, filifor. mes, reflexi, nudi, apice fubulati, acuti, membranacei fub ore inferti totum os tegentes.

Lingua fpiralis membranacea, porrecta, elongata, fetacea, acuta, bifida: laciniis aequalıbus, intus canaliculatis, acutis fupra palporum bafin inferta.

Clypeus porrectus, membranaceus, truncatus, nudus, obtufus, integer linguae bafin tegens.

Antennae fetaceae: articulis plurimis breuiffimis, aequalibus vix diftinguendis.

Larua XVI- poda, agilis, currens, ouata, pilofa.

Puppa quiefcens, nuda, fubcylindrica apice fubulata.

Victus laruae e foliis plantarum, imsginis vix vllus. 


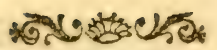

\section{Clafis VII.}

\section{R Y N GO T A.}

$O$ s roftro absque maxillis, mandibulis palpisque.

Roftrum inflexum, cylindricum: vagina vniualui, articulata, obtufa fetisque plerumque tribus, filiformibus, aequa. libus, acutis.

Labium porrectum, corneum, acutum, rigidum, roftri bafin fuperne tegens.

Antennae breues, faepius filiformes, tenuiffimae.

Pulex a reliquis generibus huius claffis dif. fert vagina biualui, fera vnica, laminis duabus ad vaginae bafin infertis \& me. tamorphofi. 
GENERA Clanis VIL

I50. FVLGORA.

15 1. MEMBRACIS.

153. TETTIGONIA.

53. CICADA.

154. CERCOPIS.

155. NOTONECTA

I56. SIGARA.

557. NEPA.

558. NAVCORIS。

59. ACANTHIA.

160. CIMEX.

I6I. REDVVIVS.

162. PVLEX.

163. APHIS.

164. CHERMES.

165. COCCVS.

¡66. THRIPS. 


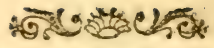

150. FVLGORA. Linn.

Of roftro absque maxiliis palpisque.

Roftrum elongatum, inflexum, filiforme; obtufum.

Datina vniualuis, cornea, cylindrica, fupra canaliculata, quinque articulata: articulorecundo breuiflimo, quarto longifimo, vltimo breuiori obtufo.

Setre tres intra vaginam retractiles, filiformes, acutae; intermedia fub. longiori.

Lavium porrectum, corneum, rigidum acutum, carinatum, fuperne roftrum tegens.

Antemae breues, craffe, in fouea capitis recondendae, capitatae.

sipes aniulatis, retractilis, bafin $\mathrm{ca}$. pituli vaginans.

Capitulun oblongum, apice excauato retufum, feta filiformi acuta longitudine antennarum.

Laruc hexapoda, agilis, currens, imaginifimillima at aptera.

Pup ha hexapoda agilis, currens imagini fimillima at folis alarum rudimentis.

titus e fucco plantarum, quem roftro haulit. 
15 r. MEMBRACIS. Cicada Linn.

Os roftro absque maxillis palpisque.

Rofrum inflexum, obtufum.

Vagina porrecta, vniualuis, cylindrica, obtufa, fuperne canaliculata, triarticulata: articulo primo brevifimo, reliquis aequalibus fub fetis inferta.

Setae tres aequales, conniuentes, arete fibi inuicem adhaerentes, fubulatae, in vaginae canali recondendae ad bafin vaginae infertae.

Labium elongatum, corneum, conicum, rigidum apice membranaceum, fubulatum, acutum, fetarum baifin tegens.

Antennae tenuiffimae, breues, fubulatae fronti infertae.

Larua hexapoda, currens, imagini fimil. lima, aptera.

Puppa hexapoda, currens, imagini fimilli$\mathrm{ma}$ at rudimentis tantum alarum.

ViCZus \& laruae \& puppae \& imaginis e Iucco plantarum. 


\section{I52. TETTIGONIA. Cicada Linn.}

os roftro absque maxillis palpisque.

Rofrum porrectum, inflexum.

Vagina conuoluta, vniualuis, biarticulata: articulo primo craffiori, inae. quali, fecundo longiori, cylindrico, fetas continens fub capitis apice in. ferta.

Sitae tres, aequales, filiformes, apice fubularae, tenues capitis apici infertae.

Labium breue, membranaceum, acutum, integerrimum bafin fetarum tegens.

Antenna fetaceae: articulo primo crafficfimo, reliquis aequalibus fronti infertac.

Larua hexapoda, currens, aptera imagini fimillima.

Puppa hexapoda, currens imagini fimiliima at folis alarum rudimentis.

Victus e fucco plantarum roftro hamiendo. 


\section{CICADA. Iinn.}

Os roftro absque maxillis palpisque.

Roftrum breue, inflexum.

Vagina breuiffima, vniualuis, membra. nacea, cylindrica, obtufa, triarti. culata: articulis fubaequalibus, fecundo fubcraffiori fub fetarum bafs inferta.

Sctac tres porrectae, fubulatae acutae, vagina longiores, inaequales: intermedia longiori, acutiori fupra bafin vaginae infertae.

Labium porrectum, membranacelim, te. nuifimum, acutum bafin fetarum fu. perne tegens.

Antennae teruifimae, fubulatae: articulis vix diftinguendis fub oculis infertae.

Lsrua hexapoda currens imagini fimillima at aptera.

Pupp hexapoda, currens imagini finilli. $\mathrm{ma}$ at folis alarum rudimentis.

Victus omnium e fucco plantarum. 
15.4. CER COPIS. Cicada Linn. Geoff.

O) rostro absque maxillis palpisque.

Roftrum infexum.

Vigina porrecta, vniuluis, obtufa, filperne canaliculata, triarticulata: anrisnlo fecundo maiori, tertio frib. conico, fub fetarum bafi inferta.

Setae tres, fubulatae, acutae in vaginae dorfo latentes, inaequales: intermedia longiori fub capitis apice infertac.

Labium abbreuiatum, membranaceum, truncatum, emarginatum bafin feta. rum tegens.

Antcnate fubulatae, tenuiffimae: articulis vix diftinguendis fub oculis infertae.

Iarua hexapoda, currens, agilis imagini fimillima at aptera.

Puppa hexapoda currens, agilis imagini fimillima at folis alarum rudimentis.

Fitius \& laruae \& puppae \& imaginis o fucco plantarum. 
155. NOTONECTA. Linn. Geoff.

Os roftro absque maxillis palpisque.

Rofirum arcuatum, inflexum.

Vagin porrecta, cornea, vniualuis, obtufiufcula, concaua, triarticulata: articulo fecundo longiffimo, crafo fiori, fetas continens.

Setae tres, fetaceae, fubulatae, acutae, insequales: intermedia longiori ad. bafin vaginae infertae.

Labium rigidum, conicum, corneum, apice membranaceum, acuminatum bafi dilatatum roftri bafin tegens.

Antennac brenes, triarticulatae: articulo primo \& fecundo cylindricis, vltimo breuiore fetaceo, fub oculis infertae.

Larua hexapoda, agilis, natans, imagini fimillima at aptera.

Puppa hexapoda agilis, natans imagini fimillima at folis alarum rudimentis.

Victus omnium e fucco animalculorum aquaricorum. 


\section{भत्र}

156. SIGARA. Notoneta Linn.

Corixa Geoff.

Os porrectum, corneum roftro, absque pal. pis maxillisque.

Roftrum breue, fubinflexum.

Vigina vniualuis, breuis, fubarcuata, conica, acuminata, cornea, con-

- caua, lateribus fpinofa, transuerfe fulcata totum os obtegens.

Sitac tres fetaceae, fubulatae, acutae, diftantes, inaequales: intermedia longiori fub capitis apice infertae.

Labium nullum at vagina capiti adhaeret, os vtrinque tegit.

Antennae breuilfimae, filiformes, triarticulatae: articulis aequalibus fub oculis infertae.

Larua hexapoda, agilis, natans, imagini fimillima, at aptera.

Puppa hexapoda, agilis, natans, imagini fimillima, at folis alarum rudimentis.

Ditus e rapina animalculorum aquatiçopum. 
15\% NEPA. Linn. Hepa Geoff.

Os roftro absque palpis maxillisque.

Rofrum breue, arcuatum, acutum, in. flexum.

Vagina vniualuis, cornea, rotundata, fubulata, acutiufcula, triarticulata: articulo primo breuilíno, fecundo longifimo, tertio tentiori.

Setac tres fubulatae, acutae, inaequales: intermedia longiore acuriore, lateralibus duplo breuioribus, intra vaginam latitantes.

Labium vix vllum, truncatum, haud exfertum.

Antenna breues, palmato-diuifae, fub oculis infertae, in folfula oculorum recondendae.

Larua hexapoda, agilis, natans, imagini fimillima, at aptera.

Puppa hexapoda, agilis, natans, imagini fimilima, at folis alarum rudimentis.

Victus e fucco animalium aquaticorum. 


\section{+สำ}

558. NA VCORIS. Geoff. Nepa Linn.

Os roftro absque palpis maxillisque.

Rofrum breue, inflexum.

- Wagina vniualuis, cornea, rorundata, fubulata, acutiufcula, triarticulara: articulo primo \& fecundo crafliori. bus, textio breuiori, fubulato.

Sitac rres filiformes, vagina longiores, apice fubulatae, acutae, inaeghales: incermedia longiore, acutiore, rigidiore fupra bafin vaginae infertae.

Lnbium corneum, porrectum, apice ro. tundatum, integrum, primum rolti articulum tegers.

Ancmua breuifimae, filiformes, fub ocu.

lis infertae.

Lama hexapoda, agilis, natans, imagini fimillima, at aptera.

puppa hexapoda, agilis, natans, imagini fimillima, at folis alarum rudiments.

Dictus \& laruae \& imaginis \& fucco anima. lium aquaticorum. 
159. A C A NTHIA. Cimex Linn. Geoff.

Os roftro absque palpis maxillisque.

Rofrum elongatum, inflexum.

Vagina vniualuis, recta, cylindrica, cornea, acutiufcula, triarticulata: articulis cylincricis, fubaequalibus; fecundo parum longiori fub capitis apice inferta.

Sctac tres, filiformes, acutae, inaequales: intermedia parum longiore.

Labium nullum.

Antennac porrectae, filiformes, triarticulatae: articulis fubaequalibus, cylindricis ante oculos infertae.

Larua hexapoda, agilis, currens, imagini fimillima, at aptera.

Puppa hexapoda, aogilis, currens imagini fimillima, at folis alarum rudimentis.

Victus \& lartae \& imaginis e fucco animalium plantarumque. 


\section{$18 I$}

\section{CIMEX. Linn. Geoff.}

Os roftro absque palpis maxillisque.

Roptrum elongatum, infexum.

Vagina vniualuis, porrecta, cylindrica, cornea, obtufa, fupra canaliculata, fubtus rotundata, fetas includens, quadrarticulata: articulis inaequalibus; primo craffiori, fecundo lon. gilfimo.

Setae tres connituentes, fubulatae, acutae, fubaequales: intermedia pa. rum longiori.

Labium elongatum longitudine dimidiae fetae, validum, fubularum, annulatum, acuium, fetas fuperne tegens.

Antenuac porrectae, filiformes, quadriarriculatae: articulis cylindricis: primo longiori ante oculos ad latera roftri infertae.

Larua hexapoda, agilis, currens, imagini fimillima, at aptera.

Puppa hexapoda, agilis, currens, imagini fimillima, at folis alarum rudimentis.

Victus e fucco animalium plantarumque. 
16r. REDV VIVS. Cimex Linn. Geoff.

Os roftro absque palpis maxillisque.

Rofrum breue, arcuatum, inflexum.

Vagina cylindrica, cornea, apice feta. cea, fetas continens, biarticulata: articulo primo breuiori, cralfiori, cylindrico, fecundo longiori, fubulato, acutiffimo.

Setac tres conniuentes, fubulatae, acutae, inaequales: intermedia longiore.

Isbium vix vllum, truncatum, nec fupra bafin roftri porrectum.

Antenma porrectae, fetaceae, triarticulatze: articulis cylindricis, vlimo bre. uiori fupra oculos infertae.

Larua hexapoda, agilis, currens, imagini fimillima, at aprera faepiusque quis. quiliis obtecta.

Puppa hexapoda, agilis, currens, imagini fimillima, at folis alarum rudimentis. Victus e fucco animalium. 


\section{जสำ}

I62. PVLEX. I.inn. Geoff.

Os roftro absque palpis maxillisque.

Rofirum elongatum, inflexum.

Vagina cylinarica, biualuis: valuulis aequalibus, rotundatis, canaliculatis, quinquearticulatis: articulis fubaequalibus; vlrimo obtufo.

Seta vnica filiformis, acuta, canaliculata, vagina fublongior.

Laminae duae, ouatae, acuriufculae, vagina multo breuiores ad bafin latera. lem vaginae infertae.

Labium abbreuíatum, rotundatum, aculeis

reflexis ciliatum vix bafin roftri tegens. Aint?nuc porregtae, extrorfim cralfiores, quadriarticulatae: articulis obconicis; vltimo maiori ouato.

Iaru apoda, cylindrica, agilis fpinis duobus, cylindricis, fubcaudalibus. Puppa quiefcens, immobilis, imagini fimillima.

Victus e fucco fanguineque animalium, quisquiliis.

obf. Singulare fane infectum. Differt $a b$ hac claffe vagina biualui, ab Antliatis defectu probofcidis palporumque vaginaque articulata. Ab ambabus vero metamorphofi, qua ad Vlonata accedit.

$$
\mathrm{M}_{4} \quad \text { 163. A. }
$$




\section{A PHIS. Linn. Geoff.}

Os roftro absque palpis maxillisque.

Rofrum elongatum, inflexum.

Vagina porrecta, inuoluta, cylindrica, fetam continens, quinquearticulata: articulo primo maiori, vltimo acuminato.

Seta vnica, tenuiffima, filiformis, apice acuminata, vagina longior, retractilis, intra vaginam latens fupra eius bafin inferta.

Labitim breue, fornicatum, truncatum, integrum.

Antennae porrectae, fetaceae: articulo primo craffiori fronti infertae.

Larua hexapoda, agilis, currens, aptera, imagini fimillima.

Puppa hexapoda, agilis, currens, ima. gini fimillima.

Victus \& laruae \& imaginis e fucco plantarum.

Obf. Aphides aeftate viuiparae, autumno ouiporae. Aliae apterae, aliae alatae absque fexus difcrimine in eadem fpecie.

164. CHER. 


\section{CHERMES. Linn. Geoff.}

Os roftro absque maxillis palpisque.

Rofirum elongatum, infexum vagina pe. ctorali.

Vagina membranacea, cylindrica, ob. tula, triarticulata: articulis fubae. qualibus pectori inter femora antica inferta.

Sctac tres fetaceae, acutac, inacquales: intermedia fublongiori capitis apice infertae intra vaginam pectoralem latentes.

Labium conicum, acutum, compreffum, bafi dilatatum, corneum bafin fetarum fuperne regens.

Antmane bienes, cylindricae, apice feta antennis longiori armata.

Larua hexapoda, aptera, faepe villora, imagini fimillima.

Puppa hexapoda, agilis, imagini fimillima folis tamen alarum rudimentis.

Victius e fucco plantarum. 
165. C OCCVS. Linn. Geoff.

Os roftro abque maxillis palpisque.

Rofrum breue, infexum vagina fetisque pectoralibus.

Vagina cylindrica, membranacea, obtufa, triarticulata: articulis aequalibus pectori inter femora antica in. ferta.

Setae tres fubaequales: intermedia paullo longiore, acutae ad bafin vaginae pectori infertae.

Labium nullum.

Antennae brenes, filiformes, cylindricae, triarticulatae; articulis aeqqualibus; vltimo ouato.

Larua hexapoda, currens, imagini fimil. lima, aptera.

Puppa hexapoda, agilis, imagini fimillima. Victus e fucco plantarum.

O6. Faeminae tardae, apterae, grauidae plan. tis adhaerent fere immobiles. Mares alis duabus erectis, compretis. 


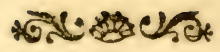

166. THR IPS. Linn. Geofi.

Os rima longitudinali absque palpis maxil. lisque.

Rofrum obfcurum, intra labia rimae re. conditum, haud diftinguendum.

Antensac porrectae, filiformes: articulis feptem; primo maiori, reliquis aequa. libus, vltimo acuto.

Larua hexapoda, aggilis, currens, imagini fimillima, aprera.

Puppa hexapoda, agilis, currens, imagini fimillima folis alarum rudimentis.

Vicbus omnium e polline florum.

Obf. Inftrumenta cibaria huius generis mihi haud rite nota. Ad Ryngota tamen pertinere ob metamorphofin videtur. 


\section{Clajes VIII. \\ A N T L I A T A.}

$O$, hauftello absque mandibulis maxillisque.

Haufcllum plerumque exfertum, vaginatum, cylindricum, intra rimam oris longitudinalem infertum.

Vagina mox vniualuis, mox biualuis fe. tas includens, acuta, inarticulata.

Setae faepius tres, fetaceae, acutae, inaequales: intermedia paullo longiore.

Probofcis quibusdam breuis, membranacea: ftipite cylindrico, retractili; dorfo canaliculato, capitulo bilabiato fub hauftello inferta.

Palpi interdum duo ad bafin probofcidis inferti.

Antennae breues, plerumque cylindricae, faepius feta armatae. 


\section{GENERA Claffis VIII.}

I67. OESTRVS.

168 TIPVLA.

I69. BIBIO.

I70. STRATIOMYS.

I7 I. RHAGIO.

72. SYRPHVS.

173. MVSCA.

174. TABANVS.

175. RHINGIA.

176. ASILVS.

177. CONOPS.

178. STOMOXYS.

179. MYOPA.

180. CVLEX.

181. EMPIS.

182. BOMBYLIVS.

183. HIPPOBOSCA.

184. PEDICVLVS.

185. ACARVS. 
I6\%. OESTRVS. Linn. Geoff.

Os hauftello absque proborcide $\&$ palpis.

Haufellum retractum intralabia veficulofa, tumida, connata, poro paruo, rotundo ad exfertionem hauftelli pertúa.

Vagina membranacea, cylindrica, obtufa, palato inferta feras includens.

Setat tres, membranaceae, flexiles, breues, reflexae, fubaequales labii api. ci infertae.

Palpi nulli。

Antennae breues, filiformes: articulo pri. mo globofo, craffiori fronti infertae.

Larua apoda, breuis, craffa, mollis, annulata, intra animalium corpora latens.

Bupp ouata, apoda, immobilis, cute dura, craffa, brunnea recta.

Eictus laruae e fucco animalium, imaginis vix vllus. 
168. TIP VLA. Linn. Geoff.

Os probofcide, hauftello palpisque.

Probofcis breuifima, vix exferta, membranacea: dorfo canaliculata fetam recipiens; ftipite cylindrico retractili, $\mathrm{ca}$. pitulo bilabiato, aequali.

Hauftllum breue, nudum, absque vagina.

Seta vnica tenuis, longitudine probofcidis, rigida, fetacea, acuta in canali probofcidis recondenda.

Palpi duo porrecti, aequales, incurui, filiformes, multiarticulati: articulis tribus anterioribus craffioribus, abconicis, reliquis breuioribus, cy. lindricis vtrinque ad bafin probo. fcidis inferti.

Antennae filiformes, multiarticulatae: articulo primo craffiori, maiori.

Larua apoda, cylindrica, mollis: capite truncato, dentato.

Puppa immobilis, cylindrica: antice bicornis, poftice dentata.

Victus laruae e radicibus plantarum, imaginis e quisquiliis. 
169. BI BIO. Mufca Linn. Geoff.

Os probofcide, hauftello, palpisque.

Probofcis membranacea, exferta: ftipite cylindrico, reflexo; dorfo canaliculato ad receptionem ferarum, capirulo bilabiato labiis aequalibus.

Hauftellum vagina vniualui, cornea, reflexa, dorfali, apice truncata, obtufa feras fuperne tegente.

Setac tres fetaceae, fubaequales: intermedia rigidiore, lateralibus diltantibus, palpis breuioribus.

Palpi duo porrecti, filiformes, quadriarticulati : articulo fecundo longifimo, quarto fuborbiculato ad bafin lateralem probofcidis inferti.

Antennac breues, cylindricae, approximatae.

Metamorphofis \& Viquus adhuc omnino latent.

$\dot{M} u \int c a$ illucciss antennis longioribus clauatis. 


\section{I7\%. STRATIOMYS. Geoff. Mufca Linn.}

Os probofcide, hauftello palpisque.

Probofcis breuis, exferta: ftipite fubcorneo, cylinárico, recuruo; dorfo ca. naliculato ad receptionem ferae, capitulo membranaceo, incrafiato, bilabiato: labiis aequalibus.

Iauffllum absque vagina.

Sera vnica, valida, porrecta, longitu. dine probofcidis, cylindrica, caua, obrufa, medio dorfali probofcidis inferta in cuius canali dorfali recon. ditur.

Palpi duo breues, fubcapitati, triarticuJati: articulis cylindricis; vltimo craffiori, medio laterali probofcidis inferti.

Antennae porrectae, approximatae, cy. lindricae, acurae: articulo primo ma. jori, reliquis fubaequalibus.

Larta aquacica, apoda, annulata, fubcy. lindrica, poftice attenuata, apice fpiraculo pertufa, barbata.

Puppa immobilis, cylindrica, cute lar. uae indurata recta.

Vicus laruae e animalculis aquaticis, ima. ginis e quisquiliis. 


\section{I7r. RHAGIO. Tusfses him.}

Os probofcide, hauntello palpisque.

Probofcis membranacea, exferta : dorfo canaliculato ad receptionem fetarum, ftipite breuifimo, cylindrico, capituloque dilatato, adfcendente, bilabiato: labiis aequalibus; ciliatis.

Hauftllum breue absque vagina.

Setae tres filiformes, cylindricae, apice acuminatae, probofcide parum breuiores, inaequales: intermedia parum longiori, validiori ad bafin dorfalem probofcidis infertae.

Palpi duo porrecti, longitudine probofcidis, filiformes, pilofi, quinguearticulati: articulo fecundo longiffimo, reliquis breuioribus, tenuioribus bafi laterali probofcidis inferti.

Antennae breues, approximatae, cylindricae, apice acuminatae, fetigerae.

Metamorphofis \& Victus nobis adhuc latent. 
172. SYRPHVS. Mufca Linn. Geoff.

Os probofcide, hauftello atque palpis.

Probofcis porrecta, exferta, membranacea, dorfo ad receptionem fetarum canaliculata: Atipite elongato, cylindrico, capirulo bilabiato: labiis aequalibus, acutis.

Hucuftllum porrectum absque yagina.

Setae quatuor inaequales: laterales paullo breuiores, fubulatae, intermediae inaequales: interiori minore, acutiore, fubulata, exteriori craffiori, fornicata, vaginante, obtufa, bafi dorfali proboficidis infertae.

Palpi duo fetis parum breuiores, filiformes: articulis aequalibus adhaerentes bafi fetarum exteriorum.

Antennae breues: articulo vltimo com. preffo, fetigero.

Larua apoda, annulata, fubcylindrica, poftice attenuata.

Puppa immobilis, ouata cute dura, mem. branacea tecta.

Victus laruae \& imaginis e quisquiliis. 
I73. MVSCA. Linn. Geoff.

Os proborcide, hauntllo atque palpis.

probolcis cxiferta, bafi finca, dorfo cana. liculato ad receptionem ferne: ftipite corneo, porredto, cylindrico, capitulo ouato, veficulofo, bilabiato: labiis aequalibus, acutis.

Hauftellum breue absque vagina.

Seta vnica, probofcide mulco brevior, cylindrica, apice acuminata ipfae fracturae probofcidis interta.

Palpi duo compreffi, porrecti extrorfum craffiores: articulis aequalibus, obfoleris, baf laterali probofcidis inferti.

Antenna breues, incuruae: articulo vltimo compreflo, ferigero.

Larua apoda, annulata, mollis, fubcylindrica, poftice attenuata.

Puppa immobilis, onata, cute dura, co. riacea, tecta.

Victus laruae e cadaueribus, animalculis, ftercore, imaginis e guisquiliis, 
174. TABANVS. Linn. Geoff.

Os probofcide, hauftello atque palpis.

Probojcis recta, exferta, membranacea, dorfo canaliculata ad receprionem hauftelli : ftipite breui, craflo, cylindrico, capitulo ouato, bilabiato; labiis aequalibus.

Hinffellum porrectum, exfertum, longi. tudine probofcidis.

Vagina triualuis, concaua, acuminata, longitudine fetarum canalem probofcidis fuperne \& ad latera claudens.

Setae tres aequales, compreffac, acutac, bafi probofcidis dorfali infertae.

Palpi duo aequales, triarticulati: articulo vltimo craftiori, incuruo, fupofo, ob. tufo ad latera fetarum probofcidi inferti, reliquis breuioribus, pilofis.

Antennae breues, approximatae, cylindri. cae, acutae, feptem articulatae: arti. culo tertio faepius maiori denteque la. terali armato.

Metamorphofis ignota.

Victus imaginis e fanguine animalium haufto. 
175. RHINGIA. Scop. Conops Linn.

Os probofcide, hauftello atque palpis.

Probofcis reeta, porreAta, membranacea, dorfo canaliculato ad fetarum receptionem: ftipite cylindrico, retractili, capituloque ouato, bilabiato: labiis aequalibus.

Hauftllum rectum, porrectum, vagina vniualui, cornea, fornicata, cultrata, conuoluta, apice acuminata fetas probofidemque continente totumque os obtegente.

Setae quatuor fubulatae, acutae, corneae, fubaequales, lateralibus paullo brevioribus acutioribus, intermedia interiori longiori, validiori vaginante exteriorem acutifimam.

Palp duo brenes, filiformes, triarticulati: articulis aequalibus fub apice fetarum lateralium inferti.

Antennae brelles, approximatae, compreffae, extrorfum craffiores, fetigerae.

Mctamorpóofis ignota.

Vidus imaginis \& rapina Antliatorum, Gloffatorum. 


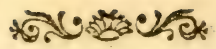

\section{ASILVS. Linn. Geoff. Erax Scop.}

Os hauftello palpisque absque probofcide.

Hauffellum rectum, porrectum.

Vagina biualuis valuulis inaequalibus: inferiori longiori, cornea, cylindrica, bafi gibba, apice obtufa, fiffa fetas continente, fuperior multo breuiore, concana, acuriffima bafin fetarum tegente.

Setae tres porrectae, filiformes, acutae, inaequales: intermedia longiore, validiori, acutiori.

Palpi duo breues, filiformes, pilofi, triarticulati: articulis aequalibus, cylindricis ad bafin lateralem hauftelli inferti.

Antennae breues, approximatae, filiformes: articulis anterioribus longioribus, vltimo fubcraffiori fetaceo acuto.

Metamorphofis ignota.

Victus Imaginis e rapina inprimis antliato. rum, gloffatorum. 
17\%. CONOPS. Iinn. Aflus Geoff.

Os probofcide, haufello atque palpis.

Probafcis porręta, elongata: fipite corneo, cylindrico vlria bafin geniculato, dorfo canaliculato ad receptionem $f \mathrm{c}$ tae, capitulo ouato bilabiato: labiis aequalibus, rotundatis.

Hazfellum vagina vniualui valuula abbre. uiata, concaua, acuminata canalem dorfalem probofcidis claudente.

Seta vnica cylindrica, rigida, acuta longitudine probofcidis, cuius genicu. lo inferta eft.

Palpi duo breuifimi, filiformes, triarticulati: ariculis aequalibus adhaerentes lateribus geniculi probofcidis.

Antenrae approximatae, clauatae: claua acuminata, quadriarticulatae: articulo fecundo longiori.

Metamorploojis ignota.

Victus Imaginis e fusco animalium haufo. 
178. STOMOXYS. Geoff. Conops Linn. Empis Scop.

D) hauftello palpisque absque probofcide.

Hinffellum porrętum, elongatum vitra bafin geniculatum.

Vagina vniualuis, cornea, conuoluta, bafi compreffa, apice cylindrica, ob. tufa, fifla fetas continens.

Sctae duae aequales, filiformes, acutiffimae: fuperiori craffiori inferiorem tenuiorem vaginante.

Palpi duo breues, filiformes, triarticulati: articulis fubaequalibus, cylindricis, bafi hauftelli inferti.

Antennae brenes, approximatae, infexae, apice craffiores, obtufae, fetariae.

Metamorphofis latet.

Vičtus Imaginis e fanguine animalium haufto. 
179. MYOPA. Conops Linn. Afilus Geoff. Sicus Scop.

Os hauftello palpisque absque probofcide.

Hauftellum porrestum, elongatum, bafi medioque geniculatum.

Vagina biualuis:valuulis inaequalibus; inferiori elongata, cylindrica, geniculata, apice obtufa fiffa, fuperiori breuiori, concaua, acuminata.

Seta vnica, rigida, fubulata, tenuis, acuta longitudine valuulae inferioris geniculo vaginae inferta.

Palpi duo breues, pilofi, extrorfum craffiores, triarticulati: articulis fubaequa. libus, hauftelli bafi laterali inferti.

Antennac incuruae, clauatae, fetigerae. Mctamorpbofis omnino adhuc latet.

Victus Imaginis e fucco animalium haufto. 


\section{C V LEX. Linn. Geoff.}

Os hauftello palpisque absque probofcide.

Haufcllum rectum, porrectum.

Vagina vniualuis, elongata, cylindrica, flexilis, inuoluta, apice acuminata, perforata, fetas continens.

Setae quinque fubaequales longitudine vaginae, fubclauatae, apice mucro. natae, acurae.

Palpi duo aequales, filiformes, pilofi, triarticulati: articulis cylindricis; primo longiori, ad latera hauftelli inferti.

Antennae filiformes: articulis plurimis, globofis, breuibus, faepius pectinatis.

Lama aquatica, apoda, thorace incraffa. to, abdomine cylindrico, refpiratorio cylindrico ani.'

Puppa quiefcens, incuruata, ouata refpiratoriis duobus corniformibus dorfi.

Vitus laruae e infectis aquaticis minuriflimis, jmaginis e fanguine Manma. lium haufto.

Obf. Maris palpi porrecti longitudine ro. ftri, faeminae vero abbreuiati. 
18r. EMPIS. Linn. Afilus Scop.

Os probofcide, hauftello palpisque.

Probofcis elongata, exferta, inflexa, hau-

ftelliformis: ftipire cylindrico, tenui, dorfo canaliculato ad receptionem han. ftelli, captulo elongato, oblongo, bilabiato: labiis aequalibus, ciliatis, acuris. Hatuflum probofide breuius, infexum.

Vagina vniualuis, cornca, cylindrica, apice acuminata, fiffa, fetas con. tinens.

Setac tres rigidae, filiformes, apice actr. minatae, inaequales intermedia lon. giori, validiori, lateralibus breuif. fimis tenuioribus.

Palpi duo breues, pilofi, renexi, filiformes triarticulati: articulis fubaequalibus ad probolcidis balin inferti.

Antenna approximatae brenes, filiformes, triarticulatae, articulo primo craffior pilofo, vltimo acuto.

Motamorphofis omnino adhuc latet.

Victus imaginis e fucco animalium, e rapina inprimis antliatorum.

06\%. In quibusdam fpeciebus probofidis capitulum obfolerum. 
182. BOMBYLIVS Linn. Afilus. Geoff.

Os haufello palpisque absque probofcide.

Hauftellum elongatum, rectum, fetaceum.

Vagina biualuis, valuulis inaequalibus: inferiore longiore fetacea, inuoluta, fetas continente, apice acuminata, fiffa, fuperiori porreeta, filiformi, acutiffima.

Setae tres, fubulatae, inaequales, intermedia longitudine vaginae, lateralibus brenioribus, acutis, rigidis.

Palpi duo breues, pilofi, triarticulati: articulis aequalibus, cylindricis bafi la. terali hauftelli inferti.

Antennae breues, approximatae, filiformes, apice acuminatae.

Metamorphofis omnino adhuc latet.

Victus imaginis e nectare florum. 
I83. HIPPOBOSCA. Linn. Geoff.

Os hauftello absque probofcide \& palpis.

Hauftellum breue, rectum, porrectum, cylindricum, rigidum.

Vagina biualuis, valunlis aequalibus, fimicylindricis, ciliatis, obtufis, emarginatis fetam continentibus.

Seta vnica, fubulata, rigida longitudine vaginae.

Antennae approximatae, breuifimae, filiformes, biarticulatae articulo primo breniffimo, fecundo tenuiffimo.

Metamorphofis latet.

Victus imaginis e fanguine animalium haufto. 
184. PEDICVLVS. Linn. Geoff.

Os hauftello absque probofcide \& palpis.

Haufellum breue, cylindricum, retracti. le, rectum, rigidum.

Vagina biualuis, cylindrica: valuulis aequalibus femicylindricis, annulatis, truncatis, obtufis, fetam continentibus.

Scta vnica, recurua, fubulata, rigida, acutiffima longitudine vaginae.

Antennae breues, faepius moniliformes: articulis aequalibus.

Larua hexapoda, agilis, currens, imagini fimillima.

Puppa hexapoda, agilis, currens, imagini fimillima.

Victus \& laruac \& imaginis e fucco animalium haufto. 
185. ACARVS. Linn. Geof.

Os hauftello palpisque absque probofcide.

Haufellum breue, porrectum, rectum, cylindricum, rigidum.

Vagina biualuis valuulis aequalibus, emi . cylindricis, obtufis, horizontalibus: fuperiore ad bafin fira: laciniis aequalitus, cylindricis, inferiore planiore.

Seta.

Palpi duo comprefi, aequales, porrecti, longitudine hauftelli, obtuli, rigidi, miarticulati: articulis àequalibus, ad bafin lateralem haufelli inferti.

Antenne filiformes, compreffi: articulis fubaequalibus, pedes aemulantes.

Larua hexapoda, agilis, currens, imagini fimillima.

Puppa hexapoda, agilis, currens, imagini fimillima.

Victus \& laruae \& imaginis e fucco animalium haufto.

Ob/.I. Acarus Ricinus antennas clauatas gerit. Obf. II. Pediculus \& Acarus metamorphofi ad Ryngota accedunt.

MANTIS. 


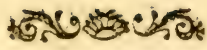

\section{9}

\section{$M A N T I S S A$}

\section{jpecierum nuper detectarum}

IOx.- 2. GCARABAEVS exfcutellatus, tho Vitu-

race quadridentaro, occipite cornubus lus duobus erectis breuibus, corpore atro.

Habitat in Aufria Dr. Schulz.

Magnitudo \& ftatura S. Vaccae. Clypeus rotundatus ater medio linea transuerfa ele. uata, pof́rice corniculis duobus eleuatis brevibus. Thorax antice quadridentatus denticuiis aequalibus breuibus, poftice rotundatus absque fcutello. Elytra breuia, atra: Pedes arri.

104--5 SCARABAEVS exfutellatus, tho fictrorace inermi rugofo, capitis cornu eredtofus breuifimo, elyuris teftaceis nigro fcabris. Irabitar Surinami Dr. Schulz.

Statura omnino S. nuchicornis at paulio maior. Caput cupreo aeneum clypeo antice truncato poftice cornu breui, erecto. Thorax cblcurus, variolofus maigine pallidiore. Elytra tefracea punctis nigris eleua. tis per frias difpofitis, fcabra. Corpus nigricans. Pedes pallide teftacei femoribus bafi tibiisque apice nigris.

6.7. MELOLONTHA oblonga glabra ni- oblon gra, elytris obfolete ftriatis.

Scar abreus oblongus Scop. carn. Ig.

$$
\text { O Habi. }
$$


Habitat in Germania.

Minor M. vulgari tora nigra, glabra. Ocu. li tefacei. Elytra Atriis per paria approximatis, fubpunctatis, obfoletis.

varia- $35 \cdots 36$. MELOLONTHA atra, elytris aubilis ro nitentibus, antennis pedibusque te. ftaceis.

Habitat in America boreali.

Antennae trilamellatae pallide teftaceae. Caput \& thorax atra, immaculata. Elytra ftriata, atra tomento aureo fere obfoleto fplendentia. Pedes teftacei.

Eandem magna in copia in horto Kilonienfi fub terra e ligno putrido nata degentem vidimus, at elytra haud auro nitentia fed tomento cinereo tecta.

Variat interdum colore rufo.

Lynx 39-40. MELOLONTHA nigra, hirta, ely. trorum margine aureo.

Habitat ad Cap. B. Spei. Dom. Lee.

Starura \& magnitudo M. Vrfus, cuius forte mera varietas. Differt tantum elytrorum margine exteriori aureo, nitido.

crimita 39 40. MEI.OLON'THA hirta fupra viridis fubtus nigra.

Habitat ad Cap. B. fpei. Dom. Lee.

Affinis praecedenti tota pilis longioribus nigris hirta at fupra viridis, nitens.

$20 \cdots 2 I$. 
20.2r. CETONIA nigra, thorace lineis trilitribus, elytris fafcia flexuofa fcutelloque neata albis.

Habitat ad Cap. B. Spei. Dom. Lee.

Media. Caput nigrum linea longitudinali flaua. Clypeus emarginatus. Thorax niger linea dorfali lateralibusque flauis. Punctum nigrum vtrinque in margine. Scutellum triangulum flaumm. Elytra fubftriata, nigra fafcia flexuofa, abbreuiara, flana. Abdomen nigrum ano fafcia flexuofa flaua. Pe. des nigri flamo variegati.

I-2. BOSTRICHVS piceus, elytris apice fauifpinofo dentatis, antennis luteis. Habirat in Ameriça boreali.

Medus totus cylindricus. Caput nigricans antennis flauis claua compreffa. Thorax cylindricus. Elytra fubftriata apice vix retufa, denticulata denticulo poftico elongato in fpinam validam, acutiflimam. Pedes vti corpus fubrus brunnei.

3-4 BOSTRICHVS nigricans, elytris glaw policis obtufufculis. Dermeftes poligrapbus Linn. Syft. Nat. 2. phus 562. 10. Fn. Sv. 420.

Habitat in Europa boreali fubarborum corticibus.

\%. BOSTRICHVS elytris integris glaber mimu. ater immaculatus.

$$
\text { O } \& \text { Habi- }
$$


Habitat Kiloniae in Boleto verficolore.

Corpus minimum, atrum, glabrum, im. maculatum. Antennae clau fulida, obtura. Elytra laenia, glabra, integra. Pedes nigri. fani- 3-4. ANOBIVM villólum nigrum, antenpes nis pedibusque teftaceis.

Habitat Hamburgi Dr. Schulz.

Magnitudo \& ftatura A. pertinacis. An tennae filiformes, teftaceac. Caput inflexum. Thorax minus quam in reliquis fpeciebus rotundatus, villofus, niger. Elytra villofa, nigra. Pedes omnes teftacei.

gibbo. 2-3. BRVCHVS thorace gibbofo cupreus $_{3}$ fus elytris nigris tuberculato fpinofis. Habitar in America boreali.

Caput reconditum fubcupreum, antennis filiformibus fauis. Thoras cupreus, tuberculatus, in medio gibborus gibbere canaliculato. Elytra nigra tuberculis plurimis eleuatis, obtufis, fubfpinofis; poftice retu. fa. Pedes nigricantes.

margi.5 - 6. BRVCHVS niger, elytris cinereis mamalis culis tribus nigris margine connexis. Habitat in Germania de Hattorf.

Antennae porredtae, nigrae. Caput \& thorax atra puncto paruo cinereo ad bafin thoracis. Elytra laevia, cinerea, macula ad bafin, alcera maiori in medio marginis 


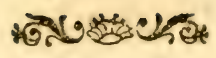

exterioris, tertiaque in apice elytri nigris. Difcus elytrorum immacularus. Anus promincns, cinereus. Pedes nigri.

x-2. ELOPHORVS grifeus, thorace ely-mbilus trisque fulcato rugotis.

Habitat in Holfatiae aquofis Dr. Schulz.

Statma omnino E. aguatici at paullo minor. Antennae clauatae. Thorax grifeus falcis quinque exaratus. Elytra fulcata ab. fure grifea. Pedes concolores.

3. TRITOMA? nigra elyoris pedibusquedabia teftaceis.

HabitatKiloniaeHolfatorumDom.Lund.

Parua huius generis videtur, quum antennae omnino perfoliatae. Caput \& thorax nigra cinereo tomentofa. Elytra poftice acuta tefaces

ฯ. IPS. atra, elytris fafiis duabus rufis: fafcia. anteriore nigro maculata.

Habitar in America boreali.

Magna, oblonga. Antennae porrełae, arrac articulis tribus vltimis perfoliatis. $\mathrm{Ca}$ put \& thorax arra, nirida, immaculata. Coleoptra atra fafciis duabus dentatis, rufis, anteriore bafeos maculis tribus atris; interrietla maiore, communi. Pedes atri.

2. IPS nigra, elytris punctis duobas ferru- 4 pucrineis.
$\mathrm{O}_{3}$
Trito.

Pulata 
Tritoma 4 puftulata Syft. Ent. 69. 3.

Habitar in Europae ligno humido.

Varieratem quadruplo maiorem vix ta. men diftinctam ex America boreali habui.

picea 3. IPS picea, elytris nigris: bafi fafciaque poftica ferrugineis.

Habitat in Germania de Hattorf.

Minor I. quadripufulata. Caput nigricans, ore antennisque piceis. Thorax vtrinque puncto bafeos impreffus. Elytra laeuia, nigra bafi fafciaque poftica lunata ferru. gineis. Corpus \& pedes picei.

4 gut 4 . IPS atra nitida; elyrris maculis duabus ista albis: anteriore linuata.

Habitat Kiluniae Holfatorum. Dom. de Seheftedt.

Starura praecedentium, at multo minor. Caput \& thorax atra, nitida, immaculata. Elyrra laeuia, atra maculis duabus transuer. fis margines haud attingentibus albis; ante. riore bilinuara. Pedes nigri.

cress 5. IPS nigra, thorace rugofo, elytris fria. ta tis: maculis duabus rubris.

Tritama crenatu Sylt. Ent. 69. 4. Habitat in Sveciae lignis.

nufo. 6. IPS glabra atra, fronte pedibus maculisfrons que duabus elytrorum ferrugineis.

Tritoma ruffifrons Syftem. Ent. 69. 5. Habitat in Anglia. 


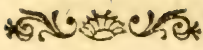

3-4. HISPA antennis pectinatis, thorace ely bicos. tricque viridi aeneis, capire bicorni. Habitat in America boreali Dom. Yeats.

Corpus paruum, piceum. Antennae bre. ues recundum roram longirudinem pectina. tae fufcre bafi ferrugineae. Caput viridiaeneum corniculis duobus breubus, erectis, acuris. Pedes ferruginei.

7-8. SILPHA arra, thoracis margine palii. do, elytris fufcis.

Habitat in America boreali Dom. Lee.

Magnitudo S. rugofae. Caput atrum, ru. golum. Thorax ater margine finuato, pallido. Scutellum atrum. Elytra fufca ftriis tribus eleuaris laevibus. Pedes nigri.

9-- I0. SILPHA atra, elytris punctatis: li-lunat neis elcuatis tribus laeuibus, thorace transuerfo emarginato.

Habirat in Autria Dr. Schulz.

Maior S. arrata. Caput atrum. Thorax laritudine elyrrorum, rransuerfus, antice valde emarginatus ater punctis ducbus paruis, dorfalibus, nitidulis. Elyrra valde punctata ftriisque tribus eleuatis. Pedes atri. 1-2. NITIDVLA ouata nigra obfcura, pe obfer.

dibus piceis.

Habitat in Germania de Hattorf.

Statura omnino N. 2 puftulatae at paullo minor. Corpus fupra obfcure nigrum mi$\mathrm{O}_{4}$ nime 
nime nitidum. Elytra lacuia, immaculata. Pedes picei.

bicolor 3-4. IITIDVLA fermginea, elytris nigris: facia baíeos punciogue apicis ferrugineis. Habitat in armis Kilonienfibus Dorr. Lund.

Magnitudo N. ferrugineae. Antennae rufae clana folida. Capur ce thorax ferruginea, immaculata. Myrra glabra, nigra falcia lata bafeos punciogue veríus apicem ferrugineis. Abdomen fufcum. Pedes terruginei.

ho: 4-5. NITIDVLA nigra, elytris apice fere morm. rugineŕs.

boida. lis.

Habirat Hamburgi Dr. Schulz.

Staruma N. Colon at duplo minor. Tho. rax marginatus niger margine ferrugineo. Elytra laeuia, nigua apice late ferruginea. Pedes nigri.

wifa- 5.6 COCCINELLA coleoperis rubris: fas friata fcia media atra.

Habitat Hamburgi Dr. Schulz.

Media. Caput \& thorax atra, immaculata. Elyrra rubra fuccia media lata, atra, quae futuram tamen haud attingit \& ad marginem punctis duobus paruis terminatur, Lincola parua obliqua nigra adfcurellum. Corpus arrum. 
7.8. COCCINELLA coleoptris luteis fa.fexuo.

f́ia Hexuofa punctisque duobus nigris. fa Habitat Hamburgi Dr. Schulz.

Corpus atrum, immaculatum. Thorax ater macula laterali apicis albicła. Elytra litea puncto bafeos orbiculato, fafcia in medio flexuofa \& macula apicis transuerfa, clauata nigris.

33-14. COCCINELLA oblonga, colcoptris7 ma rubris: punctis nigris feptem; communiculat trilobo.

Habitat in Germaniae plantis Dr. Schulz:

Magnitudo \& ftatura oblonga C. I 3 punctacae. Caput atrum. Thorax lacuis, ater margine antico \& laterali albo. Elytra ru. bra punctis nigris tribus primo bafeos ro. tundato, fecundo in medio transuerfo, tertio verfus apicem rotundato. Punctum denique commune trilobum. Corpus nigrum.

38-39. COCCINELLA colcoptris luteis: $15 \mathrm{gta}$. pungtis guindecim albis; communi in tata inedio obfoleto.

Coccinella coleoprris rubris punetis quatuordecim limboque albis. Geoff. Inf.

I. $32 \%$. 14 .

Habitat in Germania.

Corpus mainfculum, rotundatum. Tho rax luteus margine laterali albo puncto meOs dio 
dio luteo. Macula obfoleta albida ad bafin thoracis. Elytra lutea punctis feptem rotundatis albis 2. 2. 2. I. Punctum commune fere obfoletum in medio dorfi.

bae- 43-44. COCCINELLA coleoptris atris apimorr. ce rubris: faficia nigra.

boida. lis Habitat Hamburgi Dr. Schuiz.

Corpus rotundatum. Caput ob.cure rufum. Thorax rufus interdum bafi, interdum punctis tribus bafeos nigris. Elytra atra apice rufa fafcia nigra, quae tamen futuram haud attingit.

Variat frequens puncto in medio dorfi communi, fanguineo.

varia 45 - 46 COCCINEL.LA oblonga, coleoptris bilis

nigris: punctis duobus rubris orbiculatis fubmarginalibus.

Habitat Hamburgi Dr. Schulz.

Differt manifefte a C. bipuftulata. Corpus oblongum, immarginatum. Caput album. Thorax ater margine albo. Elytra laeuia atra puncto rubro.

Variat iunior elytris luteis puncto flaue. fcente, at aetate elytra nigra \& puncta rubra euadunt.

12 p 11.490 . COCCINELLA coleoptris nigris: ftulata punctis duodecim albis; exterioribus margine connexis. 


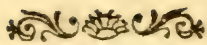

Habitat Hamburgi Dr. Schulz.

Media. Caput albidum puncto frontali nigro. Thorax ater margine antico \& laterali pallido. Elytra atra punctis fex albis 2. 3. r. tribus exterioribus margine connatis. Punctum vitinum lunarum. 7. 8. CASSIDA brunnea, elytris flauis: fu futura-
turis brunneis.

Habitat ad Cap. B. Spei. Dom. Lee.

Magna. Antennae brunneae. Clypeus porrectus, integer, brunneus. Elytra lata, flaua futura dorfali \& bafeos brunneis. Corpus brunneum.

I I-I2. CASSIDA teftacea, elytris anguloperfo. antico fpinofo bafique perforatis.

C. Jida perforata Pall. Spicil. fafc. IX. p. 3 . tab. I. fig. I.

Habitat in America meridionali.

6.-7: CHRYSOMELA atra, thoracis mar- ${ }_{3}$ lineagine flauo puncto nigro, elytris flauis: $t a$ futura vittajue nigris.

Habitat in Aufria Dr. Schulz.

Afinis C. futurali. Caput nigrum fronte flaua. Thorax ater margine larerali flauo puncto medio nigro. Scutellum atrum. Elytra flaua futura nigra, quae tamen fcutellum haud attingit. Vitta lata nigra in medio 


\section{0}

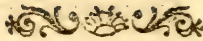

medio elytrorum, quae nec ad bafin nec ad apicem excurit. Corpus \& pedes atra plantis f.Ifcis.

do:5. 6.. . CHRYSOMFLA atra, thoracis marlis

gine reltaceo puncto nigro, elytris tefta.

ceis: furura abbreuiata atra.

Habitat in Aufria Dr. Schulz.

Affuis viderm praecedenti at minor. Elytra tefacea fola futura atra, quae bafin haud atringit. Anrennae \& pedes ningri. Scutellum atrum.

4 ma.59-60. CHRYSOMELA obionga, thora. culata ce teftaceo immaculato, elytris teftaceis maculis duabus nigris.

Habitat in nemoribus Kilonienfibus Dom. Lund.

Magnitudo \& Atatura C. Alni. Caput te. ftaceum bafi inter oculos nigrum. Thorax rotundatus, teftaceus, immaculatus. Elytra lacuia, nirida, teftacea maculis duabus magnis nigris, alrera bafeos rotundata, altera pone medium transuer fali fere faform conftituente. Abdomen arrum. Pedesteftacei.

pub- I - I2. CRYPTOCEPHA VS thorace elyfrems trisque obfcure aeneis pubeficentibus. Habitat in America boreali.

Pauilo minor C. longrimano. Antennae porrectae, filiformes, cincreae. Caput, thorax 
tharax \& elytra obfcure aenea tomento cinereo breui denfo pubefientia. Scutellum nigrum. Corpus fubtus \& pedes cinera. fcentia.

17-18. CRYPTOCEPHALVS capite tho-nigria raceque rufis, elytris pallidis, pedibus ${ }^{\text {pes }}$ apice nigris.

Habitat in America.

Magnitudo \& ftatura C. Coryli. Capué rufum, immaculatum antennis nigris bafi rufis. Thorax rufus, nitidus, immaculatus. Scutellum nigrum. Elytra pallida futura margineque nigricantibus. Abdomen pallidum ano teffaceo. Pedes teltacei apice nigri.

23-24, CRYPTOCEPHALVS ater, elytris qua-

flauis: vitta abbreuiata media atra.

Habitat in Germania. de Hattorf.

Magnitudo C. Moraei. Corpus totum atrum, nitidum. Elytra laeuia, flaua, vitta media lata, atra, quae apicem tamen haud attingit, fed ante apicem reflectirur ad fututam.

32-33. CRYPTOCEPHALVS thorace ful pufillus

uo, elytris teftaceis inigro-maculatis.

Habitat Hamburgi Dr. Schulz.

Paruus duplo minor C. Pini. Caput fuluum antennis pallidis oculisque nigris. Thorax 
Thorax fuluus, immaculatus: Elytra fub. Itriata, teftacea punctis duobus bareos fa. fciaque poftica nigris. Corpus niorrum. Pedes pallidi.

Variant interdum maculae elytrorum, mox confluunt, mox omnino defunt.

24 purs. I I - I 2. CRIOCERIS thorace cylindrico ful. Etata uo punctis quinque nigris, elytris flauis punctis feptem.

Aitelabus if punctatus Scop. Ent. I 16.

Habitat in Germania.

Magnirudo \& fatura C. I 2 punctarae. Ca. put fuluum, ore, antennis punctoque verticali nigris. Thorax fuluus punctis quin. que nigris. 4 . Elytra palidiora punctis 1.2. 2. 1. 1., quorum fextum maius, rotun. datum, feptimum terminale. Abdomen $\&$ femora fulua.

ailara-5-6. EROTYLVS ater, thorace elytrisque zus

ferrugineis.

Habitat ad Cap. Bon. Sp. Lee.

Magnus. Antennae fufcae bafi ferrugineae. Thorax ferrugineus, immaculatus. Elytra corpore multo latiora, lacuia, ferruginea, immaculata. Corpus \& pedes atra.

enfus 9. EROTYLVS oblongus niger, pedibus piceis.

Habitat Kiliae Holfatorum. Dom. Lund. Praede 
Praecedentibus minor. Antennae filifor. mes, nigrae primo articulo ferrugineo. $\mathrm{Ca}$ pur \& thorax nigra, obfcura. Elytra ftriata, nigra, immaculata. Pedes picei geniculis nigris.

5.6 LAGRIA cyanea capite thoraceque ruffo rufis. froms

Habitat in America boreali.

Antennae longitudine fere corporis fufcae apice rufefcentes. Caput rufum oculis magnis nigris. Palpi antici exferti, fecuriformes, fufci claua rufa. Thorax cylindricus, rufus. Elytra ftriata, cyanea. Pedes cyanei femoribus anticis rufis.

6-7. CVRCVLIO longiroftris rufo nigro varius que varius, roftro apice nigro. Habitat ad Cap. B. S. Dom. Lee.

Antennae fractae, capitatac. Roftrum elongatum, cylindricum, ferrugineum apice nigrum. Thorax ferrugineus lineis tribus nigris: lateralibus abbreuiatis. Pectus nigrum lineis tribus ferrugineis. Elytra ftriata, nigra lineolis aliquot ferrugineis. Pedes nigri femoribus ferrugineis annulo nigro.

22-23. CVRCVLIO longiroftris ater, an- Pruni tennis ferrugineis, thorace bituberculato.

Curculio Pruni Linn. Syft. Nat. 2.507. I 2. Habi。 
Habitat in Cerafi foliis.

Valde diftinctus tuberculo vtrinque eles tuato thoracis. Elytra ftriata.

Cam- 22-23. CVRCVLIO longiroftris niger onapambice tus, elytris flriatis obtufis.

Curculio Campanulae Linn. Sytt. Nat. 2. 506. 7 .

Habitar in Campanulae rotundifoliae pericarpis.

Magnirudo \& fatura C. acriduli. Corpus totum nigrum poftice valde obrufum.

saria. $24-25$. CVRCVLIO longiroftris fubtefta. bilis ceus, thorace viridi-lineato, roftro apice fufco.

Habitat Hamburgi Dr. Schulz.

Medius. Caput pallide teftaceum roftro apice fufco. Antennae rufefcentes claua cinerea. Thorax obfcurus lineis tribus diftinctis viridibus. Elytra pallide rufefcentia polline vrrefcenti adfperfa. Pedes rufi.

Sifym. 26-27. CVRCVLIO longiroftris albo fit. brii fcoque varitis, elytris puncto bafeos ele. uiato atro, roftro nigro.

Habitat Kiliae in Sifymbrio amphibio lectus d. xx. Maj.

Paruus. Roftrum elongatum, nigruh. Antennae fufcae, clauatae. Thorax albus macula 
mactila fufca, in qua linea dorfalis alba. Elytra albo fufceque varia $\&$ ad bafin puneto paruo oblongo atro. Corpus fubtus ábidum. Pedes mutici.

3I-32. CVRCVLIO longiroftris niger, ely - $f a$ tris fafciis duabus cinereis: bafeos maio-fiatus re vadata.

Habitat Kiliae Dom. Lund. Noruagus.

Paruus. Rofium elongatum, cylindricum, atrum. Antennae clauatae claua folida, cinerafcente. Thorax rotundatus ater, $l i$ nea dorfali paullo pallidiori. Scutellum cinereum. Elytra ftriata fafciis duabus vndatis cinereis vix marginem exteriorem attin. gentibus, quarum anterior in primis ad fururam maior, altera in medio multo angufior. Pedes nigri, fimplices.

36-37. CVRCVLIO ater, thoracis punetis Crux duobus bafeos, elytrorum futura pundts. gue vaigis albis.

Habitat Hamburgi.

Statura \& magnitudo C. fururalis. Ca. put atrum, immaculatum roftro incuruo. Thorax rotundatus, ater vtrinque puncto oblongo bafeos albo. Scutellum album. Elytra friata nigra, futura bafeos punctisque plurimis vagis albis. Pedes nigri. Cor. pus fubtus albidum.

49-50. CVRCVLIO longiroftris ater, tho-Atrirace nitido, elytris friatis obtufis. plicis

Habi. 
Habitat in Atriplice littorali Florae Kilonienfis Dom. Lund. Norwegienís. Statura \& magnitudo C. linearis, cui val. de affinis. Differt tamen antennis apice haud ferrugineis, roftro tenui bail haud attenuato, thoraceque minus punctato, nitido.

raucus I08-9. CVRCVLIO brenirontris nigricans, elytris grifeis cinereo-punctatis.

Habitat in Germania Dom. de Hattorf. Medius. Antennae medio fractae apice clauatae. Roftrum breue, craffum, fubplanum. Thorax nigricans punctis eleuatis paruis fcaber. Elytra friata friis crenaris, grifea punctis plurimis cinereis fparfis irrorata. Pedes picei femoribus muticis.

bifpi II2-I3. CVRCVLIO breuiroftris fulcus, dulus thorace cinereo lineato, elytris hifpidis punctis obferrioribus frriatis.

Habitat Kiliae in plantis aquaticis Dom. Lund.

Paruus ftatura C. lineati. Antennae cla. uatae articulo primo longiori. Thorax punctatus, fuicus lineis tribus longitudinalious cinereis: media tenuiore. Punctum vtrin. que laterale cinereum. Elytra fufca pilis al. bis erectis hifpida punctisque obfcurioribus ftriata, interiectis pilis albis, qui quafi lineas e maculis nigris albisque conftitumnt. Pe. des nigricantes. 


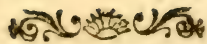

r22-23. CVRCVLIO breuiroftris, thora-fictice elytrisque variolofis nigris albo pun-cus

ctatis.

Habitat ad Cap. B. Spei.

Paruus. Caput nigrum, immaculatum. Thorax variololus, ater, albo punctatus. Elytra variolofa, atra, albo punctata \& verfus apicem fafcia alba. Pedes \& corpus atra. 123-24. CVRCVLIO breuiroftris ater, fuccinelytris margine lineolisque duabus albis. Etus Habitat ad Cap. Bon. Spei. Dom. Lee.

Corpus magnum, oblongum. Caput fulcarum, atrum, immaculatum. Thorax rotundatus, punctatus, ater linea laterali alba. Elytra poftice gibba, punctata, atra margine lineolisque duabus albis, altera a bafi ad medium, altera a medio marginis verfus apicem dueta, reflexa. Pedes atri, fimplices. I25-26. CVRCVLIO breuiroftris grifeus, hiftriothorace lineis lateralibus fuluis, elytrismas albis: maculis atris fuluisque.

Habitat ad Cap. Bon. Spei. Dom. Lee.

Corpus magnum. Caput grifeum roftro craffiuf́culo thorace breuiore. Antennae medio fractae. Thorax dorfo grifeo linea laterali lata fulua margineque albido. Elytra alba maculis plurimis eleuatis nigris duabusque oblongis fuluis. Abdomen albidum vtrinque linea longitudinali punctorum nigrorum. Pedes einerei, inermes.

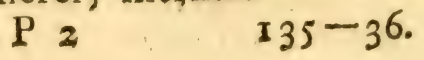


feala. $135-36$. CVRCVLIO breniroftris, tho. race fjinolo inaequali ater, elytris ftriis rufis denticulatis.

Habitat ad Cap. Bon. Spei. Dr. Schulz.

Magnitudo \& itatura C. barbari. Roftrum crafium, breae, obrufun, planum. Antemnae obtufae \& fere truncatae. Thorax acute fpinofus fulcis tribus punctisque quatuor bafeos profundis impreffus. Elycra connata, laevia, atra ftris octo fecundum totam longitudinem denticulatis rufis. Pedes atri femoribus fimplicibus.

4 Spi. I 39-40. CVRCVLIO breuiroftris albidus, nogus elytris 4 fpinofis, roftro fufco. Habitat ad Cap. B. Spei.

Minor. Roftrum breuifimum, canaliculatum, fufcum. Thorax rotundatus, albidus, immaculatus dorfo plano. Elytra albida fpinis quatıor erectis, validis, folitaria verfus bafin, duabus in medio \& quarta verfus apicem folitaria. Pedes albi, fimplices. mailus $145-46$. CVRCVLIO breuiroftris, femoribus dentatis grifeus, elytris punctis ob. fcurioribus numerofis.

$$
\text { Habitat Hamburgi Dr. Schulz. }
$$

Corpus magnitudine \& ftatura C.Liguftci. Antennae clauatae claua acuminata, nigrae. Caput \& thorax grifea, immaculata. Elytra punctis friata punetisque plurimis vagis diftinctis obfcurioribus. Femora poftica anualo albo. 
1.46-47. CVRCVLIO breuiroftris, femo. gem. ribus dentatis ater, elytris punctis viri-matus dibus.

Circulio gemmatus Scop. Ent. 90.

Curculio gemmatus Naturforfcher Sect. 6. tab. 4 .

Habitat in Europa.

I 46 - 47. CVRCVLIO breuiroftris, femo-picipes.

ribus dentatis grifeus, elytris nebulofis, femoribus pofticis rufis.

Habitat Kiliae Dom. Lund.

Minor C. nebulofo. Antennae fractae, clawatac, clana obtufiufcula. Rofrum craffum, brene, obtufum, apice niogrum. Thorax grifeus punctis eleuatis globofis minutiffimis. Elytra nebulofa punctis eleuatis ftriata. Pedes itidem grifei femoribus inprimis pofticis rufis.

2-3. ATTELABVS ater, elyzris macula 2pmfu. balens rufa.

Habitat in America boreali Dom. Yeats. Staitura \& magnitudo A. curculionoidis. Corpits atrum, nitidum, elytris macula magna bacos rufa. Pedes atri femoribus an. ricis intus vnidentatis.

I-2. CIERVS rufus, elytris nigris fafcia dubius duplici alba bafique rufis.

Fabitat in America boreali Dom. Yeats. Ninis afunis C. formicario. Antennae $\mathrm{P}_{3}$ breues 


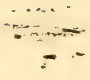

\section{0}

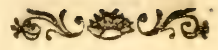

breues, moniliformes. Caput rufum. Thorax poftice anguftatus, rufus. Elytra nigra bafi rufa fafciisque duabus vndatis albis.

ichneu-I-2. CLERVS capite thoraceque rufis, shoneus elytris nigris: fafcia media rufa ftrigaque apicis alba.

Habitat in America feptentrionali Dom. Yeats.

Statura omnino C. formicarii. Caput rufum antennis fulcis. Thorax gibbus, rufus, immaculatus, poftice anguftatus. Elytra

- bafi fubferruginea, tunc nigra, in medio fafcia lata, rufa apice nigra ftriga poftica alba. Pectus nigrum. Abdomen rufum. Pedes fufci.

pun. 2I -22. LAMIA thorace fpinofo ater, ely. ctator tris albo punctatis, antennis longis.

Habitat in China Muf. Dr. Fothergill. Antennae corpore longiores, nigrae articulis bafi pallidis. Thorax rotundatus, fpinofus, ater. Elytra atra, albo-punctata, bafi fcabra. Pedes atri.

arifpa 2I-22. LAMIA thorace fpinofo, elytris lineis tribus eleuatis ferrulatis, antennis mediocribus, corpore cinereo.

Habitat ad Cap. Bon. Spei Dom. L.ee. Corpus magnitudine L. triftis totum cinereum. Antennae corpore breuiores. Thorax inaequalis, acute fpinofus. Elytra ci-

nerea 
nerea lineis tribus eleuatis, acute ferrulatis. Pedes cinerei.

29-30. L. thorace fpinofo tuberculato ci-Spengnerea, elytris fcabris: maculis duabusleri lateralibus atris.

Habitat in America meridionali. Spengler.

Duplo minor L. Rubo. Aniennae corpore longiores, cinereae articulornm apicibus fufcis. Thorax rotundatus, cinerens, vtrinque obrufe fpinofus tubcrculisque tribus dorfalibus. Elytra punctis elcuatis fcabra, cinerea maculis duabus marginalibus atris, quorum pofterior frigam emittit. Elytra apice acuminata. Pedes nebuloti.

7-8. SAPERDA grifea, virtis tribus den-vittatatis albis, antennis longis.

Habitat in India Dr. Fotheroill.

Antennae cinereae corpore duplo longio. res. Caput grifeum linea laterali alba, dorfali ferruginea. Thorax rotundatus, muticus lineis tribus albis. Coleoptragrifea, lincis lateralibus futuralique dentatis albis. Corpus grifeum.

4-5. CALLIDIVM thorace verrucofo ni- acumigricante, elytris acuminatis viricibus: natum furura cyanea.

Habitar ad Cap. B. Spei Dom. Lee.

Minor C. violaceo. Caput fufcum. An-

P 4

tenna 
tennae longitudine corporis, fufcae articulis apice fpinofis. Thorax rotundatus, niger tuberculis plurimis eleuaris. Elytra viridia, nitida futura cyanea, apice acuminata. Pe. des nigri femoribus clauatis claua rubra.

m/ji- 4-5. CALLIDIVM thorace verucofo, nicum grum, elytris taflaccis macula media apiceque nigris.

\section{Habitat in Rufia Dom. Lee.}

Statura C. Bajuli at triplo minus. Capue nigrum, immacuhrmm aatenas longirudine fere corporis. Thorax niger verrucolius. Elyra lacuia, tefacea macula media apiceque nigris. Pecies nigri, mutici.

mipes is - 16. CALLIDIVM thorace laeni nitido, elymis violaceis, tibiis rufis, antennis breuibus.

Habirat in Germania Dom. de Hattorf. Corpus paruma. Antennae brewes, fufeas bafi ferrugineac. Caput \& thorox lae. uia, nitida, violacca. Elyria laeuia, violacea, immaculata. Abdomen seneum, ni. tidum. Pedes violacei tibiis rufis. Femo. ra poftica clauara bafi ferruginea.

wam 2I-22. CALLIDIVA thorace rotundato rufo, elytris nigris ban rufis: faftis dua. bus albis.

Habitat in America boreali Dom. Yeats. Corpus C. vndato minus. Antennse nigrae 


\section{+5.4.}

grae longitudine corporis. Caput rufum. Thorax rotundatus, inermis, rufus. Ely. tra lacuia, nigra bafi rufa fafciis duabus albis. Pedes nigri femoribus clauaris, rufis. Abdomen atrum.

I-2. LEPTVRA nigra, elytris teftaccisliwida immaculatis, pedibus nigris.

Habitat in floribus Chilonii Dom. Lund. Statura \& fumma affinitas L. melanurae. Caput, Antennae, Thorax, Corpus nigra, immaculata. Elytra teftacea apice rotundata nec emarginata. Pedes nigui.

2. CVCVIVS ruber, thorace fulcato, fe-clati: moribus clauatis rufis.

Habitat in America boreali Dom. Yeats.

Corpus depreffum. Antennae breues, niorae articuiis vndecim. Caput glabrum, rufum, poftice vtrinque rotundarum. Os palleicens. Thorax rufus, bifulcatus. Scutelium parum, rotundatum. Elytra rufa margine nigricante. Abdomen nigricans. Pedes nigi femoribus clauaris rufis.

3. CVCVIYS? thorace denticulato rufo, dubius clytris nigris, antennis filiformibus lon. gitudine corporis.

Habitat in America boreali.

Statura deprefia praecedentium at mihi haud rite examinatus ideoque de gencre hae. reo. Antennae filiformes, flexuofac artiP 5

culo 
culo vltimo acuto. Caput rufum. Thorax denticulatus rufus. Elytra ftriata, nigra, obtufa. Corpus cum pedibus rufum.

pulica- 3-4. MALACHIVS niger, thoracis marginibus elyrrorumque apicibus rubris.

Habitat Chilonii Dom. Lund.

Affinis M. pediculario, cuius forte mera varietas at thoracis latera rubra.

soesus 2-3. ELATER ater, thorace maculis duabus ocellaribus, elytris ftrigis duabus arcuatis punctoque poftico ferrugineis.

Habitat in Africa Dom. lee.

Corpus maiufculum. Caput laene, nitidum, atrum, immaculatum. Thorax ater vtrinque ocello coeco fiue annulo magno, oblongo, ferrugineo. Elytra lacuia, atra frigis duabus anteriore antrorfum, poferiore retrorfum arcuata. Poftice punctum oblongum obícurius.

fulca. 8-9. ELATER villofa-albidus, elytris ftriis tribus eleuatis glabris atris.

Habitat in America boreali Dom. Spengler.

Magnitudo \& fumma affinitas E. porcati, cuius forte iunior varietas. Caput atrum, obfcurum antennis breuibus ferratis. Tharax villofo albidus antice nigricans. Pecto. ris futura atra, glabra. Elytra villoforalbida ftriis tribus eleuatis, atris, nitidis. Ab. domen fubrus vti \& pedes villolo-albida. 
28-29. ELATER niger, thorace pube-baema fcente fuluo, elytris fanguineis. Elater antennis pectinaris fecundus Schaeff. Icon. I. tab. 2. fig. 6. potius hic pertinere viderur, quamuis tho. rax niger.

Habitat in Germania.

Paullo maior E. fanguineo. Antennae ferratae, nigrae. Thorax pubefcens, fuluus. Scutellum nigrum. Elytra fubftriata, fanguinea.

33-34. ELA TER thorace atro nitido, ely-limbatris teftaceis: limbo nigro. tus

Habitat Chilonii Dom. Lund.

Statura \& magnitudo E. minuti, cui valde affinis, at elytra reftacea margine omni nigro.

8-9. BVPRESTIS elytris tridentatis viri-ruti-

dibus nigro maculatis: margine aureo. lans

Habitat in Auftria Dr. Schulz.

Media. Caput virefcens antennis fufcis. Thorax fcaber viridi-nitens margine aureo. Elytra tridentata, itriata, viridia margine aureo punctis numerofis nigris. Corpus fubtus viride, nitens.

22 - 23. BVPRESTIS elytris integris, aurea Tro. nitidifima, dorío elytris pedibusque vi.cbilus ridibus.

Habitat in Autria Dr. Schulz. Statura \& magnitudo B.8 guttatae. Caput aureum 


\section{6

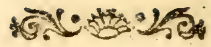

aureum antennis viridifufcis. Thorax anreus, nitidifimus dorfo coeruleo linea me. dia viridi. Elyrra integra, laeuia, viridia, nirida. Corpus aureum, nitidifimum. $\mathrm{Pe}$. des virides, nitidi.

plebeia 28-29. BVPRESTIS elytris emarginatis cupreis fufco maculatis.

Habitat in Indiis Dr. Schulz.

Corpus magnitudine \& ftatura B. ruficae. Antennae nigrae. Caput \& thorax obfcura, fcabra. Elytra emarginata, obfcure cuprea maculis plurimis fubconfuentitus fufcisa Corpus cupreum, nitidum. Abdomen fupra viride.

hugu. 28-29. BVPRESTIS elytris integris ob. Eris fcuris nigra fcabris, corpore fubrus cupreo.

Habitat in Auftria Dr. Schulz.

Corpus magnizudine \& farura B. Tenebrionis. Caput obfcurum, grifeum antennis rigris. Thorax obicurus, grifeus, lae. uis margine auro panco nitidulo. Elytra incegra, obfcura, grifea punctis plurimis, eleuatis, oblongis, glabris fcabra. Cor. pus fubtus cupreum, nitidum.

$4 m \sigma-3 \mathrm{I}-32$. BVPRESTIS elycris inteoris viriculata dis, thorace poltice elytrisqu: maculis duabus aureis.

Habitat in India Dr. Fothergill. Starura parua nofiratum. Thorax viridis pofti- 
poftice late aureus. Elytra integra viridia maculis ciuabus magnis aureis.

39-40. BVPRESTIS elytris integerrimis bigus.

linearibus viridibus puncto albo, abdo taik mine cyaneo punctis tribus albis.

Habirat in Anglia Dom. Yeats.

Paullo maior B. viridi. Caput viride fronre imprefla antennis brenibus nigris. Thorax cylindricus, viridis, immaculatus. Elytra linearia, viridia puncto paruo albo ad futuram. Abdomen fupra cyaneum, nitidum vtrinque punctis tribus albis, quorum anterius parum remotum. Subtus itidem coeruleum vtrinque punctis tribus albis.

$4 \mathrm{I}-42$. BVPRESTIS elytris in tegerrimis, nitidu thorace marginaio vtrinque depreffo, corpore viridi nitido. Linn. Sytt. Nat. 2. 662.15. Fn. Sv. 757 .

Habitat in Europa.

Corpus totum viride, nitidum. Elyrra paullo obfcuriora, attamen nitida. $4 \mathrm{I}-42$. BVPRESTIS elytris integerrimis Salicis viridis nitens, colcoptris aureis bafi viridibus.

Schaeff. Icnn. tab. 31. fig. I 2.

Habitat in Germaniae Salice Dr. Schulz.

Statura \& magnitudo B. nitidulae. Capur viride, pubefcens antennis fufcis. Thorax depreifus, marginatus, viriơis maculis duabus cyaneo-impretis. Elytra integra rufo- 
aurea bafi in primis ad futuram viridibus. Subtus viridis immaculata.

pructu-3-4. DYTISCVS niger, clypeo elytrobatus rumque margine exteriori albis, elytris ftriis tribus punctatis.

Dyticus fuicus margine coleoptrorum thoracisque flauo Geoff. Inf. I. 185. I.

Roef. Inf. 2. Aquatil. r. tab. 2.

Frifh. Inf. 13. tab. r. fig. 7.

Habitat Chilonii in riuulis fyluaticis Dom. Lund.

Magnitudo \& ftatura omnino D. marginalis. Caput atrum clypeo albo. Thorax ater nitidus margine folo laterali albo. Elytra nigra, nitida ftriis tribus punctaris exa. rata margineque exteriori albido, qui tamen color apicem elytrorum haud attingit. Corpus nigrum tibiis anticis pallefcentibus.

Faemina fulcis decem dimidiatis elytrorum vti in D. Cemiftriato.

gibbus 14-15. DYTISCVS gibbus ferrugineus, elytris nigris apice acuminatis. Habirat Chilonii in aquis ftagnantibus Dom. de Seheftedt.

Statura D. erythrocephali at paullo minor. Caput ferrugineum bafi parum nigricante. Thorax ferrugineus margine poftico nigro. Elytra laeuia, nigra bafi margineque obfolete ferrugineo maculata. Corpus \& pedes ferruginea abdomine apice nigro. 
Variat interdum capite thoraceque to. tis ferrugineis.

2I-22. DYTISCVS ater, capite ferrugi-6puftue neo, elyrris maculis tribus rufis: bafeos latus maiore.

Habitat in Sveciae aquis fagnantibus.

Minutus. Caput ferrugineum oculorum regione nigra. Thorax niger lateribus ferrugineis. Elytra glabra, lacuia, nigra maculis tribus ferrugineis prima bafeos maiore lunata, reliquis duabus verfus marginem. Corpus atrum pedibus ferrugineis.

25-26. DYTISCVS ferrugineus, elytris inae. nigris: lateribus inaequalicer ferrugineis. qualis Habitat in Sveciae aquis flagnantibus.

Magnitudo D. lineati. Caput ferrugineum oculorum orbita nigra. Thorax ferrugineus poftice niger. Elytra laeuia, nigra margine ferrugineo ramulos inaequales, abbreuiatos exferente. Subtus ferrugineus.

Variat interdum linea media elytrorum flaua a macula bafeos admedium marginis exterioris ducta.

2-3. CARABVS apterus niger violaceo cyaneus nirens, elytris punctis intricatis rugofis.

Habitat in Europa.

Minor C. coriario. Caput atrum antennis apice cinereis. Thorax obcordatus, planus, canaliculatus, violaceo nitens. Elytra haud

conna. 


\section{0}

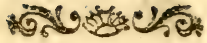

connata, attamen alas nullas continent. $E$. lytra punctis eleuatis, intricatis, plurimis rugera, nigra margine in primis violaceo, nitido. Corpus \& pedes atra.

limb-5I-52. CARABVS thorace elycrisque viths ricibus: margine fafcisque abbreuiatis pallidis.

Habirat Chilonii fub lapidum aceruis Dom. de Seheftedt.

Magnitudo C. melanocephali at latior. Antennae pallidae. Caput virefcens linea frontali antice bifida, albida. Os teftaceum maxillis apice nigris labioque albo. Thorax. laticudine fere elytrorum viridis margine antico \& laterali pallidis. Elytra viridia margine pallido, a quo fafciae duae fiexuofae abbreuiatae exeunt. Corpus teftaceum pedibus pallidis.

sitta. 56-57. CARABVS alatus, thorace mar. this

ginato rufo, elytris atris: vitta alba.

Habitat in America boreali. Yeats.

Statura C. crux minoris. Antennae nigrae. Caput rufum. Thorax rotundatus, marginatus, rufus. Scutellum paruum, rufum. Elytra glabra, atra vitta longitudinali alba. Margo rufécens. Corpus ferrugi. neum. Pedes nigri.

Morio 9-IO. PIMELIA thorace antice rotundato atra, antennis apice rufis.

Habitat in Anglia. 


\section{可沵次}

Antennae apice moniliformes articulo vltimo rufo. Caput atrum. Thorax antice rotundatus, poltice truncatus ater. Elytra ftriata. Corpus atrum.

4-5. HELOPS ater, thorace quadrato lae. Morio

ui, elytris punctato ftriaris.

Habirat in America boreali.

Affinis H. nigritae at paullo maior. Anrennae apice moniliformes articulo vltimo longiôri, ouato. Thorax laeuis, quadratus marginibus haud rorundatis, obfcurus. Elyrra punctato ftriata, obfcura. Corpus fub. tus \& pedes atra, nitida.

4-5. HELOPS ater, thoracis marginibus Nigri-

rotundatis, elytris crenato fulcatis.

Tencovio atratus oblongus ater, elytris fulcatis acutiufculis Syft. Ent. 2;6. 4.

Habitat in America meridionali.

Antennae apice moniliformes. Thorax laeuis margine exteriore rotundato anguioque poftico acuto. Plantae fubtus lana rufa. II - I 2. STAPHYLINVS niger, elytris 2 gut puncto albido, oculis prominulis Linn.tutus Sylt. Nar. 2. 685. 15. Fr. Sv. 85 r.

Habitat ad littora maris Balthici de Seheftedt.

Affinis S. bipuntulato, at differt manifefte thorace rotundiore \& oculis magis promiaulis. 


\section{2}

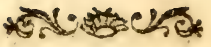

clani- I -12 . STAPHYLINVS niger immactilacornis rus, thorace rotundato laeui, antennis extrorium crafioribus.

\section{Habitat Kilonii Lund.}

Sratura \& magnirudo omnino S. 2 guttati. Antennae moniliformes, extrorfum crafliores. Thorax rotundatus, ater, nitens, laeuis. Elytra vix punctata, atra, immaculata. Abdomen \& pedes arra.

shore. I-2. OXYPORVS nigricans, thorace ru. siews fo, elytris fufcis bafi albis.

Habitat in Boleris. Captus Kilonii d. I I. Iul. de Seheftedt.

Statura praecedentium at duplo minor. Anrennae moniliformes, extrorfum craflio. res bafi pallidae apice nigrae. Caput atrum, nitidum. Thorax rufus, nitidus. Elytra fulca, bafi albida margine tamen crafliori fufco. Abdominis fegmenta picea vel po. tius bafi nigra apice rufefcentia. Pedes fla. uefcentes.

ryg. 2-3. OXYPORVS nigricans, thorace elymaens trisque pallidis.

Habitar in Boleris Captus Kilonii d. Ir. Iul. de Seheftedt.

Affinis praecedentiat minor. Caput atrum, nitidum. Thorax obficure pallidus. Elytra pallida margine cralfiori, fufco. Abdomen magis nigrum. 
I - 2. PAEDERVS niger, thorace rufo, ely ruficol. tris cyaneis.

Habitat Kilonii frequens fub lapidibus littoralibus de Seheftedt.

Minor P. ripario. Caput arrum, nitidum antennis moniliformibus nigris. Thorax gibbus, rufus, niridus. Elytra abbreuiata, cyanea, nitida. Abdomen \& pedes atra. 22. MANTIS thorace cylindrico flaueficen - pufilla te, elytris alisque hyalinis immaculatis. Mantis pufilla Pallas fpecill.Zool. fafic. IX. pag. I 5. tab. I. fig. 9 .

Habitai in Africa.

Vix Raphidia Ophiopfi maior.

$22-23$. GRYLLVS thorace fubcarinato, fibisiantennis clauatis, tibiis anticis ouato cla-cus uatis † Linn. Syft. Nat. 2. 701. 5 I.

Gryllus cluuimanus Pall. Spic. Zool. farc. IX. p. 2 I. tab. r. fig. I I.

Habitat in Sibiria.

7-8. ONISCVS cauda quadridentata. linea. Linn. Syft. Nat. I I. 1060. 9. Amoen. acad. ris 6. 415.100 .

Onifcus vngulatus Pall. fpic. Zool. farc. IX. pag. 62. tab. 4. fig. I I.

Habitat in Occano Indico.

87-18. ONISCVS oualis, cauda obtufa, asfimi. mutica Linn. Syft. Nat. 2. 106 I. 13.

Onifcus globator Pall. fpicill. Zool. fafc. IX. pag. 70. tab. 4 . fig. 18 ?

Buft. 
Baft. Subfe. p. 144. tab. 13. fig. 3 .

Habitat in Oceano Europaeo.

fignata I. PODVRA fubglobofa fufca, abdominis lateribus fuluo maculatis.

Podria fufco nigra, abdomine globofo fignaturis ferrugineis Geoff. Inf. 3 . 607. I.

Habitat in locis humentibus.

Antennae longitudine fere corporis.

halte. 4-5. EPHEMERA cauda trifeta fufca, ab. rata domine albido apice fufco.

Habitat Kilonis frequens.

Parus. Caput \& thorax fufca, immaculata. Abdomen album apice fufcum. Cauda fetis tribus corpore triplo longioribus. Alae duae magnae, albae margine crafíiori nigricante. Pedes antici porrecti, albi.

grifens I-2. HEMEROBIVS alis grifeis alboma chilatis.

Habitat in Africa Dom. Yeats.

Antennae defunt. Statura H. peetinicoro nis at minor. Caput \& thorax ferruginea nigro-varia. Alae magnae, grifeae itriga punctara media punctisque fparfis apicis albis: Alae polticae bafi nigrae.

mitisu-5-6. HEMEROBIVS alis fufco nitidulis hus corpore teftaceo, pedibus pallidis. Habitat in Germania de Hattorf. Magnitudo H. hirti. Antennac longitu. 
dine corporis fufcae. Caput teftacelim oculis magnis nigris. Thorax \& abdomen teftacea, immaculata. Alae corpore multo maiores, fufcae auro rubroque nitidifimae, immaculatae. Pedes pallidi.

7-8. HEMEROBIVS niger ore pedibus. longique pallidis, antennis longioribus fufcis. comis Habitat Kilonii in praedio nobilifím: comitis Holk Ekhoffdisto.

Paullo H. hirto minor. Antennae corpore triplo longiores fufcae, immaculatae. Caput nigrum ore pallido. Thorax \& abdomen nigra, immaculata. Alac albae neruis punctoque marginali fufcis. Pedes pallidi.

6. PANORPA alis anticis flawo fufcoque halte. variegatis; pofticis longilfimis fufcis : ma- ruia culis tribus albis.

Panorpa buittrut. Fordk. difcript. anim. 97. tab. 25. fig. E.

Habitat in Oriente.

Statura \& fumma affinitas P. Coac. An. rennae filiformes, nigrae. Corpus nigrum. Alae anticàe magnae, crectae, flawo fufcoque variegatas. Pofticae longitiumse, lineares, apice parum latiores, nigrae maculis tribus albis tertia alam terminante.

5I-52. ICHNEVMON ater, abdomine polvce lineari longiffimo, tibiis pofticis clauatis. ${ }^{* a t o v}$ Habitat in India.

Q3 Magni- 
Magnitudo T. manifeftatoris. Corpus to. tum atrum, nitidum. Abdomen longiflmum, lineare fegmentis cylindricis. Alae breues, albae. Pedes atri tibiis pofticis clauatis.

reca- 74-75. ICHNEVMON niger, abdomine tor

flauo apice nigro, pedibus flauis.

Roef. Inf. 2. Vefp. IV. tab. 4 .

Habirat in Phalaenarum laruis, quas folliculis affociatis albis obtegुunt.

Minutus. Antennae feraceae, nigrae. Thorax niger, nitidus, immaculatus. Abdomen flaum apice nigrum. Pedes flaui. Alae hyalinae macula coltali nigra.

mari-3-4. ANDRENA thorace pubefcente, abwata domine ferrugineo: fegmentorum mar. ginibus cinereis; primo toto atro.

Habitat in Germania de Hattorf.

Parua ftatura A. heluolae. Capur \& thorax nigra lana cinerea pubefcentia. Abdo. minis primum fegmentum atrum reliqua fer. ruginea marginibus cinereis.

foroe. 8-9. APIS hirfuta atra, ano albo.

Habitat in Daniae nemoribus. Dom. Schlanbufch.

Statura omnino reliquorum at tota atra folo ano albo.

Arbu- I 3 - r 4. APIS hirfuta nigra, thorace antice flauo, ano rubro.

Habi- 


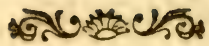

Habitat in Daniae fyluis Dom. Schlanbufch.

Statura \& magnitudo A. hortorum. Caput nigrum fronte pilis flauis. Thorax hirtus, niger antice fafcia flaua \& poftice pilis rariffimis fauis. Abdomen hirtum nigrum ano tate rufo.

22--3. APIS hirfuta atra, fcurello abdo-mnio. mineque pallefcentibus, ano rufo.

Habitat in Chilonii nemoribus Dom. de Seheftedit.

Praecedentibus valde affinis at paullo minor. Caput \& thorax hirta, atra, imma. culata. Scutelli regio vti \& abdomen pilis pallidis recta. Anus rufefcens. Pedes nigri.

54-55. APIS nigra, abdomine cylindri- 4 cis.

co; fafciis quartuor albis, pedibus hauis. Eza Habitat in Daniae nemoribus Schlan. bufch.

Magnitudo A. florifomnis. Antennae fupra fulcae, fubtus fuluae. Corpus nigrum pube parua cinerafcente. Abdomen cylindricum fegmentorum q̣uatuor marginibus albis anteriorum interruptis. Pedes flaui.

3. TERMES thorace dentato. $t$

Termes arda Forfkahl defcript. anim. pag. 96. n. 6r. tab. 25. fig. A.

Habitat in Aegypto fornices cylindri. cos progrediendo inaedificans. De.

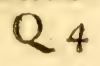

struens 
ftruens cibaria, arbores, fructus omniaque. Praecedenti valde affinis an diftincta.

Cylin. 33-34. CANCER thorace bifulcato: latedrus ribus dilatato cylindricis apice fpinolis.

Habitat Tranquebariae.

Statura fingularis.

Frons obtufa, canaliculata. Thorax globofus fulcis duobus magnis vnicoque antico transuerfali impreflis intus hirtis. Latera thoracis vltra longitudinem corporis dilata. ta, cylindrica, albida punctis rubris fcabra apiceque fpina alba, valida armata. Cauda fub abdomine inflexa, albida, fcabra in medòic longirudinaliter bifulcata. Pedes mihi defunt.

grofja. I. GAMMARVS manibus adactylis lonper gitudine corporis.

Cancer grofipse Linn. Sylt. Nat. 2.1055.80. Afacus muticus pede antico fubulato edentulo longifimo craffifimo Gronov. Zooph. 989. tab. 17. fig. 7 .

Onifcus volutator Pallas Spic. Zool, fafcic. IX. p. 59. tab. 4. fig 9 .

Habitat in Europae aquis ftagnantibus. nobiti- 3. AGRION alis ereetis: pofticis fupraviridi-tericeis apice atris.

Habitat in America meridionaliSpengler. Statura \& magnitudo A. Virgo. Corpus totum viride, nitidum. Alae anticae reticulatae, 


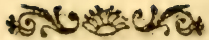

eulatse, obfcurae, pofticae viridi fericeae, nitidae apice \& fubtus atrae.

4. AGRION alis reticulatis, abdomine lon-lines. gillimo lineari.

Habitat in India Dr. Fothergill.

Statura A. puellae at maior. Corpus fu. fourn abdomine lineari, longiffimo. Alae porrectae, reticulatae, anticis macula apicis oblonga, alba, pofticis fufca.

3-4. ARANEA virefcens, thoracis lateri-fignato

bus abdominisque lineis duabus nigris.

Habitat in nemoribus Kilonienfibus capt. d. XI. Iul.

Parua. Caput virefcens atomis aliquot nigris. Thorax itidem virefcens lareribus fipperne-late nigris. Abdomen globofum, virefens vtrinque linea lata e maculis quaruor transuerfis nigris, quae tamen lineac nec bafin nec apicem abdominis attingunt. Papilli textorii prominent. Pedes virides puncis nigris.

8-9. ARANEA viridis, abdominis dorfo dorfos
fufco.

Habitat in nemoribus Kilonienfibus.

Parua. Caput, thorax \& pedes viridia, immaculata. Abdomen ouatum, fubtus palliduris fipra fufcum limbo pallefcente.

33-34. ARANEA cinerea, abdominis ciseres dorfo fufco: punctis octo cinereis.

Q 5

Habi- 
Habitat ad littora finus Kilonienfis.

Media. Maxillae cinereae vnguibus nigris. Oculi quatuor inferiores minutiffimi quatuor fuperiores multo maiores. Thorax cinereus, immaculatus. Abdomen ouarum dorfo obfcuro punctis quatuor parium cinereis, minutiffinis. Pedes cinerei fufco an. nulati femoribus immaculatis.

yopa $35-36$. ARANEA virefcens, abdominis dorfo late fanguineo.

Habitat in praedio nobiliffimi comitis Holk Ekhoff diato.

Caput \& thorax pallide virefcentia maxillis apice nigris lineisque duabus dorfalibus thoracis obficurioribus. Abdomen ouatum, pubefcens fupra dorfo fang uineo punctis aliquot nigris, lateribus flauefcentibus, immaculatis. Papillae textoriae apice nigrae. Pedes elongati, virefcentes tibiis anticis apice nigris.

Renus $16-17$. PAPILIO, E. T. alis dentatis fubconcoloribus nigris: pofticis vtrinque maculis flauis marginalibus.

Papilio Hippolytlbus Cram. Inf. 1. 13. tab. io. fig. A. B.

Seba Mul. 4. tab. 46. fig. Ir. I2.

Habitat in Amboina.

Magnus Alae pofticae fubrus paullo albidiores. Margo alarum pofticarum cingitur vtrinque maculis fex inaequalibus flatis. 
22-23. PAPILIO. E. T. alis dentatis nigro Andio. aeneis: anticis macula quadrifida flaua, geus pofticis fubtus lunulis rubris, cyaneis flauisque.

Papilio Androgeus Cram. Inf. 2. tab. I6. fig. C. $D$.

Habitat Surinami.

Affinis P. Polydamas.

23-24. PAPILIO. E. T. alis dentatis atris: Ly faw. anticis ftriga alba, pofticis macula media der alba lunulisque rubris.

Habitat in India Dr. Fothergill.

Magnitudo \& Atatura P. Aeneas. Alae anticae atrae ftriga media alba. Pofticae dentatae, atrae macula media magna alba pofticeque lunulis tribus anguloque ani rubris.

30-3r. PAPILIO. E. A. alis dentato bicau cafor dacis fuluis margine nigris: fubtus fafcia maculisque albis nigra foetis.

Papilio Caftor Cram. Inf.4.tab.37.fig.E.F.

Habitat in Guinea.

Starura praecedentium. Alae fubtus purpurafcentes bafi maculis variis albis nigra foetis, in medio faficia alba apice maculis oceilaribus.

Conf. Pupilie Pelias Cram. Inf. 1. tab. 3. fig. C. D. vix diftincta fpecies viderur. 30-3r. PAPILIO. E. A. alis dentato-bi-Pollux caudatus fufcis fafcia flaua: futtus fafcia maculisque aibis nigra foetis.

Pa. 
Papilio Pollux Cram. Inf. 4. tab. 37. fig. C. D.

Habitat in Guinea.

Nimis praecedenti affinis. Differt tamen colore fupra fufco punctisque marginalibus coeruleis \& fubrus defectu macularum ocel. latarum.

Cre. 40-4r. PAPILFO. E. A. alis caudatis: futhors pra fufcis, fubtus glaucis, fafcia vtrinque alba.

Habitat Surinami Dr. Schulz.

Paruus. Antennae extrorfum cralfiores haud capiratae. Corpus fufcum. Alae anticae fufcae bafi frigis aliquot obfcurioribus, in medio fafcia lata alba, apice nigrae punatis fex aut feptem albis. Subrus glaucae fa. fcia alba ftrigisque fuluis. Pofticae fufcae ftriga continuata alba, quae tamen angulum ani haud attingit. Margo pofticus ftrigofus maculis tribus acellaribus arris iride alba. Ad angalum ani macula parua rufa. Cauda elongata atra margine albo. Subtus glaucae fafcia lata alba ftrigisque fuluis poftice punfitis tribus atris punctoque ani rufo.

Oyfilo $40-4 \mathrm{r}$. PAPILIO. E. A. alis caudatis fufcis: cloits

anticis fafciis duabus, pofticis vnica albis, fubrus bafi albis fafcia filua.

Habirat Surinami Dr. Schulz.

Affinis praecedenti eiusdemque ftaturae \& magnitudinis. Alae anticae fufcae fafciis duabus 
duabus albis pofferiore lineari, fubtus bati albae fafcia fulua, apice fufcae frigis duabus glaucis, exteriore extrorfum bifida. Pofticae fufcae fafcia continuata alba, quae angulum ani haud attingit. Maculae duae atrae iride alba adangulum ani. Angulus ani appendiculatus ferrugineus. Cauda elongata, atra margine albo. Subrus bafi albae fafcia fulua, apice fufcae itriga glauca. Margo nigrro-ftrigofus. Cauda nigra margine albo. Angulus ani macula rufa puncto atro.

60-6r. PAPILIO. E. A. alis dentatis nigris Amphio fafcia inaequali flana: pofticis fubtus ftri-trion ga e punctis fiauis lunulisque cyaneis.

Pupilio Amphitrion Cram. Inf. 1. tab. 7。 fig. A. B.

Seb. Muf. 4. tab. 8. fig. 7.8.

Habitat in America.

Magnus. Corpus nigrum, immaculatum. Alae anticae concolores, nigrae maculis quaruo: onatis fafciam fere conftituentibus inrerruptam. Pofticae nigrae fafcia inaequali verfus angulum ani attenuata flaua, quae tamen margines haud attingit. Subrus nigrae Atriga e punctis feptem magnis orbiculatis flauis \& alia ftriga e lunulis feptem coeruleis, quae ad angulum ani puncto flauo termina. tur. Margo ipfe finubus flauis. 66-67. PAPILIO E. A. alis angulato-re-Auropandis fufcis: fubrus liuidiufculis ocello medom anguli ani. 


\section{मख.}

Papilio Automedon Cram. Inf. 4. tab. 4 r. fig. A. B.

Habitat Surinami.

Magnitudo \& Atatura P. Tencer. Alae maris coeruleo colore nitidae, foeminae obfcuriores. Subtus lucidae ftriga obliqua bafeos alba verfusque angulum ani ocello coe. rulefcente.

Hecale $80-8 \mathrm{r}$. PAPILIO $\mathrm{H}$. alis oblongis integerrimis nigris: anticis falcia pofticis fubrus punctis marginalibus albis.

Papilio Pajuthoc Crams. Inf. 2. tab. I7. fig. A. B.

Habitat Surinami.

Magnus. Caput atrum flauo punçatum. Corpus nigrum abdomine fubtus flauo. A. lae anticae nigrae falcia lata alba, in qua: puncta aliquor nigra, poftice bifida. Puncta alba verfus apicem, fubtus concolores. Pofticae fupra atrae, immaculatae, fubtus nigrae bafi parum flauefientes \& apice ftriga punctorum albidorum.

Bra00-8I --82. PAPILIC H. alis oblongis integris lis

fufcis : anticis flauo macularis, polticis fubtus fanguineo radiatis.

Papilio Bellona Cram. Inf. 2. tab. 13. fig. E. F.

Habitat Surinami.

Statura P. Myrti. Alae anticae vtrinque flauo maculatae, polticae fupra immaculatae, fubtus rubro radiatae.

$81-82$. 
8r - 82. PAPILIO. H. alis oblongis integris Tbalkt fubconcoloribus atris bafi rubro radiatis: anticis flauo macularis.

Papilio Thales Cram. Inf. 4. tab. 38. fig. C D. Aub. Mifcell. tab. 72. fig. 3. 4 .

Habitat Surinami.

Praecedentibus affinis. Alae omnes bafi rufo radiatae, antic $x$ flauo vtrinque maculatae. 83-84. PAPILIO. H. alis oblongis integris Aegle atris: anticis maculis, polticis difco hyalinis.

Habitat in India Dr. Schulz.

Statura omnino P. Pfidii at triplo minor. Alae anticae fupra atrae macula magna cuneiformi bafeos fafcia atra diuifa. Verfus apicem maculae duae maiores \& tres minores hyalinae. Subtus concolores at inter maculas litura fulua \& puncta tria apicis alba. Pofticae fupra arrae difio hyalino fafciaque fubmarginali abbreuiata fulua. Subtus difco hyalino, qui includitur linea atra, tunc maiori fulua margine tamen ipfo atro punctis tribus apicis albis.

I26-27. PAPILIO. D. C. alis rotundatis Edus integerrimis albis fufco macularis: pofticis fubtus virefcentibus albo maculatis.

Habitat Chilonii Dom. de Seheftedt. Statura \& magnitudo P. Cardamines. Antennae fufcae claua apice alba. Alae anticae albae in medio maculis duabus nigris altera 


\section{6}

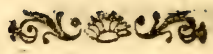

ad marginem craffiorem, altera ad renuio. rem; apice nigrae albo punctatae. Pofti. cae fupra albae margine nigro maculato. Subtus anticae concolores at maculis magis virefcentibus, pofticae late virides maculis tribus bafeos, fafcia media admarginem tenuiorem interrupta maculisque marginalibus albis.

Leucip. $\mathrm{I}_{3} 2-33$. PAPILIO. D. C. alis integerrimis pe fuluis: pofticis fupra flauis.

Papilio Leucippe Cram. Inl. 3. tab. $3^{6 .}$ fig. A. B. C.

Habitat in India orientali, Amboina.

Magnus. Alae anticae fupra fuluae punctis marginalibus atris, fubtus fuluae imma. culatae. Pofticae fupra flauiffimae (foeminae) maculis marginalibus nigris, fubtus pallide fuluae, atomis nigris.

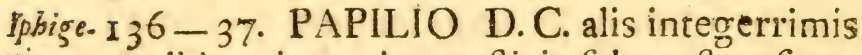
nia albis apice atris: pofticis fubtus flauefcentibus fafcia media atra; ftriga abbreuiata fulua.

Habitat Surinami Dr. Schulz.

Medius. Alae omnes fupra albae anticis apice taris. Margo poiticus nigrefcit. Subtus alae anticae concolores, pofticae flauefcentes ftriga bafeos atra. In medio fafcia lata abbreuiata atra, in qua ftriga etiam abbreuiata fulua. In margine ipfo maculae nigrae, quarum anteriores maiores.

$\pi 44-45$ 
144-45. PAPILIO D. C. alis rotundatis fla. Ss ra

uis apice (medio fuluo) atris: pofticis fub. tus maculis feptem ocellaribus albis.

Habitat in America Dom. Spengler.

Medius. Antennae ciauatae, nigrae claua apice ferruginea. Alae anricae flauilimae apice nigrac macula media fulua. Pofticae rorundarae, flatirimae margine nigro. Sub. tus omnes flaullimae punctis marginal bus atris \& in pofticis puncta feptem alba iride ferruginea cincta.

160-61. PAPILIO D. F. alis integerrimis cypa nigris: fafciis duabus albis; anteriorum riffias obliģuis, pofticarum punctatis. Papilio Dacdalus Cram. Inf. ı. tab. I. fig.A.B. Habirat in America meridionali.

Antennae clauatae claua acuminata. Alae nigrae certo fitu viridi colore fplendentes. I67-68. PAIILIO D. F. alis integerrimis Galas.

fupra atris fanguineo - fafciatis : anticis tbis punctis duobus apicis albis.

Papilio Galantbis Cramer Inf. 3. tab. 25. fig: D. E.

Habitat Surinami.

Medius. Alae anticae atrae bafi fafciaque ranguineis. Puncta ciuo parua alba approximata oblonga apicis. Pofticae atrae fafcia obliqua fanguinea. Subtus anticae flauae fuluo irroratae fafciis duabus oliuaceis pofteriore apicis. Pofticae bafi fulcae apice flauefcentes fafcia oliuacea.

R

$1 \div 2 \cdot 73$. 
Gilip. 172-73. PAPIIIO D. F. alis intererrimis pus

concolonibus fuluis aliso maculatis: margine atro punctis albis.

Pupilio Gilippus Cram.1n?.3.tab.26. fig.C.D. Habirat in America nerrdionali.

Statura ormino P. Alíppi at paullo minor. Aife omnes fulune albo maculatae. Margo pofticus arer frigis tribus puncto. rum alborum.

Lacin- 184-85. PAPILIO D. F. alis integerrimis $d a$

nigro fafciatis: fupra coeruleis, fubtus fuluis.

Pupilio Lucinda Cram. Inf. r. tab. r. fig. E. F. Seb. Muf. 4. tab. I3. fig. $25 \cdot 26$.

Habitat Surinami.

Paruus ftacura P. Morpheus. Striga punctorum nigrorum verius marginem in anticis fubrus, in pofticis vtringue.

lairus $187-88$. PAPILIO D. F. alis integerrimis fufcis: pofticis fupra ocello fubrus duo. bus; poltico fesquialtero.

Papilio lairus Cram. Inf. I. p. 9. tab. 6. fig. A. B.

Habitat in India orientali.

Magnus. Alae omnes integerrimae, fufcae anticis falcia, pofticis bafi albidis. $\mathrm{O}$ cellus magnus coeruleus iride fiaua pupillaque alba alarum pofticarum \& fubtus duo eiusdem coloris, quorum pofteriori ocellus minor itidem pupillatus adnatus.

$189 \cdot 90$. 
189-90. PAPILIO D. F. alis integerrimis Pbilo. fufcis: fubrus anticis ocello, pofticis li-cles neis transuerfis vadatis. Limu. Syft. Nat. 2. 791 . 240. Muf. Lud. Vlr. 32 r. Clerk. Icon. tab. 45 . fig. 3 .

Habitat in Indiis.

Variat alis fupra atris fafcia coerulefcente maculaque mayna alba ani \& alis fuccis lineis transuerfis vindatis fafciague alba.

189-90. PAPILIO D. F. alis integerrimis Croefus cyaneis ftrigis atris numerofis: anticis fupra macula ocellari tripupillata.

Habitat Surinami Dr. Schulz.

Alae anticae fupra cyaneae ftrigis aliquot apiceque atris. In medio macula magna atra punctis tribus paruis albis. Snbtus cyaneae, nitidae arcu apiceque atris. Pofticae vtrinque cyaneae ftrigis numerofis vndaris atris. 20r - 2. PAPILIO D. F. alis integerrimis Daums

fuluis: anticis ocellis duobus, pofticis fex coecis, fubtus pupillatis.

Habitat Hamburgi Dr. Schulz, Kilonii Seheftedt.

Medius. Alae anticae fupra fuluae ocellis ducbus atris coecis tertioque minutiffimo vix diftincto; fubtus fafcia alba ocellis duobus pupilla alba. Pofticae obfcuriores ocellis yuinque aut fex coecis; fubtus grifeae fafcia interrupta alba ocellis fex atris pupilla alba pofteriore didymo.

$$
R_{2} 216-1 \%
$$


Ocype 216-I7. PAPIL.1O N. G. alis dentatis fixto pra fufcis immaculatis fubtus glancis ftrigistribus obfcuris: pofticis ocellis quinque. Habitat Surinami Di. Schuiz.

Paullo maior P. Penelope. Alae fupra omres fufiae, immaculatae friga marginali flauefcente. Subtus omnes glaucae, anticis frigis tribus obfcuris, pofteriore vndata, polticis thrigis quatnor bafeos fere obfoletis apicis vndata. Ocelli duo obroleti, minuti in apice alae anticae \& quinque maiores atri iride flaua pupilla alba in polticis inter ftri. gas vlrimas. Ocellus 2. 3. obfoleti.

Ocir. 216-17. PAPILIO N. G. alis dentatis fubes

pra abis apice fufcis, fubrus fufcis: fafciis duabus albis, pofticis ocellis quinque.

Habitat, Surinami Dr. Schulz.

Statura praecedentis. Alae anticae fupra albae apice fufcae, fubrus fufcae fafciis dua. bus albis. Ante marginem pofticum ocelli quatuor parui pupilla alba. Margo ipfe glau. cus ftrigis duabus obfcumoribus: anteriore vndata. Polticae fupra albae margine ftrigis duabus vndatis punctoque ante marginem nigris. Subtus fufcae fafciis duabus latis albis. Ocelli quinque ante marginem r. 4. maioribus atris, 5 minore atro, 2, 3 minoribus fufcis pupilla duplici argentea. Margo frigofus.

Amase 217-18. PAPLLIO. N. G. alis fubdentatis fufcis: 
fufcis: pofticis coerulefcentibus; fubtus oceliis quinque.

Habitat Surinami Dr. Schulz.

Statura praecedentis. Alae anticae fupra fufcae, immaculatae, fubtus frrigis duabus óbliquis obfcurioribus. Verfus a picem ocelli tres exteriori pupilla duplici, interioribus obfoletis. Pofticae fupra cyaneae, nitidae; fubrus cyaneae ftrigis duabus oblicuis fufcis. Ante marginem ocelli quinque 1. 4. maioribus atris, reliquis fufcis. Margo ftrigofus. 226-27. PAPILIO N. G. alis dentatis fu-perifcis: anticis ocello obfoleto; fubtus ftri-boca ga flauefiente, poftiǵs punctis ocellaribus atris.

Habitat Surinami Dr. Schutz.

Medius. Alae anticae fufcae ocelio ma* gno atro iride flanefiente pupilla alba, at obfoleta ad marginem anticum. Subsus grifeae ftrigga flauefcence punctis cluobus minueifi. mis albis. Pofticae fufcae ocello paruo obfoleto margineque ftrigofo. Subtus grifeae Atriga flauefcenre punctisque 5-6 paruis ocellaribus atris.

$230-3$ r. PAPILFO N. G. alis dentatis fur Hefio

fcis fubtus ftriga obliqua alba: anticis o- $\boldsymbol{n z}$ cellis duobus pofticis fefqui rertiis.

Papilio Hefonc Cram. Inf. I. tab. I I. fig. C.D.

Habitat Surinami.

Alae fupra immaculatae. Subrus fafcia

$$
\mathrm{R}_{3}
$$

alba 
alba margines haud attingit. Ocelli furci pupilla alba iride fulua annulo nigro coeruleoque cincta.

Doris 232-33. PAPILIO N. G. alis dentatis fufcis: fubtus coeruleis nigro farciatis ocellis trium parium.

Papilio Doris Cram. Inf. I. tab. 8. fig. B.C. Habitat Surinami.

Starura praecedentium parua. Alae omnes fupra fuccae margine poftico alarum poficarum parum abicante. Subtus omnes coeruleae, nitidae fafcis quatuor nigris. In fafcia tertia ocelli fex per paria approximati nigri iride fulua pupillaque albida. Primum par ad maryinem exreriorem alae anticae, fecundum ad marginem exteriorem alae po. fîcae, \& tertium denique ad angulum ani.

Hiera $236-37$. P.PIL.O. N. G. alis dentatis fufcis: anticis vringue ocello féqui altero, pofticis fubrus netulofis ocellis fex. Schaeff. lcon tab. 58. fig. 2. 3 .

Hali $x$ in Furopae pratis fyluaricis.

Affuis P. Jurtina attamen diftindta videtur. Alae anticac fufiae vtringue litura fiaua. $\mathrm{O}$. celíus magnus ater pupilla alba in altero fexu duplicata. Inter hunc \& apicem ocellus minutilimus pupilla alba. Ṕofticae fupra fufcae ncellis tribus pupilla alba exteriore coero; fibus fulco inereoque nebulofae ocellis fex incriore duplicato. 
$236-37$. PAPILIO N. G. alis dentatis fu. Tif. fcis: antivis ocellis vtrinque duobus po phome ficis fex: pupitia violacea.

Papulio Tifiplone Naturforfch. C. 18. tab. I. fig. $x-2$.

Habirat in Germaniae fyluis.

Menitudo \& fatura praecedentis. Alae anticae fufcae fafcia abbreniara flawa. Verfus apicem ocelli duo nigri iride flatua pupillaque violacea. Pofticae fufcae verfus marginem potictim ocelli fex nigri iride flaua pupillaque violacea fubtus omnes concolores.

$242-43$. PAPILIO N. G. alis crenatis ni- Clatb gris area languinea: ponticis fubrus mar- dia moratis ocellis feprem.

Habitat in India Dr. Schulz.

Magnitudo P. Tubrghise. Palpi albi apice nigri. Corpus fufum. Alae fupra omnes nigrae area, lata laete fanguines. Subtus anticae rubrae macula alba tribusque marginalibus atris, apice nigrae friga maculisque duabus obfoletis, marginalibus, cinercis. Pofticae bafi nigro cinereoglue marmoratae. In medio ftriga fexuofa firua coerulefensque, apice teftaceae nceliis feptem atris pupilla cocrulea: interiore duplicata. Striga marginalis atra.

$247-48$. PAPILIO N. G. alis dentatis atris: Calipso

pofticis fafcia fanguinea ocellis fupra quin. que fubtus feptem. 
Papilio Califto Cram. InC. 2. tab. 24. fig. A.B. D'Aubenton mifcell. tab. 20. fig. 2. 3 . Seba Muf. 4. tab. 44. fig. I2. 13. Habitat in Africa.

Magnus. Ala poftica fupra atra fafcia lata antice alba poftice rubra ocellis quinque atris pupilla coeruleícente, fubrus fufcæ fafcia rubra ocellis feptem vltimo didymo.

250-5I. PAPILIO N. P. alis bicandatadentatis fuluis ftrigis nigris: pofticis fubtus glaucis nigro punctatis.

Habitar in America meridionali D. Spengler.

Magnirudo P. Troglodyta. Antenna clauatæ fupra nigræ, fubtus albæ. Corpus fupra ferrugineo-hirtum, fubtus albidum. Alye anticæ falcatæ, dentatæ, fuluxe frigis tribus margineque exteriori nigris. Ante Itrigam priorem lineola transuerla puncta. que quatuor parua nigra. Inter ftrigam $\mathbf{~} .2$. lineola transuerfa \& adangulum ani litura nigra. Subtus fufco glaucoque variegatx. Poftica dentatæ, bicaudatre cauda inceriori breuiori, fuluæ frigis quatuor nigris. Adangulum ani puncta duo nigra annulo albo cincta lunulæque duæe albæe inter caudas. Subtus glaucre, bafi obfcuriores friga obliqua furca, verfus marginem pofticum ftriga punctorum airorum fubocellatorum.

Progme 264-65. PAPILIO. N. P. alis caudaro den- 
tatis fuluis punctis margineque albo punctato nigris: fibtus grileis.

$$
\begin{aligned}
& \text { Papilio Progm Cram. Int. 1.tal. 5.f.g.E.F. } \\
& \text { Hiabtat in America mericlionali. }
\end{aligned}
$$

Simillimus P. C. albo, at margo pofticus ahoum niger in pofticis albo punctarus.

271-72. PAPILIO. N. P. alis dentaris fla-Fyppr.

uis nigro variegaris apice ratiaris: polti-pyle

cis punctis leprem rubris.

Papilo Fiypermeftra Scop. Ent. 425.

Roel Inf. 4. tab. $\%$

Catesb. Carol. 2. 95 . tab. 95 .

Habitat in Europa autraliori, America.

280 - 8r. PAPILIO N. P. alis dentato cau- Hippo.

datis flawo nigroque varis: pofticis plin-na

ctis quatuor albis.

Habitat in India Dr. Schulz.

Magnus. Alice anticie dentatce apice interiori truncate \& fere emarninate fupra bafin fulux, tunc nigræe fafcia lara maculis duabus apicis flauis, fubtus variegarce. Poftica apice truncati, dentatie cauda elongata baif fuluce apice atra puncis quatuor albis. Subtus glauco fuficoque variegarie. 299-300. PAPILIO N. P. alis dentatis a!- Herrs-

bis: limbo fuluo, pofticis fubius fuluis, lia fariis duabus pundogue medio albis.

Habitat in India Dr. Schulz.

Paruus. Ale antice fupra alba bafi fub. fufcefcentes apice fulure maroine dentato

$$
\text { R } 5 \text { nigro. }
$$




\section{6}

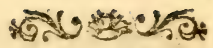

nigro. Subtus teftaceae macula magna difci punctisque aliquot verfus apicem albis. Po. fticae fupra albre limbo fuluo nigro marginato. Subtus fulune fafcia bafeos alba integra, tunc punctum medium album, tum fa. icia e maculis feptem albis, demum maculae feptern albae obfoletae marginales. Margo ipfe renuilfime niger.

Fy 30 3 $304-5$. PAPILIO N P. alis dentatis fuluis nigro punctatis: pofticis bali fupra fulcis, fubtus albidis.

Papilio Hyllus Cram. Inf.4 tab. 43 . E. B.C. Habitat in Oriente.

Minor P. Cinxiae. Alae anticae vtrinque fuluae nigro-punctatae fupra margine fufco. Polticae nigro punctarae fupra bafi fufcae apice fuluze, fubrus bafi albidae apice fuluae.

(jama 308 -9. PAPILIO N. P. alin dentatis fufcis atro flauoque variis: ponticis fubrus viridibus maculis tribus fafciisque duabus argenteis.

Papilio Maj a Cram. InC. 3. tab.25. fig. B.C. Habitat in Oriente.

Statura 12. Paphia. Alae omnes fupra fufae margine fhuo atro maculato. Subrus anticae fulua nigro macubar, poficae virides maculis tribus baieos \& verfus apicem fafcis duabus argenteis fuluo marginatis adangulum ani coenntibus.

Eamms 321-22. PAPILIO P. R. alis tricudatis fufcis: fubtus albis flrigis duabus fulus. 
Papilio Faumus Drury Inf. 2. tab. I. fig. 4.5. Cram. Inf. 4. tab. 39. fig. B. C.

Habirat in sierra 1 con. Africae.

Alae polncae firiga pottica alba. Caudae all ie tirign poftica fubtus marginalis.

322-23. PAPILIO P. R alis fubtricandatis Pelion

cocruleis auro juncearis: limbo fufco.

Papilio Pelion Cramer Int. i. tab.6. fig. E. F.

Habitar in America meridionali.

Statura praecedentium. Alae tricaudatac canta interiori breullima, coeruleae punctis aureis numerofis limboque fufco. Subrus fufcac abo falci cae bafi obfcurores, anticis puncto parno coccineo.

$322-23$. PAPILIO i. R. alis fubtricaudatis Anms

coerulcis limbo arro: fubrus nigris albo-

falciatis.

Papilio Aunus Cram. Inf. 2. t.23. fig. E. F.

Habitat in Curacao Americae.

Paruus, alae tricaulatae cauda inrerme. dia longiore ante apicem alba.

$3=3-24$. PAlll IO L.R alis bicaudatis coe (Fany-

ruefrentibus limbo fufco: fubtus bafi vi. medts

refcentibus punctis aureis.

Papilio Gumbócs Cram.Inf.4.tab.4o.f.C.D.

Habitat in India.

Alac knja coeruleae, nitidae puncto centrali anticaram limboque omni fulcis. Sub. tus comnes bafi virefcentes punctis numero. fis anreis. In medio anticarum ftrigas duae alba 
albæ vnicaque purpurea albæ adnata, poficarum fafcia alba, purpurea viridisque ap. proximatx. Margo pofticus fufus punctis aliquot anreis ad angulum ani.

Mars $324-25$. PAPILIO l'. R. alis bicandatis fufcis: pofticis macula rufa, fubtus punctis duobus farciaque obliqua alba.

Habitat in America meridionali Dom. Spengler.

Statura pracedentium. Antennæ albo nigroque annulate claua apice ferruginea. Alix anticre fufce macula media obfuriore. Subtus cinerex fafcia obliqua alba. Pofticie caudarx: caudis fufcis apice albis; exteriore multo breuiore, fufce macula ante marginem rufa. Subus cinerere pundis duobus farciaque obliqua aibis. Macula magna ruf ante angulum ani.

Celerio

$3-25$. PAlLIO. P. R. alis bicaudatis al-

bis, fubtus fafciis fufcis albisque alternis; angulo ani ocello triplici rufo.

P.pilin Celerio Cram. Inl. 3. tab. 3r.fig.C.D. Habitat Surinami.

Ale fupra albe anticis apice ponticis margine punctato nigris.

Tele- $325-25$. PAMLLIO P. R. alis bicandaris coemus ruleis nitidis: potticis fubtus frriga mar. ginali angulata atra.

Papilio Telemus Cram. Inf r.tab.4. fig. D.E. Habitat in America meridionali. 
Alae omnes coerulere anticis fupra macula media atra. Caudio duæ nigre. 325-26. PAPILIC P. R. alis bicaudatis fu. Hessoss pra fufcis immacularis: poíticis fubtus angulo ani fifcis tribus coeruleis.

Papilio Hemon Cram. Int. 2. tab. 20. fig.D.E. Habitar Surinami.

Alæ fubrus pallidiores anticis frrigis dua. bus dimidiatis nigris, poftıcis puncto bafeos atro pupilla lareraii alba, verfus angulum ani ftriga incurua abbreuiata alba \& verfus marginem thigx tres nigra, quæ ad angulum ani fafciis tribus coeruleis feparantur.

$34 \mathrm{I}-42$. PAPILIO P. R. alis caurdatis albis zinus

apice fufcis: fubtus albo atroque fafciaris;

anguio ani punctis tribus albis.

Habitat Surinami Dr. Schulz.

Alr anticre albre pundto medio apiceque late furce. Subtus fafciis quatuor albis atrisque alternis, intermediis duabus atris abbreuiatis. Margo ipfe ftriga abbreviata, ferruginea. Pofticæ albæ margine nigro ftriga alba. Cauda nigra terminatur puncto albo. Subtus fafciis fex albis atrisque alcernis adangulum ani coeuntibus. Fafcia exterior \& interior ferruginex. Angulus ani ater punctis tribus albis.

348 - 49. PAPILIO P. R. alis integris fupra Icavs

albis fafciis fufcis: fubrus fafciis albis nigrisque alternis. 
Patilio Philemon Cram.Inf. 2.tab.22. f.G.H. Habitat Surinami.

- Alre fupra albre fafciis quaruor puneroque intra primam \& fecundam fufcis. Subrus fafcis albis nigrisque alternis ponica nigra in alis pofticis punctata.

Hippo- 353 - 54. PAllLIO P. R. alis integris marthoe gine albo: fubrus cinereis punctis ocellaribus numerofis.

Pupilio Hippotboe Linn. Sylt. Nat. 2. 793. 254. Fn Sv. 1046.

Roef. Inf. 3. tab. 37. fig. 6.7.

De Geer. Inf. 2. tab. 2.

Habitat in Europa pratis fyluaticis.

Color alarum fuperne nimis variat. In. terdum omnes fuluae margine maculaque enteriarum nigris, interdum fufce fuho ma. culatx nigrogue punctatix, interdum fuluo coerulefiences nigro punctatio. Margo vero femper tenuifime albus.

Cafius $360-6$. PAMLLIO P. R. alis integris fupra albis: anticis limbo fufco, potticis fimbria punctara.

Papilio Caffi Cram Inf.2.tab. 23. fig. C.D.

Habitat Surinami.

Paruas. Alæe anticic limbo maculisque duabus difci fuficis. Macula balicos coerulea. Poltice albre margine potico pundisquinque nigris, qua itrigis dusbus induduntur. Subrus omnes albce nigromaculatce margine portico nigro punctato.

381 . 
38r. PAPILIO P. V. alis caudatis atris fafciis Licars duabus albis: angulo ani rubro bimaculato. $\sqrt{\text { is }}$ Habitat Surinami Dr. Schulz.

Alre antice atræe fafciis duabus albis, fubrus concolores lineola punctoque bafeos ru. bris. Pofticæ atre fafciis duabus obliquis al. bis pofteriore obfoleta. Cáuda elongata obtufa punctis aliquot obfoletis albis. Adangu. lum ani puncta duo rubra. Subtus concolores, at intermaculas anales puncta duo parua cyanea.

381-82. PAPILIO P. U. alis candatis $\mathrm{fu}$ - Melifcis: anticis ftriga pofticis duabus fangui. boeus neis, fubrus cyaneo nitidillimis.

Habitat Surinami Dr. Schulz.

Alce anticre furce Atriga obliqua fanguinea. Subtus cyanex nitidillimie fafcia margineque atris. P'oftica fufce trigis duabus rubris, quarum anterior marginem exteriorem, pofterior interiorem haud attingit. Subtus cyanex fafcia lata atra. Angulus ani puncto rubro alboque.

391-92. PAPILIO P. U. alis rotundatis in Ara

tegris fufcis: poficis fubtus grifeis ma. cinthe culis ocellaribus albis.

Habitat in Auftria Dr. Schulz.

Antennx albo nigroque annulatx clana ouata ferruginea. Corpus fufcum fubtus albicans. Alre anticæ fufer litura parua flauefcente ad marginem crafliorem. Subtus fu. 
fcr apice flauo maculatæ. Pofticæ fupra fufcæ, immaculatá, fubtus cinereæ maculis ocellaribus albis duodecim annulo nigro cinctis 2. 4. 6. vlimis fex confluentibus.

Menip-397-98. PAPILIO I. U. atis integerrimis pus

ftriatis fulcis: facia lata aloa.

Habitat Surinami Dr Schulz.

Antenne nigra clatuacumisata. Corpus niprum. Ale antice fupra ftriate, fifice faficia media lata alba puncto centrali fúfco. Subus concolores at magis aluidce. Polticre Atracie fufce fafcia latiore alba, immaculata, Subrus albicir apice fufce.

m $f_{c a}$ 2-3. SPHNX alis angulatis obfouris puncto atro: pofticis atris fatcia fulur.

Habitat in Anerica meridionali D. Spengler.

Statura S. lugutsis. Ala anticæ cinerere fufco repando fafciatr. In ne bio alie punctum atrum (interdun duo.) Sustus oblicuregrifez. Polticie fupla atrice fatila lata fulua, qua marginem tenuiorem hou artingit. Angulus ani cinereus litura tuilua. Subtus grifex, immaculate.

fene. $3-4$. SPHINX alis erofo dentatis fufco aufrrina res: maculis duabus feneftratis.

Spibinx fencftrina Schiefferm rodr. 44 .

Habitat in Aufria Dr. Schulz.

Magnitudo maioris Mufcre. Corpus fufoum paipis albidis. Abdomen cingulis duobแร 
bus albis anteriore renuiore. Alx concolores fufcn aurea, nirida punctis flauis fparfic。 In nedio maculæ duæe approximatæ feneAtratæ in pofticis fere fafciam conftituenres. Margo polticus niger maculis duabus abbis. 6-7. SPHiNX alis dentatis nigris frigis Cacus

tribus approximaris pallidis: polticis Hauis nigro Atriatis.

Spbinx Cacus Cram. Inf.4 tab. 46. fig. E. Habitat Surinami.

Magnirudo S. Ello. Alæ anticæe dentatæ nigra Anigis rribus incuruis pallidis. Pofticæ flaux margine poftico frrisque octo bafin haud attingentibus nigris. Corpus nigricans abdomine cingulis cinereis nigrisque aliernis.

$16-17$. SPHIN alis integris grifeis: pun plebejs cto medio albo, abdominis lateribus albis nigro marginaris.

Habitat in America Infula 1t. Crux D. Spengler.

Magnirudo S. Pinaitri. Alæ anticæ grifex lineolis aliquot indiftinctis nigris punctoque medio albo. Pofticæ vtiomnes fubtus nigræ margine poltico tenuifime albo. Abdomen fupra grifeum linea dorfali nigra. Latera alba vtrinque nigra. Subtus album linea punctorum fuifcorum.

I7 - I 8. SPHINX alis inregris virefcentibus Galliz vitta alba: pofticis pallidis bafi ftrigaque atris, antennis furcis. 
Spbinx Gallii Schieff. Prodr. pag. 42. Sploinx fexta Scheff. icon. tab. 78. fig. r. 2. Spbinx francofuriana Pallas spicileg. XI.

Roef. Inl. 3. tab. 6. Larua.

De Geer Inf. 2. p. 236.

Habitat in Germania Galio.

Nimis affinis S. Euphorbice. Differt tamen antennis vix apice albis, abdominis punctis dorfalibus albis alisque fubtus obfcurioribus.

Pluto 22-23. SPHINX alis integris virefcentibus: pofticis fufcis fafcia fulua, abdomine fubrus aureo.

Habitat in America meridionali D. Spengler.

Corpus medium fupra virefcens capite thoraceque linea laterali cinerea. Abdomen linea dorfili punctorum aureorum. Pectus \& abdomen fubtus aurea. Alæe antica fupra virefientes obfcuro fubfafciata lituraque apicis alba. Subtus virefcentes bafi fubflauefientes. Poftica fufcac falcia lata fulua, fubtus flauefcentes ftriga fufca atomisque vire. fcentibus. Apex alæ virefcit.

Melas 2-3. SESIA alis feneftratis: margine atro, abciomine cyaneo bafi atro: cingulis tribus : flauis.

Sphinx Melas Cramer Inf. 4. tab.45.fig. G. Habitat Surinami.

Magnitudo S. Hylas. Antennæ fubfiliformes. 
formes. Caput flauum. Thorax niger lineis duabus thoracem cingentibus flauis. Abdomen bafi atrum fafciis tribus flauis, apice cyaneum fegmentorum marginibus atris. Alæ omnes feneftratæ margine poftico atro. I-2. ZYGAENA atra, alis anticis viridi-Pythia bus maculis tribus oblongis approximatis fanguineis, pofticis rubris.

Sploinx fecunda Schæế. Icon. tab. I6. f.4.5. Habitat in Germania Dr. Schulz.

Affinis Z. Filipendulæ. Antennæ clauatæ, atræ. Corpus villofum, atrum. Alæ anticx virides maculis tribus elongatis, approximatis, fanguineis ad bafin 2. 1. Pofticæe fanguineæ margine cyaneo. Subtus concolores.

I-2. ZYGAENA cyanea, alis anticis vi- Fuluia ridibus punctis quinque rubris, pofticis fanguineis immaculatis.

Spbinx quinta Schæeff. Icon. tab. 7 I. fig. I. Habitat in Auftria Dr. Schulz.

Nimis affinis Z. Fiiipendulæ. Antennæ extrorfum craffiores, atræ. Thorax cyaneus, albo maculatus. Abdomen coerulefcens, immaculatum. Alæ anticæ virides maculis quinque farguineis duabus bafeos connatis. Margo parum albicat. Pofticæ fanguineæ, immaculatæ. Subtus concolores. 5-6. ZYGAENA atra, alis anticis cyaneis carnio. punctis quinque fanguineis fubocellatis, lica polticis rubris: limbo nigro.

$$
\mathrm{S}_{2} \quad \text { Sphinx }
$$


Sphinx earsiolica Scop. Ent. 478 .

Sphinx Jeptima Schrif. Icon. tab. 8 I. f. 4.5 . Habitat in Auftria.

Thorax albo maculatus. Macula alarum annulo albo cinctze, poftica transuería. Ab. domen atrum rarius cingulo poftico fanguineo.

Bro. 10-10. ZYGAENA ferruginea, alis hya. sus linis: margine omni nigro.

Sphinx Bromus Cram. Inf. 3 tab. 35. f. G. Habitat Surinami.

Antennæ pectinati: Corpus totum obfcure ferrugineurn, immaculatum. Alee hyaline margine emni nigro.

Hely. I4-15. ZYGAENA atra, abdomine lineis nus tribus aureis.

Sphisex Helymus Cram. Inf. r.tab. 2. fig.D.E. Habitat Surinami.

Antennæ vix pectinatæ, atræ. Alæatræ, fupra immacularæ, fubtus linea pofticarum bafeos aurea. Abdomen atrum fupra lineis tribus latis aureis.

Evi. 16-17. ZYGAENA virefcens, alis poftithia cis atris: fafcia alba, antennis pectinatis.

Habitat Surinami Dr. Schulz.

Antennæ fufcr pectnatæ. Caput \& thorax obfcura. Abdomen virefcens vtrinque puncto bafeos albo, fubtus ftriis duabus albis. Alæ anticæ fupra virefcentes, immaculatae, fubtus virefcentes margine interio: 


\section{म․ำ}

ralbicio. Pofticre fupra atræe vitta lata alba. Subrus virefcentes, immaculatie.

19) -20. ZYGAENA alis atris: anticis fa. Ery fira obliqua reftacea.

nis

Habitat Surinami Dr. Schulz.

StaturaZ. glancopis. Antennæarræ. Corpus arrum, immaculatum. Alæ anticæ atræ faficia obliqua reftacea. Poltico atrx, immaculate. Subtus concolores at bafi coeruleo nitidifima. Abdomen fubrus lineis du. bus punctorum albidorum.

aI-22. ZYGAENA alis flau hyalinis api- cmusa

ce fufcis, corpore auro maculato.

Spbinx courctuta Cram. Inf.I. tab. 4. f.F.G.

Drury Inf. 2. tab. 17. fig. 2. Famina.

Seba Mur. 4. tab. 25. fig. 17. Famina. Pall. Spicil. Zool. I I. tab. 2. f. 8. Mas.

Habitat in America meridionali.

Media. Antennæ pectinatæ. Corpus fufurn linea thoracis dorfali maculisque plurimis abdominis aureis. Alo concolores flawo hyalinge margine fufco. In anticis etiam macula centralis fufca. Cauda maris colpore longior, villofa.

3-9. BVMBYX alis parencibus caudatis semverficoloribus puncto feneftratis: caudis ramis longifinis.

Phalaena Semiramis Cram. Inf. z. rab. I3. F.A.

Habitat inSacharo America meridionalis. Magna. Ala bafi Alaux apice verficolo$S_{3}$

res 


\section{8}

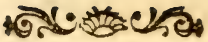

res puncto triangulari feneftrato. Pofticæ bafi fuluæ puncto orbiculari feneftrato. Cau. dæ corpore triplo longıres, fufcæ apice albæ. Penelo. $10-I$ I. BOMBYX alis patentibus flauepe

fcentibus fufco irroratis ocelloque centrali feneftrato.

Pbaldena Penelope Cram. Inf. 4. tab.45. f. A. Habitat in India.

Magna \& craffa. Antennæ pectinate apice feracer. Thorax ferrugineus. Abdomen flauefcens fegmentis bafi ferrugineis. Alæ omnes concolores, flauæ fufco irrara. tæ. Striga angulata bafeos \& alia reeta transuerfa fufcæ. Macula centralis feneftrata annulo nigro cincta \& præterea in antıcis puncta duo parua \& fere obfoleta feneitrata.

Thyr- 10-II. BOMBYX alis patentibus grifeis hea

ftrigis albis ocelloque centrali atro: pupilla feneftrata.

Pbalaena Thyrrbea Cram. Inf. 4. tab. 46. f.A. Habitat ad Cap. B. S.

Corpus magnum, ponderofum. Antennæ pectinatæ. Thorax grifeus antice ftriga alba. Abdomen fubreftaceum. Alx anticx grife $x$ Atrigis duabus albis: anteriore bafeos valde flexuofa, polteriore verfus marginem pofticum duplicata, vndata. Inter ftrigas ocellus magnus ater iride alba pupillaque feneftrata. Pofticx bafi teftacex apice grifex ftrigis duabus, altera bafeos angulata, altera 
verfus apicem duplicata, vndata. Ocellus inter firigas ater iride flauefcente pupilla feneftrata.

60- ́́. BOMBYX alis deflexis flaniffimis: fulphrs-

ftrigis duabus obliquis obfcurioribus.

Habitar in Germania de Hartorf.

Magnitudo B. curtulx. Corpus rotrim flauifimem. Als anticx itidem flaur ftrigis duabus, quarum anterior a medio marginis craflioris verfus bafin marginis tenuiuris, polterior ponemedium marginis crantoris ad angulum ani ducitur. Alde poftica im. maculatx.

$64-6 ;$. BOMBYX alis deflexis fubdentatis Gnomis

cinerco albis: vitta marginali atra; macula alba.

Habitat Hamburgi Dr. Schilz.

Statura \& magnitudo omnino $\mathrm{N}$. Verbafci. Antennx filiformes, vix pecinat $x$. Thorax grifeus ftriga antica pofticaque nigris. Alx antica nitiex ad marginem tenuio. rem cincre $x$ vitta atra, in qua a margine poltico macula arra afcendit. Verfus apicem margrinis craffioris macula fufca lineolis duabus arris maculaque alba. Subrus grifex. Poftica albidx, fubrus macula ad marginem exteriorem fufca. Abdomen grifeum ano albo. 69-70. BOMBYX alis comprenio adfcen-comdentibus niueis: macula conmuni fufca, prefje centrali grifea: lunula alba.

$$
\mathrm{S}_{4} \text { Habi- }
$$


Habitat in Germania.

Parua in hoc genere. Antennx fimplices, fufcx. Alx valde compreffx poftice adfeendentes, niuex anticis macula communi magna fufa \& in medio macula magna transuerfali grifea lunula parua niuea. Verfus marginem pofticum lunulx fufcr \& margo ipfe fufcus. Alx pofticx niuex. Subtus anticx grifex pofticx niuex.

83-84. BOMBYX alis deflexis flanis riullis punctisque atris: pofticis fufcis.

Pbaldena fulpbouralis Linn. Syft. Nat. 2. pag. 881. 333.

Scharf. lcon. 1. tab. 9. fig. I4. 15. mal. Habitat in Germania.

Statura B. Plantaginis. Al $x$ antic $x$ deflex $\boldsymbol{x}$ Aauefcentes lineis duabus latis apicem haud attingentibus ad marginem renuiorem, in medio puncta duo \& ad marginem craflio. rem tria, verfus apicem denique ftrigg dus e punctis concatenatis atris. Poftica fufcx margine tenuiffime albo. Subtus omnes variegatx.

T\% $86-87$. BOMBYX alis defexis? cinereis gawnius macula media atra: linea ramum exferente alba.

Phalaena Tarquinius Cram. Inf. I. tab. 4. fig. B. C.

Habitat Surinami.

Sequenti duplo minor. Antennx pesti- 
natx. Caput \& thorax atra, immaculata. Abtomen rufum. Alre antica cinereze atomis paucis nigris maculaque media trangulini atra, qua linea vniramofa alba in tres diuidirur. Poftic rafie macula media atra fafingque duabus obfcurioribus.

$86-87$. BOMBYX alis deflexis? anticis ni- Tar. gris linea hamata inter ftrigas duas albas. quinis Phaluena Tarquinia Cram. Inf.r. tab.4. fig. A. Habitat Surinami.

Afrinis pracedenti differt tamen colore alarum anticarum abdomineyue nigro annulis rubris.

95-96. BOMBYX alis incumbentibus gri-Colon feo-fufcis: punctis duobus nigris diftantibus.

Habitat in Germania de Hattorf.

Magnitudo B. Furcula. Antenna nigra pectinata. Palpi nigri. Thorax hirtus gri1eus. Pectus albidum. Ala planæ incumbentes obfcure grifece punctis duobus nigris altero ad bafin altero pone medium. Subtus oblcure puncto medio fufco.

99-100. BOMBYX alis conuolutis atris, Umber

fronte abdomineque fuluis.

Pbalana Uinber Cram. InÍ. 2. tab. I 5. fig. F. Habitat Surinami.

Antenne pectinate. Alæ atræ pofticis bafi pallidioribus.

$106-7$. BOMBYX alis incumbentibus atris arm-

niueo macularis, tibis albo annulatis. lut

$$
\text { S } 5 \text { Habi. }
$$


Habitat Hamburgi Dr. Schulz.

Caput \& thorax nigra albo variegata. Abdomen grifeum. Alie antice atre bati macula alba nigro punctata, tun: litura marginis tenuioris ftrigis duabus nigris. In medio fafcia lata ad marginem tenuorem interru. pta ad craffiorem bifita alba. Verius apicem ftriga vndata, que ad marginem crafo fiorem macula ouata terminatur. Subtus fufcæ margine albo punctato.

fulmi- 106 - 7. BOMBYX alis incumbentibus grinea feo fufcoque variegatis, thorace ancice al. bo ftriga nigra.

Habitat in Germania Dr. Schulz.

Antennæ pectinatæ ferrugineie. Thorax grifeus antice albus ftriga tenui nigra. Ad latera vtrinque lunula alba. Ala anticæ bali albidre lituris nigris, tune grifex, in medio fafçia lata fufca, qux antice pofticeque a firiga vndata alba includitur. Micule ordinariæ albidæ in hac fafcia. Alæ apice grifeæe ftriga vadara nigra. Pofticre grife pe.

Boleti $21-22$. NOCTVA lauis alis depreris planis fufco irroratis, antennis pectinatis. Habitat Kilonii in Boleto verficolore. Parua ftatura Tinere at palpi huius generis. Antenne parum pectinatie. Ale antica cinerex atomis maculisque fufcis numerofis, pofticæ fufcæ margine craffiori cinereo.

vire. 27-28. NOCTVA leuis alis defexis virefcentibus: ftrigis tribus faturarioribus. 
Habitat in Americæ Infula ft. Crux.

Media. Corpus virefcens. Antennæ linguaque forruginex. Palporum fuperior articulus brens. Alæ anticæe virefentes ftrigis tribus obliquis faruratioribus. Poftica albx margine poltico litura ferruginea. Subrus omnes albæ.

33-34. NOCTVA lruis, alis deflexis ci- Robonereis: Atrigis duabus vndatis albis, ma-ris cula centrali niuea: lunula nigra.

Roef. Inf. x. Phal. 2. tab. 5o.

Habitat in Germanize Quercu.

Media. Antennæ fetaceæ, fimplices.

Strigae alarum albæ fufco marginara.

Larua glabra viridis verinque liners duabus longitudinalibus flauis.

Puppa nigra.

42-43. NOCTVA lacuis, alis deflexismonilis

brunneis: punctis quatuor approximatis

albis, antennis pectinatis.

Habitat in Anglia.

Antennæ ferruginece, pectinatæ. Caput \& thorax ferruginea. Abdomen pallidum. Ala anticæ brunneæ puncto medio obfcuro $\&$ ad marginem tenuiorem puncta quaruor approximata transuerfim pofica alba, quorum r. 3. minora. Verfus apicem itidem puncta quatuor flauefcentia at obfcleta. Poftica pallidiores fubtus ftriga obfcuriore. 50-5 I. NOC'TVA læuis, alis lanceolatis lances. cinereis: anticis fufco irroratis. lata Habi. 
Habitat in Germania Dr. Schulz.

Antennæ longitudine corporis nigra ap!ce albæ. Alæ lanceolatæ acutx, cinerere anticis fufco irroratis, pofticis immaculatis.

assi- 67-68. NOCTVA criftata, alis incumasis bentibus: bafi fufcis; ftrigis vadatis ful. uis, apice cinereis.

Roef. Inf. 3. tab. I r.

Habitac in Germaniæ Salice.

Margo pofticus alarum nigro punetatus.

Larka virefecons lineis quinque longitudisalibus ftrigisque albis.

Pupps vix folliculata, brunnea.

eque II - I 2. PHALAENA pectiricornis, alis pravia angulatis: anticis fufcefcentibus fácia lata flaua, pofticis flauis.

Habitat Hamburgi Dr. Schulz.

Alar anticre angulate fufre in medio farcia faua. Poftice angulato - dentate flaure apice obfcuriores. Subrus antica flatuefcentes. puncto medio nigro, apice obfcure margineque flato variegato. Pofticre obfcurce bafi pallidæ punctoque medio nigro.

hyor - I8. PHALAENA pećtinicornis, alis sin angulatis viridibus: anticis ftrigis tribus fulcis.

Pladuera lyrias Cram. Inf. r. tab. Io. fig. E. Habitat Surinami.

Anus rufo barbatus. Alæ fubtus ferria. gineæ.

$$
15-16 \text {. }
$$


15-16. PHALAENA peetinicornis alis monidentatis viridibus: friga punctorum ni- laria grorum, pofticis punctn duplici albo.

Habitat in India Dr. Forhergill.

Alx dentatie virides. Anticre in medio puncto nigro, pone medium ftriga e punctis nigris. Pofticx in medir puncto gemino albo \& apice ftriga e punetis nigris. Subrus omnes plumber. Firmina fere feticornis.

24-25. PHAL.AENA pectinicomis, alis roreo flauis fufco irroratis: anticis apice nigris. rie

Habirat in Europa.

Alae omnes vtrinque flatae atomis pluri. mis fucicis irroratae. Margo pofticus alae anticae fupra late niger.

Variat rarius absque margine alarum anticarum.

26-27. PHALAENA pectinicornis, alis profsJutefcentibus atomis ftrigisque tribus fu-piaris fcis: tertia maculari.

Pbalaena profapiaria pectinicornis alis lu. tefcentrbus: fafciis vtrinque duabus faruratioribus Linn. Syft. Nat. 2. 864. 222. Fn. Sv. 1252 . Clerk. Icon. Phal. tab. 3. f. I.

Vdm. difl: 66.

Habitat in Europae nemoribus.

Striga anterior reliquis latior, media ad marginem craffiorem bifida, tertia macularis. Margo alarum punctatus.

$$
30-31
$$




\section{6}

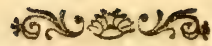

Birta. $30-3$ r. PHALAENA pectinicornis, alis yia cinereis: ftriga fufca vndata albae innata.

Habitat in Europae pratis lyluaticis.

Antennae pectinarae, fufcae. Alae anticae cinereae in medio itriga obfoleta fufca \& verfus apicem alia dentata albae innata. Margo pofticus nigro punctatus.

ditaria 33-34. PHALAENA pectinicornis, alis viridibus: maculis marginalibus ferrugineis.

Habitat Hamburgi Dr. Schulz. Antennae pectinatae, cinereae rachi alba. Alae anticae virides ftrigis obfoletis albis \& macula magna ferruginea ad apicem marginis tenuioris. Polticae rotundatae virides margine albido ftriga tenuiffima nigra. Ma. cula magna ferruginea ad angulum ani \& altera ad apicem marginis tenuioris. Subtus omnes pallidae.

margi.40-4I. PHALAENA pectinicornis, alis naria albidis puluerulentis: omnibus ftriga marginali punctorim nigrorum.

Habirat Hamburgi Dr. Schulz.

Duplex eft varietas; altera alis albidis an. ticis ftrigis tribus pofticis ftriga punctoque centrali fufcis; altera alis flauidioribus apice obfcuris, pofticis puncto centrali fufco. Conveniunt veropunctis nigris marginalibus diftinctis vtrinque in omni ala.

Grnara 47-48. PHALAENA feticornis, alis angulatis 


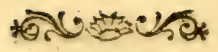

\section{7}

latis furco albo nigro fuluoque variis: pofticis fubtus albis.

Pbuluche Cynara Cran.Inf.2.tab.14 fig.C.D.

Habitat Surinami.

49-io. PHALAEN a feticornis, alis emar-finuata

ginato-grifeis: punctis duobus fufcis in-

ter ftrigas albidas.

Habirat in Germania.

Affiuis P. falcatae. Corpus grifeum capite fufco. Alae anticae apice emarginatae grifeae ttrigis duabus flexuofis altera ante, altera pone medium albis vtrinque fufco terminatis. In medio puncta duo approximata, fulia. Emarginatura alae fulua. Pofticae grifcae ftriga alba punćtoque centrali fufco. Subrus omnes rufefcentes puncto nigro. 49-50. PHaLAENA feticornis, alis angu-manton-

latis fufcis: fafcia obfcuriore; anteriarum ta puncto ocellari atro.

Pbalacna Manto Cram. Inf. I. tab. Io. fig. F. Habitat Surinami.

Alae valde angulatae. In centro alae an. ticae punctum atrum iride ferruginea. 54-55. PHALAENA feticornis, alis den-obfcastatis grifeis: ftrigis pallidioribus vndatis ratas obfoleris.

Habitat in Germania Dr. Schulz.

Media. Alae omnes valcle dentatae, grifeae ftrigis indiftinctis pallidioribus. Bafis alae anticae fufcefcit.

$$
55-56
$$




\section{8}

mitata 55-56. PHALAENA feticornis, alis fubdentatis obfcure grifeis: fubtus pallidio. ribus puncto farciaque furcis.

Habitat in Germania Dr. Schulz.

Parua. Alae omnes fupra vadatae, obfcure grifeae punctis aliquot minutiflmis fere obfoleris nigris. Subtus omnes cinereae puncto centrali tafciaque vodata fufcis.

eque- 6;-66. PHALAENA fericornis, alis omnifrara =-bus nigiris: fafcia fulua.

Habitat Hamburgi Dr. Schulz.

Antennae craffae, filiformes, nigrae. Cor: pus nigrum. Alae rotundatae, concolores, nigrae fafcia lara fulua, quae tamen marginem exteriorem alae anticae haud attingit. soeris. 67-68. PHALAENA feticornis, alis obfcudata re viridibus fufco fafciatis fafciisque dua. bus coeruleis.

\section{Habitat Hamburgi.}

Antennae fufcae. Caput \& thorax vire. fcentes. Abdomen cinereum. Alae anticae obfcure virefcentes fafciis ftrigisque furcis. Ad bafin faficia obliqua ad marginem tenuiorem cillarata \& in medio altera latior laete coerulefcentes. Pedes pallidi tibiis nigro annulatis.

grifasa $72-73$. PHALAENA feticornis, alis orifeis: anticis ftriga fafcia punctoque fufcis. Habitat Hamburgi Dr. Schulz. Antennae cinereae. flae anticae grifeae itriga 
ftriga bafeos fafciaque lata poftica fufcis. Punctum fufcum ante fafciam. Ala pone fafciam obfcursor. Pofticae grifeae fafcia lata fufca, in qua punctum nigrum \& pone fafciam obfcuriores. Subtus omnes grifeae punto centrali trrigaque poftica fufcis.

77-78. PHALAENA feticornis, alis plum-fmbeis: fafcia lata fufca flawo marginata. "briata Phaluena Xull Schaeff. Icon. 1. tab.80. f.6.7. Habitat in Germania Di: Schulz.

Punctum centrale partum arrum in fafcia lata fufar

77-78. PHALAENA rcticornis, alis grifeo quadrifufcis: fafcia lata nigra; puncto fubocel fufcialari nigro. Linn. Fn. Sv. 1253.

Hebitar in Europae pratis fuluaticis.

Alae fubrus albidae puncto centrali ftrigisque nigris.

79-80. PHALAENA feticornis, alis albi-rinuludis: fafciis duabus nigris: pofteriore ter-to minali.

Habitat in Germania Dom. de Hattorf. Caput \& thorax fufca. Abdomen cinereum dorfo fafciculis duabus erectis, brenibus. Alae anticae bafi albae litura marginis craffioris fufca. In medio fafcia fubfinuata fulca, pone hanc alae albae litura marginis craflioris obfoleta, fufca. Margo pofticus fulcus ftriga punctorum nigrorum. Pofticae obfcurae. Subtus anticae fufcae maculis ob- 
foletis albis, pofticæ albæ puncto centrali fafciisque duabus fufcis.

Cen. 83-84. PHALAENA feticornis, alis fertumno- rugineo fufcoque variis: fafcia media grirata fea C. atro notara.

Phalaena centumnotata Naturf. 6. tab. 4. fig. 4 .

Habitat in Germania.

Ruftica media ftatura P. Chenopodiatæ. lumula. 84-85. PHALAENA reticornis, alis fufcis *a poftice ftrigis vndatis flauis.

Habitat in Germania Dr. Schulz.

Media. Corpus fufcum. Alæ anticæ fufcæ margine antico flauo punćtato. Verfus marginem pofticum ftrigæ tres e lunulis flauis, guarum anterior macula maiori terminatur. Margo ipfe flauo punctatus. Pofti. cæ fufcæ apice ftrigis tribus vndatis margineque poftico punctis flauis. Subtus omnes bafi cinereæ, a pice obfcuriores ftrigis pallidis. media. $89-90$. PHALAENA feticornis, alis omta nibus grifeo vndatis puncto centrali fufco margineque nigro punctato.

Habitat in Germania D. de Hattorf. Affinis P. punctatae. Alae omnes cinereae ftrigis vndatis obfoletis obfcurioribus. ceitra- 90-9I. PHALAENA feticornis, alis flameth fentibus fufco vndatis : anticis puncto centrali nigro.

Habitat Hamburgi Dr. Schulz. 
Antennae breues, albae. Alae anticac flauefcentes fafciis plurinis anaftomofantibus obfcurioribus punctoque centrali nigro. Irofticae rocundarae Anuefcentes fafciis vnd ris plurimis fufcis. Subtus concolores ar obficuriores.

92-93. PHALAENA feticornis, alis om nibus purpureis maculis margineque fauis. Habitat Hamburgi Dr. Schulz.

Corpus fulicum cauda fisuefcente. Alze antica purpurere macula magna media margineque poftico flauis. Pofticæ purpurce puncto centrali margineque flauis. Subtus concolores at obfcuriores.

99-100. PHALAENA feticornis, alis atris Mclatc

albo maculatis, ano rufo.

Pbalaena Melite Cram.Inf. 2. tab. 18. fig.D.

Habitat in America boreali.

Caput \& thorax atra. Abdomen atrum albo maculatum ano rufo. Alæa fafciis anticarum tribus pofticarum duabus macularibus albis.

107-8. PHALAENA fericornis, alis flauif-eleuata

fimis fufco punctaris fubtusimmaculatis.

Habitar in America meridionali.

Paullo maior P. indicata. Corpus flauefcens. Alæ anticxe flauifima punctis nume. rofis fparfis fufcis. Pofticæ flauifimæ punctis paucioribus fulcis. Margo pofticus omnis alæ ciliis argenteis. Subtus omnes flauæ, immaculatæ.

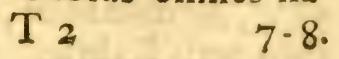


Leeara 7 -8. PYRALIS alis plumbeis: lineolis trio bus maculaque magna apicis ferrugineis. Habitat Hamburgi Dr. Schulz.

Statura P. vncanæe as duplo minor. Corpus fufcum. Ala plumber. Lineola mar. ginalis a bafi ad medium ducta, altera ver. fus marginem tenuiorem a bafi ad angulum ani ducta, rertia in medio a centro ad maculam apicis. Punetum paruum inter lineolam fecundam tertiamque. Macula apicis orbiculata.

palli. 9-10 PYRALIS alis pallide flauis: ftrigis duabus obliquis ferrugineis.

Habirat Hamburgi Dr. Schulz.

Alæ anticæ pallide flatre in medio ftrigis duabus obliquis, tenuilfimis, rufis. Pone has lineolæ aliquot abbreuiatæ, ferrugineæ.

ferua. 15-16. PYRALIS alis anticis fufcefcentina bus: macula centrali fulua, bali flauis. Habitat in Germania Dom. de Hattorf. Magnitudo P. minifiranæ. Alæ anticæ bali flauæe, medio fulcæ macula magna centrali fultua apice fere auratæ ciliis niueis ma. culaque furcg. Pofticæ cinereæ.

aspera- $35-36$. PYRALIS alis anticis bafi albis na apice fufcis fcabris.

Habitar Kilonii de Seheftedt Hamburgi Dr. Schulz.

Media. Corpus fufcum. Alæ anticæ bafi albæ, immacularæ, apice fufcæ punctis eleuatis atris fcabrix. Pofticæ cinereæ. 
35-36. PYRALIS alis anticis pallidis: duplapunctis duobus approximatis bafeos nigris. "ta Habitat Kilonii Dom. de Seheftedt.

Statura omnino P. afperanæ. Capur cum antennis cinereum. Ale albidre apice paullo obfcuriores. Ad marginem ten!ziorem bafeos ptancta duo oblonga, approximata at tamen diftincta. Ale fubrus cinerece.

47-48. PYR ALIS alis atris: macula mag- Sehe. na communi, ftrighis aromisque coerule- Rediafienti argenteis.

Habitat in Chilonii Alnetis Dom. de Seheftect Norwaous Hiftoriæ na. turalis cultor feliciflimus.

Statura \& magnitudo P. Fabricianæ. Anrennæ albo nigroque annulatæ. Thorax \& abdomen arra, immaculata. Alæ anticæ atræ bafi \& ad marginem craftiorem atomis plurimis coerulefcenti argenteis, nitidiflimis. In medio macula magna, com. munis,coerulefcens. Poftice ftrigæ duæ interruptxe ad marginem tenuiorem coeuntes coerulefcentiargentex. Margo pofticus cilis albis. Alx poftice atre margine craffiori albo. Pedes nigri tarlis albo annularis. 49-50. PYRALIS alis teftaceis fafciis pun-Schm? etisque argentêis, mediogue litura atra. ziana Habitat in Germania Dr. Schula Hamburgus.

Magna in hoc genere. Alæ anticx tefta. T. 3 cer 
cere bafi pundis aliquot argenteis, tunc fafice dua argentex ad marginem craffiorem bifita 8 inter has litura duplicata arra punctis rribus argenteis fere obfoletis, apice fafia interrupta argenrea. Alæe fubtus nigricantes maculis cuftalibus flauis. Pofticæ cinerea.

inzáa $33-54$ PYR ALIS alis bafi fufcis linea feminia circulari pallida, apice auratis: margine crantiori nigro pallidoque ftriato.

Habitat in Chilonii Quercu Dom.Lund. Norwagus Botanicus eximius \& Entomologus oculatus.

Magnitudo \& ftatura P. ftrigana. Alæe antica linea lata fernicirculari alba a bafi mar. ginis cralfioris ad medium marginis tenuioris ducta, maculam dorfalem atram includens. Pone medium fere auratæ ftriga obliqua obfoleta, argentea. Margo craffior ftrigis pallidis nigrisque variegatus apice acuminatus, ater. Margo pofticus ciliis albis. Alæe poficic fulcæ⿱亠乂.

appla. 56-57. PYRALIS alis depreffis furcis: siss punctis tribus centralibus albis.

Habitat Chilonii Dom. de Seheftedt.

Palpi recurui, medio craffiores, apice acuti. Alæe anticæ cinereo fufcæ punctis tribus minutillimis albis in centro vna pone alteram pofitis, quorum anterius puneto par. no atro terminatur. Margo exterior anice frimstus. Subtus cinereæ. 
3-4. TINEA alis oblongis grifeis, anten fniffnarum articulis duobus it ferioribus in- c.tua craffatis.

\section{Habitat Chilonii Dom. Lund.}

Antennie corpore breuiores articulo primo fecundoque incrafitis, diftinctis, pubefcentibus. Thorax poftice criftatus, cinereus. Ala grifea medio pallidiores punctis aliquot diffincris nigris.

29-30. TINEA alis niueis: arcu communi arcella ma ulisque duabus marginalibus fufcis.

Habitat in Germania Dr. Schulz.

Parua. Caput \& thorax niuea, immaculatr, pilnfa. Alæe anticæ nivex bafi ad marginem crafforem macula oblonga, ante medium arcus dorfalis communis $\&$ in medio macula cuata ad marginem crefforem fufcis. Verfus apicem puncta aliquor indiftincta fufca. Subtús cinerex.

33-34. TINEA. alis linearibus teftaceis; elonantennis mediocribus. gella Pbalacna clongella Linn. Syft. Nat. 2. 890. $285 . \mathrm{Fn} . \mathrm{Sv} \cdot 1436$.

Habitat in Europæ nemoribus.

34--35. TINEA alis anticis linearibus fu.cylin.

fcis: maculis tribus Aauis. drella

Habitat Hamburgi Dr. Schulz. Mitudia, elongara. Capur flauefcens anrennis pallidis. Thorax fucus dorfo flaue. ficente. Ala antica elongata, lineares, in-

$$
\mathrm{T}_{4} \text { cumben. }
$$


cumbentes, fuccae maculis tribus magnis, oblongis, favis. Alae fubrus fauae. $\mathrm{Pe}$ des faui fufco annu!ati.

bipnn. $38-39$. TINEA alis fufco cinereis: pundo della marginali albo.

Habitat Hamburgi Dr. Schulz.

Parua. Antennae filiformes. Capur \& thorax fufca fobfore cinerea. Alae obfcu. rae in medic lineola e punctis tribus nigris punctoque ad marginem crafiorem albo. Pofticae vii omnes fubtus cinereae.

fupho- 45-46. TINEA fufca, capite thorace alarumque bafi maculaque coftali fulphureis. Habitat in Germania Dr. Schulz.

Media. Palpi infiexi, corniformes. Caput \& thorax fulphurea, immaculata. Alae fufco auratae bafi maculaque magna coftali fulphureis. Maculam hanc circulus coeruleus niridus, tenuifimus cingit. Apex alae albicat.

Seppez $52=53$. TINEA alis auratis: frigis duabus argenteis.

Habitat in Anglia Dom. Ycats.

Minuca. Corpus nigrum antennis breuibus. Alae anticae auratae, niridae frigis duabus rectisargenteis. Potticac auro nitidae. crame-64-65. TINEA alis pallide arogenteis poftice fufcis maculis marginalibus argenteis punctogue terminali atro.

Habitat in Anglia Dom. Ycats.

Minu- 
Minuta. Alae anticae pallidae, poftice ohfurores maculis argenteis nouem, quarum 5 ad marginem craffiorem 4 ad tenuiorem. Macula fecunda transuerfalis longior fere coit cum macula fecunda marginis tenuioris. Apex alae terminatur puncto fubocellari atro. Polticae cinereae, immaculatae. I-2. ALVCITA alls pallide auratis vitca epsip. dorfali niuea: farcia aurea.

Habirat in Europa Dr. Schulz.

Parua. Alae pallide auratae vitta lata communi dorfali niuea, in qua facicia lata aurea.

9. ALVCITA alis niueis: maculis dua-miweella

s marginalibus farciaque media nigris, capire alto.

\section{Habitat in Anglia Dom. Yeats.}

Magnirido A. graneliae. Corpus cinereum capire albo. Alae anticae niveac mafula oblonga marginali baleos nigra. Inmedio falcia obliqua vnidenrata \& verfus a picem macula parua marginalis riggra. Alae pofticae cinereae.

10- - 1 . ALVCITA alis fufco cinereis pun. Cor.

ttis nigris elevaris fcabris.

Pbalacna foubsella alis albis: dorfo rigro

frriatis exafperatis, palpis fpinotis Linn.Sytt. Nat. 2.89 r.395. Fn. Sv.1446.

Habirat in Europa boreali.

Puncta alarum nigra in ftrigas difpofita.

12-13. MEMDRACIS thorace inermi fer-mutica

Ts rugi- 


\section{$29^{8}$}

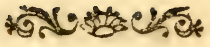

rugineo: poftice producto carinato longitudine abdominis.

Habitat in America boreali.

Corpus obtufum, ferrugineum. Thorax inermis lineis tribus cinereis, obloletis, poftice productus eleuato carinatus litura marginali ftrigaque poftica cinereis, obtoleris. Elytra oblcura. Pedes ferrugine:

coss. IO-II. TETTIGONIA atra, elytris bafi fonrca- flauo maculatis, ano flauo.

sa Habitat in India Dr. Fothergill.

Corpus paruum fupra nigrum abromine fubrus anoque flauefcentibus.

bafi flano maculata. Alæa atræ.

Quer- 36. CICADA flauefeens, elytris bafi fancus guineis, apice fufco maculatis.

Habitat in Quercu frequens in predio nobilifimi comitis Holk Ekhof dicto.

Minuta. Caput \& thorax flauefientia oculis nigris. Elytra iridem flauefientia maculis quaruor oblongis fanguineis. Apex macula magna dentata fufca. Alæa alba.

coloop- 2. SIGARA elytris tatis coriaceis fufcis: trate margine exteriori fufco.

Habitat in Chilonii aquis Dom. de Seheftedt.

S. ftriatio antinis, at quadruplo minor. Caput cum roftro famm, immaculatum. Thorax fufcus, poftice rotundatus. Elytra tota coriacea, fufca margine exteriori Haue- 
fcente. Pedrus \& pedes pallida. Abdo. men nigrum.

I-2. ACANTHIt nigra, elyrris coriaceis Zofteabdomine longioribus, apice hyalinorae ftriatis.

Habitat Chilonii ad littora maris velociffime fupra Zofteras \& Fucos curfitans. Dom. Lund.

Duplo minor A. littorali. Caput nigrum antennis breuibus oculisque prominulis. Elytra coriacea, corpore longiora apice li. neolis quatuor margineque hyalino diaphanis. Corpus nigrum pedibus fauefcentibus. 1-2. ACANTHIA aptera, elytris coria-coviaceis grileis.

Cimex grylloides apterus niger, elytris ouatis margine luteis abdomine breuioribus Linn. Sy凡t. Nat. 2. 717 . I3.

Habirat Chilonii Dom. Lund.

Magnirudo A. lectularize. Anrenna nigræ articulis duobus anterioribus craflioribus. Thorax \& elytra grifea. Corpus \& pedes atra.

135-36. CIMEX oblongus ninger, fcutel-fexgut.

lo maculisque tribus elytrorum fauis. cea

Habitat in Europa.

Caput nigrum orbita oculorum flauefcente. 'Ihorax ater linea dorfali punatoque vtringue marginali flauis. Scutclium flautum. Hiytra lauia nigra macula oblonga bafeos flaus, 
flaua, fecunda in medio marginis tenuioris \& tertia apicis quæ terminatur puncto atro. Corpus atrum.

sogeus I 38 - 39. CIMEX oblongus fanguineus, capite elytrorumque apicibus albis alis fúcis. Habitat in Germania Dr. Schulze.

Paruus. Caput albidum. Thorax, fiurellum \& elytra fanguinea. Elytra apice albida. Alrefufce. Femora fangunea. Tibice albidx nigro punctaræ.

orus I44-45. CIMEX oblongus, elyrris fufen ceus ferrugineis apice cocineis puncto atro, antennis apice capillaribus.

Cimex quatuor antennarum articulis tertius Schceff. Icon. 1. tab. I 3. fig. 2.

Habicat Hamburgi Dr. Schulz.

Capur glaucefcens antennis rufis articulis apice nigris, capillaribus. 'Thorax oliuaceus, antice nigricans. Scurellum nigrum margine rufo. Elytra obfcura, ferruginea apice coccinea puncto atro. Corpus nigrum. spifis. $147-48$. CIMEX oblongus niger, pelibus flatis, antennis incraílatis apice capillaribus.

Habitat Hamburgi Dr. Schulz.

Antennie crafle, nigra, apice capillares, pallidiores. Corpus nigrum elytris imma. culatis. Alre coerulefcentes. Pedes pall:de flauefcentes.

laters- 148-49. CIMEX oblongus niger, lateritis bus albidis. Habi. 
Habitat Kiliz Holfatorum Dom. Lund.

Antennze fetacex, nigræ. Caput nigrum orbita oculorum maculaque frontali pallidis. Thorax niger, niticus lineis tribus flauefcerribus. Scutellum nigrum linea longituciina. li flauicante. Elytra nigra lateribus aibriis. Abdomen nigrum vtringue linea laterali magna flaua. Pedes nigri.

Variut rarius thorace immaculato.

\$48-49. CIMEX oblongus viridis imma-pabulio culatus, alis hyalinis. nuts

Cimex papulinus oblongiufculus Alauefcen-

ti viridis totus Linn. Syft. Nat. 2. 727.

83. Fn. Sv. 947. Scop. Carn. 132.

Habitat in Europa pratis.

I53-54 CIMEX oblongus virefcens fafciis Tiliae

tribus fufcis: media angulata.

Habitat in Tiliis Holfatiæ Dom. Lund.

Antennix fetacex, nigræe articulo fecundo bafi apiceque pallido. Caput $\&$ thorax viridia linea laterali nigra. Scutellum viride punctis duobus paruis verfus apicem nigris. Elytra viridia fafciis tribus atris anteriore abbreuiata, media antrorfum angulata, poftica latiore. Pedes albo nigroque annulatis 162-63. CIMEX fufcus, alis abbreuiatis, Filune pedibus longiffimis.

Habitat in India Dr. Fothergill.

Antennze longitudine corporis. Thorax cylindricus, linearis, fufcus. Abdomen elon 
elongatum, cylindricum, fufcum. Pedes longiffimi pallidi annuls furcis.

einctus 5-6. REDVVIVS rufefcens, antennis pe. dibusque flauo nigroque annulatis.

Habirat in America boreali.

Roftum arcuatum, flauefcens dorfo bafaeos annuiis duobus apiceque rigris. Antennæ nigra articulo primo annulis tribus fecundo duobus flauis. Caput fianeficens vertice nigro puncto flauo. Thorax rufe. fcens antice anguftarus vtrinque dente breui, concolore, poftice dente vtrinque fere obíoleto nigro. Scutellum \& elytra rufe. fcentia, immaculata. Abdomen rufefcens margine eleuato nigro variegato. Pedes annulis nigris flauefcentibusque alternis.

Diade. I I - I 2. REDVVIVS niger, capite thorasisa ceque fpinofis. Habitat in America boreali.

Antennce fufca. Caput nigrum poftice attenuatum roftro arcuato fpinisque plurimis erectis validis armatum. Thorax niger lobo antico fpinis erectis plurimis validis armato, poftice vtrinque vnidentatus. Elytra fufca. Abdomen nigro flauoque varium margine eleuato acuto. Pedes nigri femoribus tibiisque anticis fpinolis.

Acbil. 12-13. APHIS flauefcens, abdomine vilease ridi, antennis pedibusque albidis.

Habitat in Achillæa millefolio Holfatiæ. Anten- 
Antennæ filiformes, albidx. Alæ albidx. Anus, ftylus \& cornicula albida.

12-13. APHIS fufca, abdomine virefren- Mille-

te, antennis pedibus corniculisque nigris. folis Habirat in Achillaca Millefolio Kilonii.

Caput \& thorax fufca, immaculata. Abdomen viride lateribus albido fubfafciatis. Cornicula ftylusque nigra. Pedes nigri. Alir comprelfie, obfeuriores.

I5 - 16. APHIS Fraxini excelfioris.

Fraxi:-

Aphis Fraxini nigro viridique variegata Geoff. Inf. I. 494. 2.

Habitat in Fraxini excelforis ramis.

Caput \&s thorax nigra. Abdomen viride fegmentorum marginibus nigris. Antennx pedesque variegati.

22-23. APHIS Aegopodii podagrariæ.
Aphis A gnposii Scop. Carn. $399^{\circ}$
Habitat in Aegopodii podagrarix foliis.

Corpus nigrum abdomine roftro pedibusque pallidis.

26-27. APHIS Papaueris fomniferi.

Habitat Kilix in Papauere fomnifero. weris

Caput nigrum antennis pallidis. Corpus nigrum ftylo corniculisque pallidis. Pedes nigri interdum pallidi geniculis nigris. Alre pallidre cofta fufca. luniores obfcure virefcentes.

2-3. CHERMES Pyri communis Linn. ${ }^{P} r i$ Syft. Nat. 2. 737. 4. Fn. Sv. 1004.

Habi. 


\section{4}

\section{कर.}

Habitat in Pyri communis folis.

Corpus paruum, grifeum ftriis lateralibus albidis.

Perfice 6-7. CHERMES Amygdali Perfice.

Chermes Perficx oblongus Geofir in! $\mathrm{x}$. 506. 4. Reaum. lô. I. fig: I. 2.

Habitat in Amygdali l'erfice ramis.

Corpus oblongum, ferrugineum.

I-2. COCCVS Indarum arborum.

Assi- Coccus Aonidum Linn. Syft. Nat. 2. 739.2. dum Habitat in Afiæ arboribus fempervirentibus.

Corpus minus atro purpurafcens.

Berule 6-7. COCCVS Betulæ albæ Linn. Sylt.

Nat. 2: 74.0.7. Fn. Sv. 1017.

Habitat in Betula alba.

Corpus minurum, albidum.

Carpi- 6-7. COCCVS Carpini Beruli Linn. Syft. si Nat. 2 740.8. Fn.Sv. 1018.

Habitat in Carpino Betulo.

Capres 8-9. COCCVS Salicum. Linn. Syft. Nat.

2. 741.14 .

Habitat in Salicibus.

Corpus ouatum, teftaceum.

Zofte- 14-15. COCCVS Zofteræ marinæ.

rae

Habitat in Zoitera marina maris balthici。

Corpus paruum, orbiculatum, album, depreffo-planum.

minm. 2-3. THRIPS elytris corporeque glaucis, tiljima oculis 


\section{सำ}

\section{5}

oculis fulcis Linn. Syft. Nat. 2. $743 \cdot 3 \cdot 11$. Sv. 1028.

Habirat in Europæ floribus.

r. OESTRVS grifeus, facie alba nigro-luccipunctata.

Habitat in Carolina Dom. Yeats.

Facies albida, buccata punctis aliquor nigris, nitidis. Vertex grifea. Thorax grifeus lateribus pallidis. Abdomen grifeum, nigro punctatum. Alre fufcefcentes fquamis halterum elongatis, rotundatis, fufcis.

3-4. BIBIO nigra tomentofa, abdomine anea.

aneo.

Habitat in Germania de Hattorf.

Statura omnino Syrphi ruficornis, at antennæ filatæ huius generis, nigræ, extrorfum craffiores apice fubulatæ, albæ. Labium obfcure flauefcens. Thorax tomentofus, obfcurus. Abdomen æneum tomen. to ferrugineo pubefcens. Pedes pallidi femoribus nigris.

II. STRATIOMYS fcutello inermi arro, muiv: abdomine niueo apice atro.

Habitat in Germania de Hattorff.

Statura precedentium at parua. Caput atrum fronte puncto albo. Thorax niger. Halteres albi. Abdomen niueum apice atrum anotamen albido. Pedes pallidi.

5. RHAGIO thorace lineato, abdomine ni- linont gro, alis hyalinis macula apiceque fufcis. 


\section{6}

\section{*}

Tipula lineata Syft. Ent. 754. 43.

Habitat in Anglia.

Antennæ cylindricæ, incurtix, longio: res quam in reliquis. Pedes antici elongati; nigri.

sipuli- 6. RHAGIO fufcus, alis albis apice nigris. formis

Habitar in Germania de Hatrorff.

Precedentibus minor. Antennæ breues, cylindricæ. Corpus torum cinereo fufium, immaculatum. Alex late, explanatx, albre margine poftico late nigro.

mufja. $2-3$. SYRPHVS antennis plumatis tomen tans tofus, thorace fuluo, abdomine virefcenre.

Habitat in nemoribus Holfatiæ de Se. heftedit.

Magnitudo S. bombylans. Os atrum fronte flaus. Antennæ ferruginex feta plu. mata. Thorax tomentofus, fuluus. Ab. domen ouarum, pubefiens, vireficens fufco. obfolete fafciatum. Alæ magnæe albidæ bafi parum rufefcentes $\&$ in medio macula magna coftali fufca. Pedes nigri femoribus porticis elongatis incuruis.

nfiii- II $8-19$. SYRPHVS antennis fetariis, thoformis race tomentofo flauefcente, abdomine atro: fegmento primo fecundoque albicantibus.

Habitat in Germania de Hattorf. Antennæ clauatæ ferrugineæ feta nigra. 
Labium flauum punctis tribus nigris. Tho. rax tomeniofus, flauefcens. Abdomen nigrum fegmento primo toto, fecundo baí albidis. Pedes nigri tibirs flatefcentibus. Femora poltica elongata, incurua.

$3^{6}-37$. SYRPHVS ancennis fetariis æeneus pmetal. nitens, antennis nigris. $\operatorname{lin} x$

Habitat in Germania.

Totum corpus, æneum, nitidum, im. maculatum. Antennie breues, fetarix, nigræ. Abdomen dorfo plano. Alä bafi ferrugineæ.

7-8. TABANVS oculis fafciatis niger, ab-pelludominis primo fegmento coeraleficente, cerss tibiis candidis.

Habitat in Germania de Hattorf.

Antenne porrectre, filiformes, nigr $x$. Caput nigrum fronte villofo, albo. Oculi in mortuo nigri, at fafcias obfcuriores video. Abdomen arrum primo fegmento coerulefcente macula media fupra fufca, fubtus pellucida. Reliqua fegmenca fupra immaculata fubtus vtrinque macula coerulefcente pundto paruo nigro. Pedes nigri tibïs candidis. 77-18. TABANVS oculis ... ferrugi- bicens neus, abdomine vtrinque maculis duabus flauis, feutello bidentato.

Habirat in Auftria Dr. Schulz.

Caput parum fercugineum oculis ma. gnis nunc arris. Thorax gibbus, ferugi-

$$
\text { U. } 2
$$
neus, 


\section{8}

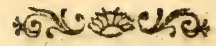

neus, vix lineatus. Scutellum concolor dentibus curobus approximatis validis. Abdomen ferrugineum fegmento primo macula dorfali fufca, fecundo tertioque vtrinque macula marginali flaua. Pedes ferruginei.

Jifpi- 17-18. TABANVS oculis ... fufcus, abnojus domine ferrugineo bafi nigro, fcurello bidentato.

Habitat Goetting $x$ Dr. Schulz.

Thorax pubefcens, fufcus, fublineatus. Scutellum bidentatum, nigrum. Abdomen ferrugineum primo fegmento nigro, reliquis ferrugineis macula dorfali nigra, lateralibus fauis obfoletis.

Ephip-5-6. ASILVS hirfutus ater, thotace bafi tium albo.

Habitat in Germania de Hattorf.

Caput hirtum, atrum antennis connatis. Thorax ater bafi hirfutie alba. Abdomen atrum. Alæ fufce. Pedes nigri plantis piceis.

sioin- IO-I I. ASILVS hirfutus ater, abdomine reis, violaceo.

Habitat in Germania de Hattorf.

Corpus rotum pilis erectis rigidis hirtum, atrum. Abdomen ouatum, violaceum, nitidum. Alae fufcr.

mfin I- 2. STOMOXYS antennis fetariis pilofa tia

rigra, abdominis fegmento fecundo tertioque rufis.

Habitat 
Habitat Hamburgi Dr. Schulz.

Corpus Mufcæ brafficariæ fimillimum, at roftrum huius generis. Caput flauefcens antennis nigris. Thorax pilofus, niger, fublineatus. Abdomen cylindricum, nigrum fegmento fecundo tertioque rufis linea dorfali nigra. Alæ bafi parum rufefcentes fquama halterum fornicata, lactea.

3-4. EMPIS nigra, tarfis anticis incraffa- maura

to ouatis.

Habitat Chilonii fupra aquas ftagnantes choreas innumeras inftituens.

Hauftellum rećtum vix inflexum longitudine probofcidis. Vagina emarginata. Antennæe articulis inferioribus breuiffimis, rotundatis, vltimo longiori fetaceo. Corpus nigrum, immaculatum. Pedes nigri tarfis anticis incraffatis, ouatis. Alæ albæ cofta dimidiata fufca.

Famina pedibus fimplicibus.

II - I2. PEDICVLVS abdomine margina-Buteo.

to: fegmentis vtrinque punctis duobus $n$ is impreffis.

Pediculus maximus Scop. Ent. 1036.

Habitat in Falcone Buteone.

Corpus fufcum. Thorax læuis lineis tribus longitudinalibus nigris. Abdomen ouatum. 16-17. PEDICVLVS albus, abdomine Orioli

linea dorfali fufca.

Pcdiculus dolichoceplatus Scop. carn. I039.

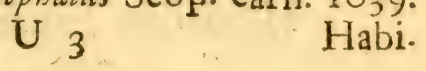


Habitat in Coracia Oriolo.

Glaber, lauis, albus. Caput elongatum. Phofina 2 - 30. PEDICVLVS capite ouato mag. $n$ i no, abdomine globofo obtufa.

Red. Oper. I.

Habitat in Phafiano Motmot.

Corpus medium. Caput magnum, oua. tum, obrufim. Thorax breuifinius. Ābdomen magnum, globofum.

Motra $32-33$. PEDICVLVS capire cordato rufo, cille abdomine albo bafi atteruato.

Pediculus alliventris Scop. Carn. 105 r. Habitat in Mota illa Troglodyte.

Gryl- 33-34. PEDICVLVS fanguineus, pedibus albis.

Pediculus rofratus Scop. Carn. ros2. Habitat in Acheta Gryilotalpa.

Roftrum elongatum, inflexum, nigrum. Corpus fanguineum, immaculatum.

Redta- 3-4. ACARVS obouatus planus: macula witus bafeos obouata. Linn. Syft. Nat. 2. 1022.3. Fn Sv. 1966. It. oel. 62. 126.

Acarus Raj. Inf. 9.

Habitat in Europæ Bobus, Canibus Nimis affinis A. Ricino videtur.

ameo. 7-8. ACARVS obouatus fufcus, lineolis latus duobus maculaque poftica palmata viridi aureis.

Acarus aureolatus Pall. Spicil. Zool. fafc. IX. p. 41. tab. 3. fig. 10.

Habitat in America. 




Bound B

TOWSTEND BOOK BINDERY

Kew Market, $\forall \mathrm{K}$
$703-740-3700$ 
